\title{
Vibration Modelling and the Difference Between Different Vibration Models for a Fewer-DOF Parallel Robot
}

\section{Shuai Fan ( $\sim$ fansuai12345@163.com )}

Chengdu University of Technology https://orcid.org/0000-0002-1227-5154

\section{Shouwen Fan}

University of electronic science and technology of china

\section{Xin Zhang}

Baoji University of Arts and Sciences

\section{Guangkui Song}

university of electronic science and technology of china

\section{Weibin Lan}

university of electronic science and technology of china

\section{Original Article}

Keywords: vibration modelling, drilling parallel robot, dynamics, joint clearances, passive branch

Posted Date: July 9th, 2020

DOI: https://doi.org/10.21203/rs.3.rs-40683/v1

License: (c) (1) This work is licensed under a Creative Commons Attribution 4.0 International License. Read Full License 


\title{
Vibration modelling and the difference between different vibration models for a fewer-DOF parallel robot
} Shuai Fan ${ }^{\mathrm{a}, \text { b* }}$, Shouwen Fan ${ }^{\mathrm{c}}$, Xin Zhang ${ }^{\mathrm{d}}$, Guangkui Song ${ }^{\mathrm{b}}$, Weibin Lan ${ }^{\mathrm{c}}$

${ }^{a}$ The College of Nuclear Technology and Automation Engineering, Chengdu University of Technology, Chengdu, Sichuan, 610059, People's Republic of China

${ }^{b}$ Center for Robotics, University of Electronic Science and Technology of China, Chengdu, Sichuan, 611731, People's Republic of China

${ }^{c}$ School of Mechanical and Electrical Engineering, University of Electronic Science and Technology of China, Chengdu, Sichuan, 611731, People's Republic of China

${ }^{d}$ College of Mechanical Engineering, Baoji University of Arts and Sciences, Baoji, Shaanxi, 721016, People'sRepublic of China

\begin{abstract}
The accuracy of the vibration model used in the design process directly affects the vibration performance of a parallel robot in practice, which determines the machining accuracy and the surface finish of the manufactured products. Considering a drilling parallel robot with a passive branch and few degrees of freedom as the implementation object, a vibration modelling method is proposed in which Kane's equation is utilized, and various commonly ignored factors, such as the passive branch, the joint clearances and gravity, are considered. To explore the effects of the passive branch, which was considered ideal in previous studies, two dynamic models are derived in which the passive branch is rigid or flexible. To explore the effects of the joint clearances, which were ignored in previous studies, two stiffness models of branches are derived, in which the joint clearances are considered or ignored. Finally, numerical examples are presented for analysing the effects of these commonly ignored factors on the vibration performance of the drilling parallel robot. Regarding to the effects of these commonly ignored factors, the findings of this paper can serve as a reference for designers in simplifying the vibration model in the design process of parallel robot.
\end{abstract}

Keywords: vibration modelling, drilling parallel robot, dynamics, joint clearances, passive branch.

\section{Introduction}

In the production of aircraft parts, very large numbers of holes must be drilled in various directions on an irregular surface. Since the conventional drilling machine tool cannot satisfy this requirement, many drilling operations on irregular surfaces are artificial; however, the efficiency is low and the processing quality is strongly affected by human factors. Hence, a drilling parallel robot with high stiffness that can machine on irregular surfaces was designed; it is shown in Fig. 1. However, the vibrational performance of the prototype was worse than expected, and the quality of the produced holes in the experiment did not reach the desired level. One of the most important reasons is that the vibration model employed by the drilling parallel robot, which plays an important role in the quality of the produced holes, is too idealized in the design process. Vibration is caused by various complicated factors, including the machine-tool structure, the tool material and shape, the workpiece material, and the working conditions $[1,2]$. According to our prototype test, although the quality of the produced hole can be effectively improved by reducing the feed rate and by changing the material of the workpiece, this approach increases the cost and limits the application scope. These results do not satisfy the original objective of our design; hence, a detailed vibration analysis of the proposed parallel robot is required for identifying the defects of the used vibration model. Thus, the main objective of this paper is to evaluate the accuracy of the vibration model for the drilling parallel robot; then, improvement and optimization can be achieved.

The vibration analysis of parallel robots is complicated due to the highly nonlinear dynamics and multiple degrees of freedom (DOFs) of the vibration model. Despite this, scholars have obtained excellent insights into this field and their research mainly focuses on four aspects: the construction of the vibration model [3-5], the study of the control strategy [6,7], the optimization of the vibrational performance [8-10] and the parametric design [11, 12]. As a research hotspot of vibration analysis since the beginning, the vibration analysis of the Stewart platform has been successful and its studies have gradually transitioned from theoretical vibration analysis to a wide range of practical applications $[13,14]$. Since both the number of DOFs and the number of branches of the Stewart platform are equal

\footnotetext{
${ }^{*}$ Corresponding author.

E-mail address: fansuai12345@163.com (S. Fan);
} 
to six, its Jacobian matrix, stiffness matrix and damping matrix are sixth-order matrices and since the six branches are identical and only the axis-stiffness is considered, the derivation of the vibration equation of the 6-DOF vibration system is feasible. However, when the parallel mechanism is used as a machine tool, the parallel robot with fewer DOFs is more popular due to the associated cost savings and simple control [15-19]; however, relatively few vibration studies have been conducted on parallel robots with fewer DOFs.

The vibration analysis of fewer-DOF parallel robots differs from that of the Stewart platform and two main problems are encountered: the Jacobian matrix is not a square matrix and the stiffness of the branches is not axial [20-23]. To consider the effects of the stiffness along or around non-axial directions of branches and to avoid the need to invert the non-square Jacobian matrix, the energy method was mainly used in previous vibration analyses of parallel robots with fewer DOFs [24, 25]. However, for the energy method, the numbers of elastic nodes and variables are huge and the complexity of the derivation process is high [26,27]. Since Kane's method combines the characteristics of vector mechanics and analytical mechanics, it is especially suitable for non-holonomic systems. Therefore, in this paper, a vibration modelling method is proposed that is based on Kane's method that avoids these drawbacks.

In addition, some fewer-DOF parallel robots have a passive branch $[17,28]$; however, in most studies they are considered rigid bodies so that the number of vibration system DOFs and the number of driving elements are equal $[25,29,30]$. Since the stiffness of the passive branch is neglected, the moving platform will have no vibration in the constrained directions. However, in our evaluation of the prototype, the vibration behaviours in the constrained directions are readily observed and their influence cannot be ignored; therefore, this method is not suitable for applications in high-precision systems. For these reasons, an overall vibration analysis of fewer-DOF parallel robots with passive branches is urgently needed, which is one of the objectives of this paper.

Moreover, the traditional vibration model of parallel robots can be obtained by replacing the branches with the spring-damper system in the dynamic models [14, 31,32]. However, even if the influence of the passive branch is considered in our test of prototype, the traditional spring-damper system still differs from the actual system since the amplitude of the vibration is much larger than the value that is obtained using the spring-damper system. The neglected joint clearances may be the main cause of this phenomenon [33,34], which are unavoidable in the assembly. Therefore, the effects of the joint clearances on the vibrational performance should also be considered, which is another objective of this paper.

Furthermore, the commonly ignored effects of gravity and damping in traditional vibration models are also considered so that the shortcomings of the used vibration model can be identified. The remainder of this paper is organized as follows: The kinematics and dynamics are presented in Section 2. Next, two vibration systems are presented in Section 3. Then, numerical examples, comparisons, discussions and results are presented in Section 4. Finally, the study's conclusions are discussed in Section 5.

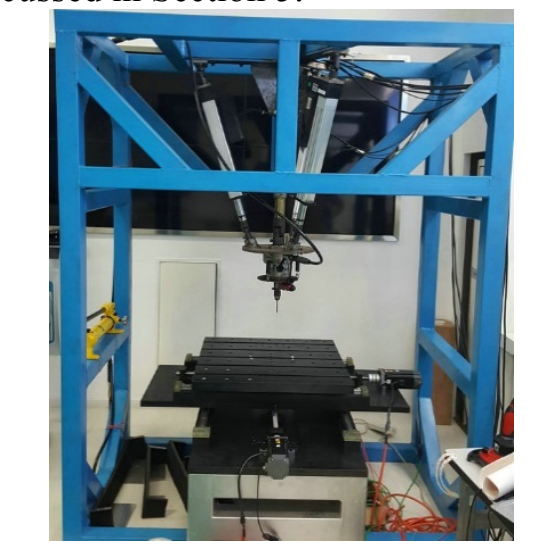

Fig. 1 Prototype of the drilling parallel robot

\section{Kinematics and Dynamics}

In this section, two kinematic analysis models and two dynamic analysis modes of the considered parallel robot are presented. In the first kinematic analysis model, the parallel robot is regarded as a traditional 3-DOF system in 
which the passive branch is rigid. If the stiffness of the passive branch is considered, the mobile platform of the parallel robot can be considered unconstrained in a tiny vibration space. Therefore, in the second kinematic analysis model, the shown parallel robot is regarded as a constrained 6-DOF system, where the passive branch is flexible. According to the screw analysis of the considered drilling parallel robot, one reciprocal wrench of the active branch screw system, which is a pure constraint force along the axes of the branches, and three reciprocal wrenches of the passive branch screw system, of which two are pure constraint forces along the rotation axes of the universal joints and one is a couple around the axis that is perpendicular to the two rotation axes of the universal joint, can be obtained [35]. Therefore, the dynamics analyses of the 3-DOF vibration system and the 6-DOF vibration system are conducted separately based on Kane's method. For the 3-DOF vibration system, only three reciprocal wrenches of active branches are considered and for the 6-DOF vibration system, all reciprocal wrenches are considered. In addition, some matrices that are negligible in the vibration modelling of the 3-DOF and 6-DOF systems are shown in Appendixes A and Appendixes B, respectively.

\subsection{Architecture}

As illustrated in Fig. 2, a 1PU+3UPS parallel robot is selected as the drilling machine tool, where $\mathrm{P}$ denotes a prismatic joint, $\mathrm{U}$ a universal joint, $\mathrm{S}$ a spherical joint, and the underlined letter represents a driving joint. The 1PU+3UPS parallel robot consists of a mobile platform, a fixed platform, three active branches and one passive branch. The three active branches are distributed uniformly on the mobile and fixed platforms and the passive branch is located at the geometric centres of the two platforms. The universal joint of the passive branch is connected to the fixed platform and the universal joints of the three active branches are connected to the mobile platform; hence, the proposed parallel robot has two rotation DOFs and one translation DOF. Since a separate drilling device is mounted on the mobile platform of the parallel robot, which is used to realize the rotation and feed motion of the drill, the proposed parallel robot can drill holes in various directions.

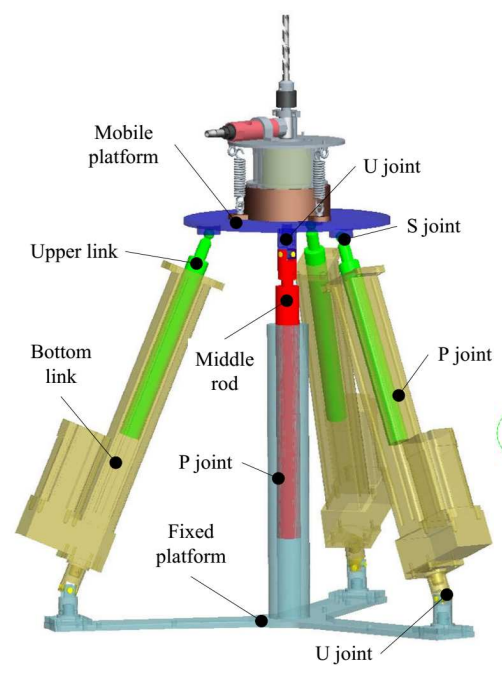

Fig. 2 Three-dimensional model of the drilling parallel robot

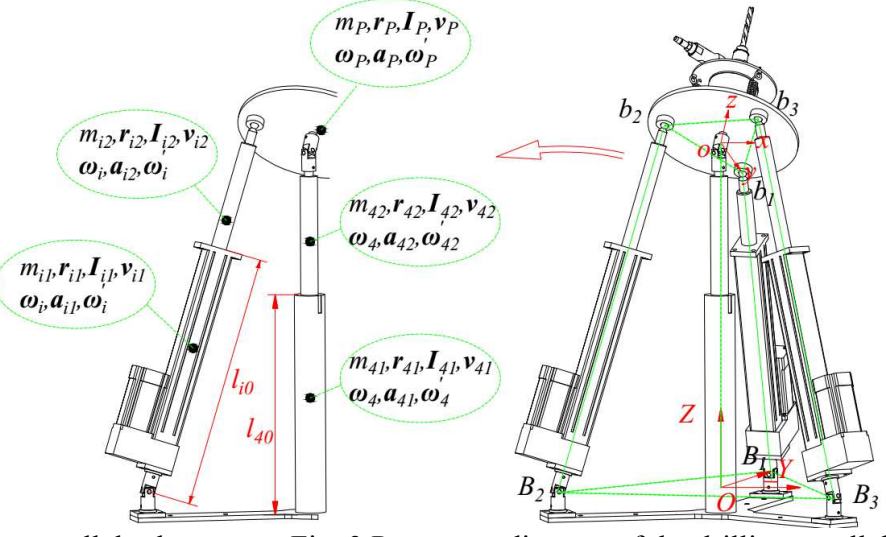

Fig. 3 Parameter diagram of the drilling parallel robot

\subsection{Kinematic constraint equation}

For the kinematic analysis, a reference frame, namely, $O-X Y Z$, is fixed on the fixed platform and another reference frame, namely, $o-x y z$ is attached and followed by the mobile platform, as shown in Fig. 3. The Z-axis and z-axis are perpendicular to the fixed platform and the mobile platform, respectively, and the $Y$-axis and $y$-axis are defined in the directions from the two origins to the two centres of the joints that are connected to the $1^{\text {th }}$ branch, respectively. The two origins of the two reference frames are located at the centres of the two platforms and the $X$-axis and $x$ axis are defined according to the right-hand rule. $\boldsymbol{B}_{i}$ and $\boldsymbol{b}_{i}$ are the centres of the joints that are connected to the fixed and mobile platforms, respectively. The three components of each three-dimensional vector are denoted by symbols 
with the subscripts $x, y$, and $z$, such as $\boldsymbol{B}_{1}=\left(B_{l x}, B_{l y}, B_{l z}\right)$, and these notations are also used in the later discussion. Therefore, the constraint equations that are associated with each branch can be expressed as

$$
l_{i} \boldsymbol{l}_{i}=\boldsymbol{b}_{i}-\boldsymbol{B}_{i} \quad(i=1,2,3),
$$

where $l_{i}$ and $\boldsymbol{l}_{i}$ are the length and unit vector, respectively, of the $i^{\text {th }}$ active branch. In reference frame $O-X Y Z, B i$ can be calculated as the radius of the fixed platform and $\boldsymbol{b}_{i}$ can be calculated as

$$
\boldsymbol{b}_{i}=l_{\mathbf{4}} \boldsymbol{l}_{\mathbf{4}}+\boldsymbol{R} \boldsymbol{b}_{i \mathbf{0}}
$$

where $\boldsymbol{b}_{i 0}$ is the location of the spherical joint in reference frame $o-x y z$ and $\boldsymbol{R}$ is the orientation matrix. Therefore, the length and unit vector of the $i^{\text {th }}$ active branch can be calculated by

$$
l_{i}=\left\|l_{4} \boldsymbol{l}_{\mathbf{4}}+\boldsymbol{R} \boldsymbol{b}_{i \mathbf{0}}-\boldsymbol{B}_{i}\right\|
$$

and

$$
\boldsymbol{l}_{i}=\frac{1}{l_{i}}\left(l_{4} \boldsymbol{l}_{\mathbf{4}}+\boldsymbol{R} \boldsymbol{b}_{i \mathbf{0}}-\boldsymbol{B}_{i}\right),
$$

where $l_{4}$ and $\boldsymbol{l}_{4}$ are the length and unit vector of the passive branch. They can be expressed in terms of the location of the origin of frame $o-x y z$ as

$$
l_{4}=\|\boldsymbol{o}\|, \boldsymbol{l}_{4}=\frac{\boldsymbol{o}}{\|\boldsymbol{o}\|}
$$

\subsection{Kinematics of the 3-DOF system}

If the passive branch and the mobile platform are rigid, the centre of mass of the mobile platform cannot move along the two rotation axes of the universal joint that is connected to the passive branch and cannot rotate around the axis that is perpendicular to the two rotation axes of this universal joint. Consequently, the position and orientation of the centre of mass of the mobile platform can be specified by $\boldsymbol{o}=\left(0,0, l_{4}\right)^{\mathrm{T}}$ and two Euler angles (denoted by $\alpha$ and $\beta$ ), respectively; hence, the coordinates of the mobile platform can be expressed as

$$
\boldsymbol{r}_{p}=\left[\begin{array}{lll}
\alpha & \beta & l_{4}
\end{array}\right]^{T} \text {. }
$$

Differentiating Eq. (6) with respect to time yields

$$
\boldsymbol{r}_{p}^{\prime}=\left[\begin{array}{lll}
\alpha^{\prime} & \beta^{\prime} & l_{4}^{\prime}
\end{array}\right]^{T},
$$

and differentiating Eq. (7) with respect to time yields

$$
\boldsymbol{r}_{p}^{\prime \prime}=\left[\begin{array}{lll}
\alpha^{\prime \prime} & \beta^{\prime \prime} & l_{4}^{\prime \prime}
\end{array}\right]^{T} \text {. }
$$

For the centre of mass of the mobile platform, the linear velocity $\boldsymbol{v}_{p}$, the angular velocity $\boldsymbol{\omega}_{p}$, the linear acceleration $\boldsymbol{a}_{p}$ and the linear acceleration $\boldsymbol{\omega}_{p}^{\prime}$ can be expressed in matrix form as

$$
\boldsymbol{v}_{p}=\boldsymbol{J}_{p} \boldsymbol{r}_{p}^{\prime}, \boldsymbol{\omega}_{p}=\boldsymbol{J}_{\omega p} \boldsymbol{r}_{p}^{\prime}, \boldsymbol{a}_{p}=\boldsymbol{J}_{p} \boldsymbol{r}_{p}^{\prime \prime}, \boldsymbol{\omega}_{p}^{\prime}=\boldsymbol{J}_{\omega p} \boldsymbol{r}_{p}^{\prime \prime},
$$

where

$$
\boldsymbol{J}_{p}=\left[\begin{array}{lll}
0 & 0 & 0 \\
0 & 0 & 0 \\
0 & 0 & 1
\end{array}\right], \boldsymbol{J}_{\omega p}=\left[\begin{array}{lll}
1 & 0 & 0 \\
0 & 1 & 0 \\
0 & 0 & 0
\end{array}\right] .
$$

For the three active branches, the linear and angular velocities of the branches, the linear velocities of the centres of mass of the links, the linear accelerations of the centres of mass of the links and the angular accelerations of branches should be calculated. Differentiating Eq. (1) with respect to time yields

$$
l_{i}^{\prime} \boldsymbol{l}_{i}+l_{i} \boldsymbol{\omega}_{i} \times \boldsymbol{l}_{i}=l_{\mathbf{4}}^{\prime} \boldsymbol{l}_{\mathbf{4}}+l_{\mathbf{4}} \boldsymbol{\omega}_{\mathbf{4}} \times \boldsymbol{l}_{\mathbf{4}}+\boldsymbol{R}^{\prime} \boldsymbol{b}_{i \mathbf{0}} \quad(i=1,2,3),
$$

where

$$
\boldsymbol{R}^{\prime}=\frac{\partial \boldsymbol{R}}{\partial \alpha} \alpha^{\prime}+\frac{\partial \boldsymbol{R}}{\partial \beta} \beta^{\prime}=\boldsymbol{R}_{\alpha}^{\prime} \alpha^{\prime}+\boldsymbol{R}_{\beta}^{\prime} \beta^{\prime},
$$


$\boldsymbol{\omega}_{i}$ is the angular velocity of the $i^{\text {th }}$ active branch, and $\boldsymbol{\omega}_{4}$ is the angular velocity of the passive branch. Since the passive branch is rigid, namely, it can only move along its axial axis and $\boldsymbol{\omega}_{4}=\mathbf{0}$, Eq. (10) can be re-expressed as

$$
l_{i}^{\prime} \boldsymbol{l}_{i}+l_{i} \boldsymbol{\omega}_{i} \times \boldsymbol{l}_{i}=l_{\mathbf{4}}^{\prime} \boldsymbol{l}_{\mathbf{4}}+\boldsymbol{R}^{\prime} \boldsymbol{b}_{i \mathbf{0}}
$$

Taking the dot product with $\boldsymbol{l}_{i}$ on both sides of Eq. (11) and imposing the condition $\boldsymbol{l}_{i}^{T} \boldsymbol{\omega}_{i}=\mathbf{0}$ yields

$$
l_{i}^{\prime}=\boldsymbol{l}_{i}^{T}\left(l_{\mathbf{4}}^{\prime} \boldsymbol{l}_{\mathbf{4}}+\boldsymbol{R}^{\prime} \boldsymbol{b}_{i \mathbf{0}}\right)
$$

According to Eq. (10), the linear velocity of the $i^{\text {th }}$ active branch can be expressed as

$$
\boldsymbol{v}_{i}=l_{4}^{\prime} \boldsymbol{l}_{\mathbf{4}}+\boldsymbol{R}^{\prime} \boldsymbol{b}_{i \mathbf{0}}
$$

Substituting Eq. (12) into Eq. (13), the linear velocity of the $i^{\text {th }}$ active branch can be obtained as

$$
\boldsymbol{v}_{i}=\boldsymbol{J}_{i} \boldsymbol{r}_{p}^{\prime}
$$

where

$$
\boldsymbol{J}_{i}=\left[\boldsymbol{R}_{\alpha}^{\prime} \boldsymbol{b}_{i 0}, \boldsymbol{R}_{\beta}^{\prime} \boldsymbol{b}_{i 0}, \boldsymbol{l}_{\mathbf{4}}\right]
$$

Taking the cross product with $\boldsymbol{l}_{i}$ on both sides of Eq. (11) yields

$$
\boldsymbol{l}_{i} \times\left(\boldsymbol{\omega}_{i} \times \boldsymbol{l}_{i}\right)=\frac{\mathbf{1}}{l_{i}}\left(l_{\mathbf{4}}^{\prime} \boldsymbol{l}_{i} \times \boldsymbol{l}_{\mathbf{4}}+\boldsymbol{l}_{i} \times \boldsymbol{R}^{\prime} \boldsymbol{b}_{i \mathbf{0}}\right) .
$$

Since $\boldsymbol{l}_{i}^{T} \boldsymbol{\omega}_{i}=\mathbf{0}, \boldsymbol{l}_{i} \times\left(\boldsymbol{l}_{i} \times \boldsymbol{\omega}_{i}\right)=\boldsymbol{\omega}_{i}$, the angular velocity of the $i^{\text {th }}$ active branch can be expressed as

$$
\boldsymbol{\omega}_{i}=\boldsymbol{J}_{\omega i} \boldsymbol{r}_{p}^{\prime},
$$

where

$$
\boldsymbol{J}_{\omega i}=\frac{1}{l_{i}}\left[\begin{array}{lll}
\boldsymbol{l}_{i} \times \boldsymbol{R}_{\alpha}^{\prime} \boldsymbol{b}_{i 0} & \boldsymbol{l}_{i} \times \boldsymbol{R}_{\beta}^{\prime} \boldsymbol{b}_{i \mathbf{0}} & \boldsymbol{l}_{i} \times \boldsymbol{l}_{\mathbf{4}}
\end{array}\right]
$$

Considering the influence of inertial forces, the angular acceleration of each branch should be determined. Differentiating Eq. (16) with respect to time, the angular acceleration of the $i^{\text {th }}$ active branch can be obtained as

$$
\boldsymbol{\omega}_{i}^{\prime}=\boldsymbol{J}_{\omega i}^{\prime} \boldsymbol{r}_{p}^{\prime}+\boldsymbol{J}_{\omega i} \boldsymbol{r}_{p}^{\prime \prime}
$$

For the bottom link of the $i^{\text {th }}$ active branch, the location of the centre of mass can be expressed as

$$
\boldsymbol{r}_{i \mathbf{1}}=\boldsymbol{B}_{i}+\frac{l_{i \mathbf{0}}}{\mathbf{2}} \boldsymbol{l}_{i}
$$

where $l_{i o}$ is the length of the bottom link. Differentiating Eq. (18) with respect to time yields the linear velocity as

$$
\boldsymbol{v}_{i \mathbf{1}}=\boldsymbol{r}_{i \mathbf{1}}^{\prime}=\frac{l_{i \mathbf{0}}}{\mathbf{2}} \boldsymbol{\omega}_{i} \times \boldsymbol{l}_{i}
$$

Taking the cross product with $\boldsymbol{l}_{i}$ on both sides of Eq. (15) yields

$$
\boldsymbol{\omega}_{i} \times \boldsymbol{l}_{i}=\frac{\mathbf{1}}{l_{i}}\left(l_{\mathbf{4}}^{\prime} \boldsymbol{l}_{i} \times \boldsymbol{l}_{\mathbf{4}}+\boldsymbol{l}_{i} \times \boldsymbol{R}^{\prime} \boldsymbol{b}_{i \mathbf{0}}\right) \times \boldsymbol{l}_{i}=\frac{\mathbf{1}}{l_{i}}\left(\boldsymbol{E}-\boldsymbol{l}_{i} \boldsymbol{l}_{i}^{T}\right)\left(l_{4}^{\prime} \boldsymbol{l}_{\mathbf{4}}+\boldsymbol{R}^{\prime} \boldsymbol{b}_{i \mathbf{0}}\right) .
$$

where $\boldsymbol{E}$ is a $3^{\text {rd }}$-order unit matrix. Substituting Eq. (20) into Eq. (19), the linear velocity of the centre of mass of the bottom link of the $i^{\text {th }}$ active branch can be solved as

$$
\boldsymbol{v}_{i 1}=\boldsymbol{J}_{i 1} \boldsymbol{r}_{p}^{\prime} \text {, }
$$

where

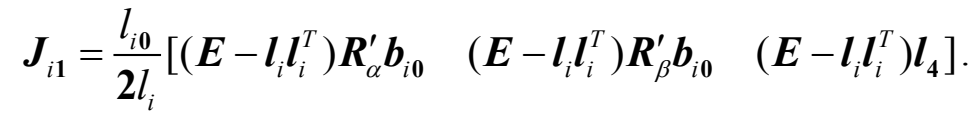

Differentiating Eq. (22) with respect to time yields

$$
\boldsymbol{a}_{i 1}=\boldsymbol{J}_{i 1}^{\prime} \boldsymbol{r}_{p}^{\prime}+\boldsymbol{J}_{i 1} \boldsymbol{r}_{p}^{\prime \prime}
$$

For the upper link of the $i^{\text {th }}$ active branch, the location of the centre of mass can be expressed as 


$$
\boldsymbol{r}_{i 2}=\boldsymbol{B}_{i}+\frac{l_{i}+l_{i 0}}{\mathbf{2}} \boldsymbol{l}_{i}
$$

Differentiating Eq. (23) with respect to time yields

$$
\boldsymbol{v}_{i 2}=\boldsymbol{r}_{i 2}^{\prime}=\frac{l_{i}^{\prime}}{\mathbf{2}} \boldsymbol{l}_{i}+\frac{l_{i}+l_{i 0}}{\mathbf{2}} \boldsymbol{\omega}_{i} \times \boldsymbol{l}_{i}
$$

Substituting Eq. (20) into Eq. (24), the linear velocity of the centre of mass of the upper link of the $i^{\text {th }}$ active branch can be calculated as

$$
\boldsymbol{v}_{i 2}=\boldsymbol{J}_{i 2} \boldsymbol{r}_{p}^{\prime} \text {, }
$$

where

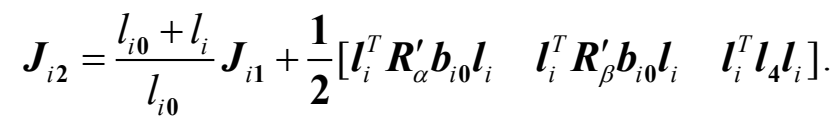

Differentiating Eq. (25) with respect to time yields

$$
\boldsymbol{a}_{i 2}=\boldsymbol{J}_{i 2}^{\prime} \boldsymbol{r}_{p}^{\prime}+\boldsymbol{J}_{i 2} \boldsymbol{r}_{p}^{\prime \prime} \text {, }
$$

For the passive branch, the location of the centre of mass of the upper link can be expressed as

$$
\boldsymbol{r}_{42}=\frac{l_{4}+l_{40}}{2} \boldsymbol{l}_{4}
$$

where $l_{40}$ is the length of the bottom link of the passive branch. By differentiating with respect to time, it is easy to obtain the velocity and acceleration as

$$
\boldsymbol{v}_{42}=\boldsymbol{J}_{42} \boldsymbol{r}_{p}^{\prime} \text {, }
$$

and

$$
\boldsymbol{a}_{42}=\boldsymbol{J}_{42} \boldsymbol{r}_{p}^{\prime \prime}
$$

where

$$
J_{42}=\left[0,0, \frac{l_{4}}{2}\right]
$$

Moreover, by rearranging Eq. (12) in matrix form, the Jacobian matrix of the parallel robot can be determined as

$$
\boldsymbol{l}^{\prime}=\boldsymbol{J} \boldsymbol{r}_{p}^{\prime},
$$

where

$$
\boldsymbol{l}^{\prime}=\left[\begin{array}{lll}
l_{1}^{\prime} & l_{2}^{\prime} & l_{3}^{\prime}
\end{array}\right]^{T}, \quad \boldsymbol{J}=\left[\begin{array}{lll}
\boldsymbol{l}_{\mathbf{1}}^{T} \boldsymbol{R}_{\alpha}^{\prime} \boldsymbol{b}_{\mathbf{1 0}} & \boldsymbol{l}_{\mathbf{1}}^{T} \boldsymbol{R}_{\beta}^{\prime} \boldsymbol{b}_{\mathbf{1 0}} & \boldsymbol{l}_{\mathbf{1}}^{T} \boldsymbol{l}_{\mathbf{4}} \\
\boldsymbol{l}_{\mathbf{2}}^{T} \boldsymbol{R}_{\alpha}^{\prime} \boldsymbol{b}_{\mathbf{2 0}} & \boldsymbol{l}_{\mathbf{2}}^{T} \boldsymbol{R}_{\beta}^{\prime} \boldsymbol{b}_{\mathbf{2 0}} & \boldsymbol{l}_{\mathbf{2}}^{T} \boldsymbol{l}_{\mathbf{4}} \\
\boldsymbol{l}_{\mathbf{3}}^{T} \boldsymbol{R}_{\alpha}^{\prime} \boldsymbol{b}_{\mathbf{3 0}} & \boldsymbol{l}_{\mathbf{3}}^{T} \boldsymbol{R}_{\beta}^{\prime} \boldsymbol{b}_{\mathbf{3 0}} & \boldsymbol{l}_{\mathbf{3}}^{T} \boldsymbol{l}_{\mathbf{4}}
\end{array}\right]
$$

\subsection{Kinematics of the 6-DOF system}

When the driving elements are locked, the position and orientation of the robot should be unchanged. However, under the action of an external force, the parallel robot will have six DOFs in a tiny workspace due to elastic deformation of the rod. Under this scenario, in the force analysis [35], the parallel robot can be regarded as a robot with six driving elements, of which three are along the axial directions of the three active branches, one is along the $Y$-axis direction, one is along the $X$-axis direction, and one is around the $Z$-axis direction. Therefore, the position and orientation of the centre of mass of the mobile platform can be represented by $\boldsymbol{o}=\left(o_{x}, o_{y}, l_{4}+\rho\right)^{\mathrm{T}}$ and three Euler angles $(\alpha+\theta, \beta+\varphi$, and $\psi)$, respectively. The three parasitic motions $\left(o_{x}, o_{y}\right.$ and $\left.\psi\right)$ are much smaller than the three original motions $\left(\alpha, \beta\right.$ and $\left.l_{4}\right)$ of the 3-DOF system and $\theta, \psi$ and $\rho$ have the same physical meaning as the three original motions but are much smaller. If the position and orientation of the mobile platform are specified, then the three original motions are constant, the motions of the mobile platform can be expressed in a tiny vibration space as

$$
\boldsymbol{r}_{p}=\left[\theta, \varphi, \psi, o_{x}, o_{y}, \rho\right]^{T}
$$


Differentiating Eq. (32) with respect to time yields

$$
\boldsymbol{r}_{p}^{\prime}=\left[\theta^{\prime}, \varphi^{\prime}, \psi^{\prime}, o_{x}^{\prime}, o_{y}^{\prime}, \rho^{\prime}\right]^{T},
$$

and differentiating Eq. (34) with respect to time yields

$$
\boldsymbol{r}_{p}^{\prime \prime}=\left[\theta^{\prime \prime}, \varphi^{\prime \prime}, \psi^{\prime \prime}, o_{x}^{\prime \prime}, o_{y}^{\prime \prime}, \rho^{\prime \prime}\right]^{T},
$$

The velocity and linear acceleration of the centre of mass of the mobile platform can also be expressed as

$$
\boldsymbol{v}_{p}=\boldsymbol{J}_{p} \boldsymbol{r}_{p}^{\prime}, \boldsymbol{\omega}_{p}=\boldsymbol{J}_{\omega p} \boldsymbol{r}_{p}^{\prime}, \boldsymbol{a}_{p}=\boldsymbol{J}_{p} \boldsymbol{r}_{p}^{\prime \prime}, \boldsymbol{\omega}_{p}^{\prime}=\boldsymbol{J}_{\omega p} \boldsymbol{r}_{p}^{\prime \prime},
$$

where

$$
\boldsymbol{J}_{p}=\left[\begin{array}{ll}
\boldsymbol{0}_{3 \times 3} & \boldsymbol{E}_{\mathbf{3} \times \mathbf{3}}
\end{array}\right] \text {, and } \boldsymbol{J}_{\omega p}=\left[\begin{array}{ll}
\boldsymbol{E}_{\mathbf{3} \times \mathbf{3}} & \boldsymbol{0}_{3 \times \mathbf{3}}
\end{array}\right]
$$

For the three active branches, differentiating Eq. (1) with respect to time yields

$$
l_{i}^{\prime} \boldsymbol{l}_{i}+l_{i} \boldsymbol{\omega}_{i} \times \boldsymbol{l}_{i}=\boldsymbol{o}^{\prime}+\boldsymbol{R}^{\prime} \boldsymbol{b}_{i \mathbf{0}} \quad(i=1,2,3),
$$

where

$$
\boldsymbol{R}^{\prime}=\frac{\partial \boldsymbol{R}}{\partial \theta} \theta^{\prime}+\frac{\partial \boldsymbol{R}}{\partial \varphi} \varphi^{\prime}+\frac{\partial \boldsymbol{R}}{\partial \psi} \psi^{\prime}=\boldsymbol{R}_{\theta}^{\prime} \theta^{\prime}+\boldsymbol{R}_{\varphi}^{\prime} \varphi^{\prime}+\boldsymbol{R}_{\psi}^{\prime} \psi^{\prime}
$$

Taking the dot product and cross product with $\boldsymbol{l}_{i}$ on both sides, the linear and angular velocities of the $i^{\text {th }}$ active branch can be expressed as

$$
l_{i}^{\prime}=\boldsymbol{l}_{i}^{T} \boldsymbol{o}^{\prime}+\boldsymbol{l}_{i}^{T} \boldsymbol{R}^{\prime} \boldsymbol{b}_{i \mathbf{0}}
$$

and

$$
\boldsymbol{\omega}_{i}=\frac{\mathbf{1}}{l_{i}}\left(\boldsymbol{l}_{i} \times \boldsymbol{o}^{\prime}+\boldsymbol{l}_{i} \times \boldsymbol{R}^{\prime} \boldsymbol{b}_{i \mathbf{0}}\right) .
$$

Consequently, the mapping matrices of the active branches can be expressed as

$$
\boldsymbol{v}_{i}=\boldsymbol{J}_{i} \boldsymbol{r}_{p}^{\prime} \text {, }
$$

and

$$
\boldsymbol{\omega}_{i}=\boldsymbol{J}_{i} \boldsymbol{r}_{p}^{\prime},
$$

where

$$
\begin{aligned}
& \boldsymbol{J}_{i}=\left[\begin{array}{llllll}
\boldsymbol{R}_{\theta}^{\prime} \boldsymbol{b}_{i \mathbf{0}} & \boldsymbol{R}_{\varphi}^{\prime} \boldsymbol{b}_{i \mathbf{0}} & \boldsymbol{R}_{\psi}^{\prime} \boldsymbol{b}_{i \mathbf{0}} & \boldsymbol{n}_{x} & \boldsymbol{n}_{y} & \boldsymbol{n}_{z}
\end{array}\right] \\
& \boldsymbol{J}_{\omega i}=\frac{1}{l_{i}}\left[\begin{array}{llllll}
\boldsymbol{l}_{i} \times \boldsymbol{R}_{\theta}^{\prime} \boldsymbol{b}_{i \mathbf{0}} & \boldsymbol{l}_{i} \times \boldsymbol{R}_{\varphi}^{\prime} \boldsymbol{b}_{i \mathbf{0}} & \boldsymbol{l}_{i} \times \boldsymbol{R}_{\psi}^{\prime} \boldsymbol{b}_{i \mathbf{0}} & \boldsymbol{l}_{i} \times \boldsymbol{n}_{x} & \boldsymbol{l}_{i} \times \boldsymbol{n}_{y} & \boldsymbol{l}_{i} \times \boldsymbol{n}_{z}
\end{array}\right], \\
& \boldsymbol{n}_{x}=[1,0,0]^{T}, \boldsymbol{n}_{y}=[0,1,0]^{T}, \boldsymbol{n}_{z}=[0,0,1]^{T} .
\end{aligned}
$$

Then, utilizing the same procedure as in Section 2.2, the angular acceleration mapping matrices can be obtained. After the angular velocities of the three active branches have been calculated, it is easy to obtain the linear velocities and accelerations of the centres of mass of all links of the three active branches, which can be formulated as

$$
\boldsymbol{v}_{i 1}=\boldsymbol{J}_{i 1} \boldsymbol{r}_{p}^{\prime}, \boldsymbol{v}_{i 2}=\boldsymbol{J}_{i 2} \boldsymbol{r}_{p}^{\prime}, \boldsymbol{a}_{i \mathbf{1}}=\boldsymbol{J}_{i 1}^{\prime} \boldsymbol{r}_{p}^{\prime}+\boldsymbol{J}_{i 1} \boldsymbol{r}_{p}^{\prime \prime}, \boldsymbol{a}_{i 2}=\boldsymbol{J}_{i 2}^{\prime} \boldsymbol{r}_{p}^{\prime}+\boldsymbol{J}_{i 2} \boldsymbol{r}_{p}^{\prime \prime},
$$

where

$$
\boldsymbol{J}_{i \mathbf{1}}=\frac{l_{i \mathbf{0}}}{\boldsymbol{2} l_{i}}\left[\begin{array}{c}
\left(\boldsymbol{E}-\boldsymbol{l}_{i} \boldsymbol{l}_{i}^{T}\right) \boldsymbol{R}_{\theta}^{\prime} \boldsymbol{b}_{i \mathbf{0}} \\
\left(\boldsymbol{E}-\boldsymbol{l}_{i} \boldsymbol{l}_{i}^{T}\right) \boldsymbol{R}_{\varphi}^{\prime} \boldsymbol{b}_{i \mathbf{0}} \\
\left(\boldsymbol{E}-\boldsymbol{l}_{i} \boldsymbol{l}_{i}^{T}\right) \boldsymbol{R}_{\psi}^{\prime} \boldsymbol{b}_{i \mathbf{0}} \\
\left(\boldsymbol{E}-\boldsymbol{l}_{i} \boldsymbol{l}_{i}^{T}\right) \boldsymbol{n}_{x} \\
\left(\boldsymbol{E}-\boldsymbol{l}_{i} \boldsymbol{l}_{i}^{T}\right) \boldsymbol{n}_{y} \\
\left(\boldsymbol{E}-\boldsymbol{l}_{i} \boldsymbol{l}_{i}^{T}\right) \boldsymbol{n}_{z}
\end{array}\right]^{T}, \boldsymbol{J}_{i \mathbf{2}}=\frac{l_{i \mathbf{0}}+l_{i}}{l_{i \mathbf{0}}} \boldsymbol{J}_{i \mathbf{1}}+\frac{\mathbf{1}}{\mathbf{2}}\left[\begin{array}{c}
\boldsymbol{l}_{i}^{T} \boldsymbol{R}_{\theta}^{\prime} \boldsymbol{b}_{i 0} \boldsymbol{l}_{i} \\
\boldsymbol{l}_{i}^{T} \boldsymbol{R}_{\varphi}^{\prime} \boldsymbol{b}_{i 0} \boldsymbol{l}_{i} \\
\boldsymbol{l}_{i}^{T} \boldsymbol{R}_{\psi}^{\prime} \boldsymbol{b}_{i \mathbf{0}} \boldsymbol{l}_{i} \\
\boldsymbol{l}_{i}^{T} \boldsymbol{n}_{x} \boldsymbol{l}_{i} \\
\boldsymbol{l}_{i}^{T} \boldsymbol{n}_{y} \boldsymbol{l}_{i} \\
\boldsymbol{l}_{i}^{T} \boldsymbol{n}_{z} \boldsymbol{l}_{i}
\end{array}\right]^{T}
$$

For the passive branch, the constraint equation is 


$$
\boldsymbol{o}=\left[o_{x}, o_{y}, o_{z}\right]^{T}=l_{\mathbf{4}} \boldsymbol{l}_{\mathbf{4}},
$$

To solve for the velocity of the passive branch, differentiating Eq. (41) with respect to time yields

$$
\boldsymbol{v}_{\mathbf{4}}=\boldsymbol{o}^{\prime}=\left[o_{x}^{\prime}, o_{y}^{\prime}, \rho^{\prime}\right]^{T}=l_{\mathbf{4}}^{\prime} \boldsymbol{l}_{\mathbf{4}}+l_{\mathbf{4}} \boldsymbol{\omega}_{\mathbf{4}} \times \boldsymbol{l}_{\mathbf{4}},
$$

Taking the dot product with $\boldsymbol{l}_{4}$ on both sides of Eq. (42) yields

$$
l_{4}^{\prime}=\frac{o_{x}}{l_{4}} o_{x}^{\prime}+\frac{o_{y}}{l_{4}} o_{y}^{\prime}+\frac{o_{z}}{l_{4}} \rho^{\prime}
$$

To determine the angular velocity of the passive branch, taking the cross product with $l_{4}$ on both sides of Eq. (42) yields

$$
\boldsymbol{l}_{4} \times\left[o_{x}^{\prime}, o_{y}^{\prime}, \rho^{\prime}\right]=\boldsymbol{l}_{4} \times l_{4}^{\prime} \boldsymbol{l}_{4}+\boldsymbol{l}_{4} \times\left(l_{4} \omega_{4} \times \boldsymbol{l}_{4}\right)
$$

thus,

$$
\boldsymbol{\omega}_{4}=\frac{1}{l_{4}}\left(\boldsymbol{E}-\boldsymbol{l}_{4} \boldsymbol{l}_{4}^{T}\right)^{-1} \boldsymbol{l}_{\mathbf{4}} \times\left[o_{x}^{\prime}, o_{y}^{\prime}, \rho^{\prime}\right]
$$

Since the passive branch has a parasitic motion around its axis, its angular velocity contains an additional term; hence, the expression for the angular velocity is revised to

$$
\boldsymbol{\omega}_{\mathbf{4}}=\frac{1}{l_{4}}\left(\boldsymbol{E}-\boldsymbol{l}_{\mathbf{4}} \boldsymbol{l}_{\mathbf{4}}^{T}\right)^{-\mathbf{1}} \boldsymbol{l}_{\mathbf{4}} \times\left[o_{x}^{\prime}, o_{y}^{\prime}, \rho^{\prime}\right]+\boldsymbol{n}_{z} \psi^{\prime},
$$

The angular and linear velocities of the passive branch can be expressed in matrix form as

$$
\omega_{4}=J_{\omega \mathbf{4}} \boldsymbol{r}_{p}^{\prime}, \boldsymbol{v}_{\mathbf{4}}=\boldsymbol{J}_{4} \boldsymbol{r}_{p}^{\prime}
$$

where

$$
\begin{aligned}
& \boldsymbol{J}_{\mathbf{4}}=\left[\begin{array}{llllll}
\mathbf{0} & \mathbf{0} & \mathbf{0} & \boldsymbol{n}_{x} & \boldsymbol{n}_{y} & \boldsymbol{n}_{z}
\end{array}\right], \\
& \boldsymbol{J}_{\omega \mathbf{4}}=\left[\begin{array}{llllll}
\mathbf{0} & \mathbf{0} & \boldsymbol{n}_{z} & \frac{1}{l_{\mathbf{4}}}\left(\boldsymbol{E}-\boldsymbol{l}_{\mathbf{4}} \boldsymbol{l}_{\mathbf{4}}^{T}\right)^{-\mathbf{1}} \boldsymbol{l}_{\mathbf{4}} \times \boldsymbol{n}_{x} & \frac{\mathbf{1}}{l_{\mathbf{4}}}\left(\boldsymbol{E}-\boldsymbol{l}_{\mathbf{4}} \boldsymbol{l}_{\mathbf{4}}^{T}\right)^{-\mathbf{1}} \boldsymbol{l}_{\mathbf{4}} \times \boldsymbol{n}_{y} \quad \frac{\mathbf{1}}{l_{\mathbf{4}}}\left(\boldsymbol{E}-\boldsymbol{l}_{\mathbf{4}} \boldsymbol{l}_{\mathbf{4}}^{T}\right)^{-\mathbf{1}} \boldsymbol{l}_{\mathbf{4}} \times \boldsymbol{n}_{z}
\end{array}\right] .
\end{aligned}
$$

In addition, the linear velocity and acceleration of the centre of mass of the links can be expressed as

$$
v_{41}=J_{41} r_{p}^{\prime}, a_{41}=J_{41}^{\prime} r_{p}^{\prime}+J_{41} r_{p}^{\prime \prime}, v_{42}=J_{42} r_{p}^{\prime}, a_{42}=J_{42}^{\prime} r_{p}^{\prime}+J_{42} r_{p}^{\prime \prime}
$$

where

$$
\begin{aligned}
& l_{\mathbf{4}}^{\prime}=\frac{o_{x}}{l_{4}} o_{x}^{\prime}+\frac{o_{y}}{l_{4}} o_{y}^{\prime}+\frac{o_{z}}{l_{4}} \rho^{\prime}, \\
& \boldsymbol{J}_{\mathbf{4 1}}=\frac{l_{\mathbf{4 0}}}{\mathbf{2 l}_{\mathbf{4}}}\left[\begin{array}{llllll}
\mathbf{0} & \mathbf{0} & \mathbf{0} & -\frac{o_{x}}{l_{4}^{2}} \boldsymbol{o}+\boldsymbol{n}_{x} & -\frac{o_{y}}{l_{4}^{2}} \boldsymbol{o}+\boldsymbol{n}_{y} & -\frac{o_{z}}{l_{\mathbf{4}}^{2}} \boldsymbol{o}+\boldsymbol{n}_{z}
\end{array}\right], \\
& \boldsymbol{J}_{\mathbf{4 2}}=\boldsymbol{J}_{\mathbf{4 1}}+\frac{\mathbf{1}}{\mathbf{2}}\left[\begin{array}{llllll}
\mathbf{0} & \mathbf{0} & \mathbf{0} & \boldsymbol{n}_{x} & \boldsymbol{n}_{y} & \boldsymbol{n}_{z}
\end{array}\right],
\end{aligned}
$$

Furthermore, according to Eq. (36), it is easy to calculate the traditional Jacobian matrix of the 6-DOF vibration system as

$$
\boldsymbol{l}^{\prime}=\boldsymbol{J} \boldsymbol{r}_{p}^{\prime} \text {, }
$$

where

$$
\boldsymbol{l}^{\prime}=\left[\begin{array}{lll}
l_{\mathbf{1}}^{\prime} & l_{\mathbf{2}}^{\prime} & l_{\mathbf{3}}^{\prime}
\end{array}\right]^{T}, \quad \boldsymbol{J}=\left[\begin{array}{llllll}
\boldsymbol{l}_{\mathbf{1}}^{T} \boldsymbol{R}_{\theta}^{\prime} \boldsymbol{b}_{\mathbf{1 0}} & \boldsymbol{l}_{\mathbf{1}}^{T} \boldsymbol{R}_{\varphi}^{\prime} \boldsymbol{b}_{\mathbf{1 0}} & \boldsymbol{l}_{\mathbf{1}}^{T} \boldsymbol{R}_{\psi}^{\prime} \boldsymbol{b}_{\mathbf{1 0}} & \boldsymbol{l}_{\mathbf{1}}^{T} \boldsymbol{n}_{x} & \boldsymbol{l}_{\mathbf{1}}^{T} \boldsymbol{n}_{y} & \boldsymbol{l}_{\mathbf{1}}^{T} \boldsymbol{n}_{z} \\
\boldsymbol{l}_{\mathbf{2}}^{T} \boldsymbol{R}_{\theta}^{\prime} \boldsymbol{b}_{\mathbf{2 0}} & \boldsymbol{l}_{\mathbf{2}}^{T} \boldsymbol{R}_{\varphi}^{\prime} \boldsymbol{b}_{\mathbf{2 0}} & \boldsymbol{l}_{\mathbf{2}}^{T} \boldsymbol{R}_{\psi}^{\prime} \boldsymbol{b}_{\mathbf{2 0}} & \boldsymbol{l}_{\mathbf{2}}^{T} \boldsymbol{n}_{x} & \boldsymbol{l}_{\mathbf{2}}^{T} \boldsymbol{n}_{y} & \boldsymbol{l}_{\mathbf{2}}^{T} \boldsymbol{n}_{z} \\
\boldsymbol{l}_{\mathbf{3}}^{T} \boldsymbol{R}_{\theta}^{\prime} \boldsymbol{b}_{\mathbf{3 0}} & \boldsymbol{l}_{\mathbf{3}}^{T} \boldsymbol{R}_{\varphi}^{\prime} \boldsymbol{b}_{\mathbf{3 0}} & \boldsymbol{l}_{\mathbf{3}}^{T} \boldsymbol{R}_{\psi}^{\prime} \boldsymbol{b}_{\mathbf{3 0}} & \boldsymbol{l}_{\mathbf{3}}^{T} \boldsymbol{n}_{x} & \boldsymbol{l}_{\mathbf{3}}^{T} \boldsymbol{n}_{y} & \boldsymbol{l}_{\mathbf{3}}^{T} \boldsymbol{n}_{z}
\end{array}\right] .
$$


The obtained traditional Jacobian matrix is a $3 \times 6$ matrix. To make the Jacobian matrix a square matrix, so that inverse operations can be performed, three virtual drivers are added along the directions of the three parasitic motions. The revised Jacobian matrix can be expressed as

$$
\boldsymbol{l}^{\prime}=\left[\begin{array}{llllll}
l_{\mathbf{1}}^{\prime} & l_{2}^{\prime} & l_{3}^{\prime} & o_{x}^{\prime} & o_{y}^{\prime} & \psi^{\prime}
\end{array}\right]^{T}, \boldsymbol{J}=\left[\begin{array}{ccccccc}
\boldsymbol{l}_{\mathbf{1}}^{T} \boldsymbol{R}_{\theta}^{\prime} \boldsymbol{b}_{\mathbf{1 0}} & \boldsymbol{l}_{\mathbf{1}}^{T} \boldsymbol{R}_{\varphi}^{\prime} \boldsymbol{b}_{\mathbf{1 0}} & \boldsymbol{l}_{\mathbf{1}}^{T} \boldsymbol{R}_{\psi}^{\prime} \boldsymbol{b}_{\mathbf{1 0}} & \boldsymbol{l}_{\mathbf{1}}^{T} \boldsymbol{n}_{x} & \boldsymbol{l}_{\mathbf{1}}^{T} \boldsymbol{n}_{y} & \boldsymbol{l}_{\mathbf{1}}^{T} \boldsymbol{n}_{z} \\
\boldsymbol{l}_{\mathbf{2}}^{T} \boldsymbol{R}_{\theta}^{\prime} \boldsymbol{b}_{\mathbf{2 0}} & \boldsymbol{l}_{\mathbf{2}}^{T} \boldsymbol{R}_{\varphi}^{\prime} \boldsymbol{b}_{\mathbf{2 0}} & \boldsymbol{l}_{\mathbf{2}}^{T} \boldsymbol{R}_{\psi}^{\prime} \boldsymbol{b}_{\mathbf{2 0}} & \boldsymbol{l}_{\mathbf{2}}^{T} \boldsymbol{n}_{x} & \boldsymbol{l}_{\mathbf{2}}^{T} \boldsymbol{n}_{y} & \boldsymbol{l}_{2}^{T} \boldsymbol{n}_{z} \\
\boldsymbol{l}_{3}^{T} \boldsymbol{R}_{\theta}^{\prime} \boldsymbol{b}_{\mathbf{3 0}} & \boldsymbol{l}_{\mathbf{3}}^{T} \boldsymbol{R}_{\varphi}^{\prime} \boldsymbol{b}_{\mathbf{3 0}} & \boldsymbol{l}_{\mathbf{3}}^{T} \boldsymbol{R}_{\psi}^{\prime} \boldsymbol{b}_{\mathbf{3 0}} & \boldsymbol{l}_{\mathbf{3}}^{T} \boldsymbol{n}_{x} & \boldsymbol{l}_{\mathbf{3}}^{T} \boldsymbol{n}_{y} & \boldsymbol{l}_{\mathbf{3}}^{T} \boldsymbol{n}_{z} \\
0 & 0 & 0 & 1 & 0 & 0 \\
0 & 0 & 0 & 0 & 1 & 0 \\
0 & 0 & 1 & 0 & 0 & 0
\end{array}\right]
$$

\subsection{Dynamics of the 3-DOF system}

The core strategy of Kane's method is to use a generalized rate instead of generalized coordinates as an independent variable to describe the motion of the system. For the 3-DOF system, the orientation and position of the centre of the mobile platform can be represented by three parameters, namely, $\alpha, \beta$ and $l_{4}$; hence, the generalized coordinates are selected as

$$
\boldsymbol{q}=\boldsymbol{r}_{p}=\left[\alpha, \beta, l_{4}\right]^{T}=\left[q_{1}, q_{2}, q_{3}\right]^{T} \text {. }
$$

Thus, the generalized velocities are

$$
\boldsymbol{q}^{\prime}=\boldsymbol{r}_{p}^{\prime}=\left[\alpha^{\prime}, \beta^{\prime}, l_{4}^{\prime}\right]^{T}=\left[q_{1}^{\prime}, q_{2}^{\prime}, q_{3}^{\prime}\right]^{T}
$$

Therefore, all velocities of all components can be expressed in terms of the generalized velocities. The partial velocities of all components can be calculated by differentiating the velocities of all parts with respect to the generalized velocities. According to the kinematic analysis of the 3-DOF system that is discussed in Section 2.3, the $f^{\text {th }}$ partial linear velocity and $f^{\text {th }}$ partial angular velocity of the mobile platform can be calculated by

$$
\boldsymbol{v}_{p}^{f}=\frac{\partial \boldsymbol{v}_{p}}{\partial q_{f}^{\prime}}=\left(\boldsymbol{J}_{p}\right)_{c f}, \boldsymbol{\omega}_{p}^{f}=\frac{\partial \boldsymbol{\omega}_{p}}{\partial q_{f}^{\prime}}=\left(\boldsymbol{J}_{\omega p}\right)_{c f} \quad(f=1,2,3),
$$

where $\left(\boldsymbol{J}_{p}\right)_{c f}$ is the $f^{\text {th }}$ column of matrix $\boldsymbol{J}_{p}$ and $\left(\boldsymbol{J}_{\omega p}\right)_{c f}$ is the $f^{\text {th }}$ column of matrix $\boldsymbol{J}_{\omega p}$. Similarly, the $f^{\text {th }}$ partial linear velocity and partial angular velocity of the $i^{\text {th }}$ branch can be calculated as

$$
\boldsymbol{v}_{i}^{f}=\frac{\partial \boldsymbol{v}_{i}}{\partial q_{f}^{\prime}}=\left(\boldsymbol{J}_{i}\right)_{c f}, \boldsymbol{\omega}_{i}^{f}=\frac{\partial \boldsymbol{\omega}_{i}}{\partial q_{f}^{\prime}}=\left(\boldsymbol{J}_{\omega i}\right)_{c f},
$$

the partial linear velocity of the bottom link of the $i^{\text {th }}$ branch can be calculated as

$$
\boldsymbol{v}_{i \mathbf{1}}^{f}=\frac{\partial \boldsymbol{v}_{i \mathbf{1}}}{\partial q_{f}^{\prime}}=\left(\boldsymbol{J}_{i \mathbf{1}}\right)_{c f}
$$

and the partial linear velocity of the upper link of the $i^{\text {th }}$ branch can be calculated as

$$
\boldsymbol{v}_{i 2}^{f}=\frac{\partial \boldsymbol{v}_{i \mathbf{2}}}{\partial q_{f}^{\prime}}=\left(\boldsymbol{J}_{i 2}\right)_{c f} \text {. }
$$

According to Kane's equation, the dynamics equation can be expressed as

$$
\boldsymbol{F}^{(f)}+\boldsymbol{F}^{*(f)}=\mathbf{0}
$$

where $\boldsymbol{F}^{(f)}=\left[F^{(1)}, F^{(2)}, F^{(3)}\right]^{T}$ and $\boldsymbol{F}^{*(f)}=\left[F^{*(1)}, F^{*(2)}, F^{*(3)}\right]^{T}$ are the generalized active forces and the generalized inertial forces, respectively. For the 3-DOF system, the three generalized active forces can be expressed as

$$
F^{(f)}=\sum_{i=1}^{3} \boldsymbol{f}_{i} \cdot \boldsymbol{v}_{i}^{f}+\sum_{i=1}^{3}\left(m_{i 1} \boldsymbol{g} \cdot \boldsymbol{v}_{i \mathbf{1}}^{f}+m_{i 2} \boldsymbol{g} \cdot \boldsymbol{v}_{i 2}^{f}\right)+m_{\mathbf{4 2}} \boldsymbol{g} \cdot \boldsymbol{v}_{\mathbf{4 2}}^{f}+m_{P} \boldsymbol{g} \cdot \boldsymbol{v}_{P}^{f}+\boldsymbol{F}_{e} \cdot \boldsymbol{v}_{P}^{f}+\boldsymbol{T}_{e} \cdot \boldsymbol{\omega}_{P}^{f},
$$

and the three generalized inertial forces can be expressed as 


$$
\begin{aligned}
F^{*(f)}= & -\sum_{i=1}^{3}\left(m_{i 1} \boldsymbol{a}_{i \mathbf{1}} \cdot \boldsymbol{v}_{i \mathbf{1}}^{f}+m_{i 2} \boldsymbol{a}_{i \mathbf{2}} \cdot \boldsymbol{v}_{i \mathbf{2}}^{f}\right)-m_{P} \boldsymbol{a}_{P} \cdot \boldsymbol{v}_{P}^{f}-m_{\mathbf{4 2}} \boldsymbol{a}_{\mathbf{4 2}} \cdot \boldsymbol{v}_{\mathbf{4 2}}^{f}-\left(\boldsymbol{I}_{P} \boldsymbol{\omega}_{P}^{\prime}+\boldsymbol{\omega}_{P} \times \boldsymbol{I}_{P} \boldsymbol{\omega}_{P}\right) \cdot \boldsymbol{\omega}_{P}^{f}- \\
& \sum_{i=1}^{3}\left[\left(\boldsymbol{I}_{i 1} \boldsymbol{\omega}_{i}^{\prime}+\boldsymbol{\omega}_{i} \times \boldsymbol{I}_{i 1} \boldsymbol{\omega}_{i}\right) \cdot \boldsymbol{\omega}_{i \mathbf{1}}^{f}+\left(\boldsymbol{I}_{i 2} \boldsymbol{\omega}_{i}^{\prime}+\boldsymbol{\omega}_{i} \times \boldsymbol{I}_{i 2} \boldsymbol{\omega}_{i}\right) \cdot \boldsymbol{\omega}_{i}^{f}\right]
\end{aligned}
$$

where $\boldsymbol{f}_{1}, \boldsymbol{f}_{2}$ and $\boldsymbol{f}_{3}$ are the reaction forces along the axes of the three active branches. As shown in Fig. $3, m_{i 1}, m_{i 2}$, $m_{41}$ and $m_{P}$ are the masses of the bottom link, the upper link, the rod of the passive branch and the mobile platform, respectively; $\boldsymbol{I}_{i l}, \boldsymbol{I}_{i 2}, \boldsymbol{I}_{4 l}$ and $\boldsymbol{I}_{P}$ are the centroidal body inertia matrices of the corresponding parts; $\boldsymbol{v}_{i l}^{f}, \boldsymbol{v}_{i 2}^{f}, \boldsymbol{v}_{4 l}^{f}$ and $\boldsymbol{v}_{P}^{f}$ are the $f^{\text {th }}$ partial velocities of the corresponding parts; $\boldsymbol{\omega}_{i l}^{f}, \boldsymbol{\omega}_{i 2}^{f}, \boldsymbol{\omega}_{41}^{f}$ and $\boldsymbol{\omega}_{P}^{f}$ are the $f^{\text {th }}$ partial angular velocities of the corresponding parts; $\boldsymbol{v}_{i}^{f}$ is the $f^{\text {th }}$ partial velocity of the $i^{\text {th }}$ branch; $\boldsymbol{F}_{e}$ and $\boldsymbol{T}_{e}$ denote the external load, and $\boldsymbol{g}$ is the gravitational acceleration. Substituting Eqs. (53-56) into Eq. (57) yields

$F^{(f)}=\sum_{i=1}^{\mathbf{3}} \boldsymbol{f}_{i} \cdot\left(\boldsymbol{J}_{i}\right)_{c f}+\sum_{i=1}^{\mathbf{3}}\left(m_{i 1} \boldsymbol{g} \cdot\left(\boldsymbol{J}_{i 1}\right)_{c f}+m_{i 2} \boldsymbol{g} \cdot\left(\boldsymbol{J}_{i 2}\right)_{c f}\right)+m_{\mathbf{4 2}} \boldsymbol{g} \cdot\left(\boldsymbol{J}_{\mathbf{4 2}}\right)_{c f}+m_{P} \boldsymbol{g} \cdot\left(\boldsymbol{J}_{p}\right)_{c f}+\boldsymbol{F}_{e} \cdot\left(\boldsymbol{J}_{i}\right)_{c p}+\boldsymbol{T}_{e} \cdot\left(\boldsymbol{J}_{\omega p}\right)_{c f}$

Substituting all partial velocities and the expressions of all velocities and accelerations into Eq. (59) yields

$$
\begin{aligned}
F^{*(f)}= & -m_{P}\left(\boldsymbol{J}_{p}^{\prime} \boldsymbol{r}_{p}^{\prime}+\boldsymbol{J}_{p} \boldsymbol{r}_{p}^{\prime \prime}\right) \cdot\left(\boldsymbol{J}_{p}\right)_{c f}-\sum_{i=\mathbf{1}}^{3}\left[m_{i 1}\left(\boldsymbol{J}_{i 1}^{\prime} \boldsymbol{r}_{p}^{\prime}+\boldsymbol{J}_{i 1} \boldsymbol{r}_{p}^{\prime \prime}\right) \cdot\left(\boldsymbol{J}_{i \mathbf{1}}\right)_{c f}+m_{i 2}\left(\boldsymbol{J}_{i 2}^{\prime} \boldsymbol{r}_{p}^{\prime}+\boldsymbol{J}_{i 2} \boldsymbol{r}_{p}^{\prime \prime}\right) \cdot\left(\boldsymbol{J}_{i 2}\right)_{c f}\right] \\
& -m_{\mathbf{4 2}}\left(\boldsymbol{J}_{42}^{\prime} \boldsymbol{r}_{p}^{\prime}+\boldsymbol{J}_{42} \boldsymbol{r}_{p}^{\prime \prime}\right) \cdot\left(\boldsymbol{J}_{42}\right)_{c f}-\left(\boldsymbol{I}_{p}\left(\boldsymbol{J}_{\omega p}^{\prime} \boldsymbol{r}_{p}^{\prime}+\boldsymbol{J}_{\omega p} \boldsymbol{r}_{p}^{\prime \prime}\right)+\boldsymbol{\omega}_{P} \times \boldsymbol{I}_{P} \boldsymbol{\omega}_{P}\right) \cdot\left(\boldsymbol{J}_{\omega p}\right)_{c f} \\
& -\sum_{i=1}^{3}\left[\left(\left(\boldsymbol{I}_{i \mathbf{1}}+\boldsymbol{I}_{i \mathbf{2}}\right) \boldsymbol{J}_{\omega i}^{\prime} \boldsymbol{r}_{p}^{\prime}+\left(\boldsymbol{I}_{i \mathbf{1}}+\boldsymbol{I}_{i 2}\right) \boldsymbol{J}_{\omega i} \boldsymbol{r}_{p}^{\prime \prime}+\boldsymbol{\omega}_{i} \times \boldsymbol{I}_{i 1} \boldsymbol{\omega}_{i}+\boldsymbol{\omega}_{i} \times \boldsymbol{I}_{i 2} \boldsymbol{\omega}_{i}\right) \cdot\left(\boldsymbol{J}_{\omega i}\right)_{c f}\right]
\end{aligned}
$$

According to Eq. (60) and Eq. (61), the dynamics equation of Eq. (57) can be obtained by

$$
\boldsymbol{F}^{(f)}=\sum_{i=\mathbf{1}}^{\mathbf{3}} \boldsymbol{J}_{i}^{T} \boldsymbol{f}_{i}+\sum_{i=1}^{\mathbf{3}}\left(m_{i 1} \boldsymbol{J}_{i 1}^{T} \boldsymbol{g}+m_{i 2} \boldsymbol{J}_{i 2}^{T} \boldsymbol{g}\right)+m_{\mathbf{4 2}} \boldsymbol{J}_{\mathbf{4 2}}^{T} \boldsymbol{g}+m_{P} \boldsymbol{J}_{P}^{T} \boldsymbol{g}+\boldsymbol{J}_{P}^{T} \boldsymbol{F}_{e}+\boldsymbol{J}_{\omega P}^{T} \boldsymbol{T}_{e}
$$

and

$$
\begin{aligned}
\boldsymbol{F}^{*(f)}= & -m_{P} \boldsymbol{J}_{p}^{T}\left(\boldsymbol{J}_{p}^{\prime} \boldsymbol{r}_{p}^{\prime}+\boldsymbol{J}_{p}^{T} \boldsymbol{r}_{p}^{\prime \prime}\right)-\sum_{i=\mathbf{1}}^{\mathbf{3}}\left[m_{i \mathbf{1}} \boldsymbol{J}_{i \mathbf{1}}^{T}\left(\boldsymbol{J}_{i \mathbf{1}}^{\prime} \boldsymbol{r}_{p}^{\prime}+\boldsymbol{J}_{i \mathbf{1}} \boldsymbol{r}_{p}^{\prime \prime}\right)+m_{i \mathbf{2}} \boldsymbol{J}_{i \mathbf{2}}^{T}\left(\boldsymbol{J}_{i 2}^{\prime} \boldsymbol{r}_{p}^{\prime}+\boldsymbol{J}_{i \mathbf{2}} \boldsymbol{r}_{p}^{\prime \prime}\right)\right] \\
& -m_{\mathbf{4 2}} \boldsymbol{J}_{4 \mathbf{2}}^{T}\left(\boldsymbol{J}_{42}^{\prime} \boldsymbol{r}_{p}^{\prime}+\boldsymbol{J}_{42} \boldsymbol{r}_{p}^{\prime \prime}\right)-\boldsymbol{J}_{\omega p}^{T}\left(\boldsymbol{I}_{p} \boldsymbol{J}_{\omega p}^{\prime} \boldsymbol{r}_{p}^{\prime}+\boldsymbol{I}_{p} \boldsymbol{J}_{\omega p} \boldsymbol{r}_{p}^{\prime \prime}+\boldsymbol{\omega}_{P} \times \boldsymbol{I}_{P} \boldsymbol{\omega}_{P}\right) \\
& \left.-\sum_{i=1}^{3}\left[\boldsymbol{J}_{\omega i}^{T}\left(\left(\boldsymbol{I}_{i \mathbf{1}}+\boldsymbol{I}_{i \mathbf{2}}\right) \boldsymbol{J}_{\omega i}^{\prime} \boldsymbol{r}_{p}^{\prime}+\left(\boldsymbol{I}_{i \mathbf{1}}+\boldsymbol{I}_{i \mathbf{2}}\right) \boldsymbol{J}_{\omega i} \boldsymbol{r}_{p}^{\prime \prime}+\boldsymbol{\omega}_{i \mathbf{1}} \times \boldsymbol{I}_{i 1} \boldsymbol{\omega}_{i 1}\right)+\boldsymbol{\omega}_{i 2} \times \boldsymbol{I}_{i \mathbf{2}} \boldsymbol{\omega}_{i \mathbf{2}}\right)\right]
\end{aligned}
$$

As a result, the dynamics model of the 3-DOF system is obtained.

\subsection{Dynamics of the 6-DOF system}

According to the kinematics of the 6-DOF system that is defined in Section 2.4, the generalized coordinated are selected as

$$
\boldsymbol{q}=\boldsymbol{r}_{p}=\left[\theta, \varphi, \psi, o_{x}, o_{y}, \rho\right]^{T}=\left[q_{1}, q_{2}, q_{3}, q_{4}, q_{5}, q_{6}\right]^{T}
$$

Thus, the generalized velocities are

$$
\boldsymbol{q}^{\prime}=\boldsymbol{r}_{p}^{\prime}=\left[\theta^{\prime}, \varphi^{\prime}, \psi^{\prime}, o_{x}^{\prime}, o_{y}^{\prime}, \rho^{\prime}\right]^{T}=\left[q_{1}^{\prime}, q_{2}^{\prime}, q_{3}^{\prime}, q_{4}^{\prime}, q_{5}^{\prime}, q_{6}^{\prime}\right]^{T}
$$

Since all velocities have the same unified form, all velocities of all components can be expressed in terms of the six generalized velocities. As a result, the generalized active forces and generalized inertial forces can be expressed as

$$
\boldsymbol{F}^{(f)}=\sum_{i=\mathbf{1}}^{\mathbf{3}} \boldsymbol{J}_{i}^{T} \boldsymbol{f}_{i}+\boldsymbol{J}_{\mathbf{4}}^{T} \boldsymbol{f}_{\mathbf{4}}+\boldsymbol{J}_{\mathbf{4}}^{T} \boldsymbol{f}_{\mathbf{5}}+\boldsymbol{J}_{\omega \mathbf{4}}^{T} \boldsymbol{f}_{\mathbf{6}}+\sum_{i=\mathbf{1}}^{4}\left(m_{i 1} \boldsymbol{J}_{i \mathbf{1}}^{T} \boldsymbol{g}+m_{i 2} \boldsymbol{J}_{i 2}^{T} \boldsymbol{g}\right)+m_{P} \boldsymbol{J}_{P}^{T} \boldsymbol{g}+\boldsymbol{J}_{P}^{T} \boldsymbol{F}_{e}+\boldsymbol{J}_{\omega P}^{T} \boldsymbol{T}_{e}
$$




$$
\begin{aligned}
\boldsymbol{F}^{*(f)}= & -\sum_{i=\mathbf{1}}^{4}\left[m_{i 1} \boldsymbol{J}_{i \mathbf{1}}^{T}\left(\boldsymbol{J}_{i \mathbf{1}}^{\prime} \boldsymbol{r}_{p}^{\prime}+\boldsymbol{J}_{i \mathbf{1}} \boldsymbol{r}_{p}^{\prime \prime}\right)+m_{i 2} \boldsymbol{J}_{i \mathbf{2}}^{T}\left(\boldsymbol{J}_{i 2}^{\prime} \boldsymbol{r}_{p}^{\prime}+\boldsymbol{J}_{i 2} \boldsymbol{r}_{p}^{\prime \prime}\right)\right]-\boldsymbol{J}_{\omega p}^{T}\left(\boldsymbol{I}_{p} \boldsymbol{J}_{\omega p}^{\prime} \boldsymbol{r}_{p}^{\prime}+\boldsymbol{I}_{p} \boldsymbol{J}_{\omega p} \boldsymbol{r}_{p}^{\prime \prime}+\boldsymbol{\omega}_{P} \times \boldsymbol{I}_{P} \boldsymbol{\omega}_{P}\right) \\
& \left.-m_{P} \boldsymbol{J}_{p}^{T}\left(\boldsymbol{J}_{p}^{\prime} \boldsymbol{r}_{p}^{\prime}+\boldsymbol{J}_{p} \boldsymbol{r}_{p}^{\prime \prime}\right)-\sum_{i=1}^{4}\left[\boldsymbol{J}_{\omega i}^{T}\left(\left(\boldsymbol{I}_{i \mathbf{1}}+\boldsymbol{I}_{i 2}\right) \boldsymbol{J}_{\omega i}^{\prime} \boldsymbol{r}_{p}^{\prime}+\left(\boldsymbol{I}_{i \mathbf{1}}+\boldsymbol{I}_{i \mathbf{2}}\right) \boldsymbol{J}_{\omega i} \boldsymbol{r}_{p}^{\prime \prime}+\boldsymbol{\omega}_{i \mathbf{1}} \times \boldsymbol{I}_{i 1} \boldsymbol{\omega}_{i \mathbf{1}}\right)+\boldsymbol{\omega}_{i \mathbf{2}} \times \boldsymbol{I}_{i 2} \boldsymbol{\omega}_{i 2}\right)\right]
\end{aligned}
$$

where $\boldsymbol{f}_{4}, \boldsymbol{f}_{5}$ and $\boldsymbol{f}_{6}$ are the constraints of the passive branch along the $X$-axis, along the $Y$-axis and around the $Z$-axis, respectively; hence, the dynamics model of the 6-DOF system is obtained.

\section{Vibration models}

The considered 1PU+3UPS parallel robot has two rotation DOFs around two rotation axes of the central universal joint and one translation DOF along the axis of the passive branch. If the passive branch is rigid, the stiffnesses of only the three active branches are considered, as shown in Fig. 4; however, if the passive branch is flexible, the stiffnesses of all branches should be considered, as shown in Fig. 5. Therefore, two vibration models are constructed as follows.

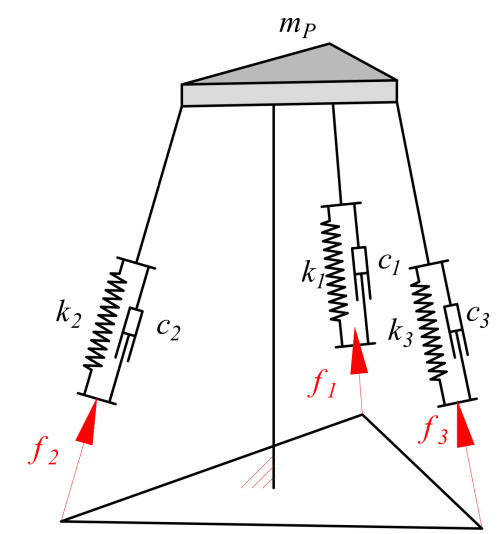

Fig. 4 Structural diagram of the 3-DOF vibration system

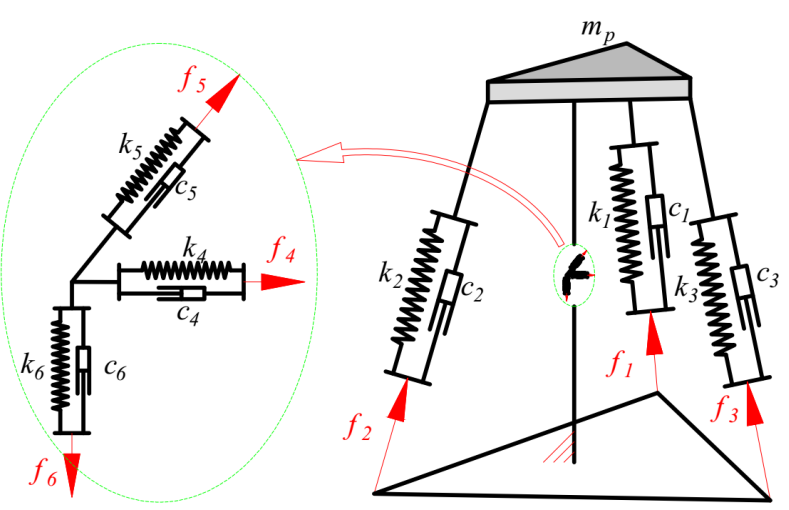

Fig. 5 Structural diagram of the 6-DOF vibration system

\subsection{Constraint of branches}

In previous studies, each branch was considered to be elastic; hence, the elastic deformation was directly proportional to the constraint force, where the scale is called the stiffness coefficient. However, due to the unavoidable joint clearances, the relationship between the constraint force and the elastic deformation is linear and the constant term is not zero but is determined by the joint clearances and the stiffness value. Therefore, two approaches for obtaining the constraint forces of branches for the considered drilling manipulator are presented as follows.

\subsubsection{Ignoring the joint clearances}

Based on the screw analysis and two structural diagrams, one reciprocal wrench of the screw system of the active branch can be obtained, which is a pure constraint force along the axis of the branch. When the three active joints are locked, the constraint force of each active branch at the location of the spherical joint can be expressed as

$$
f_{i}=k_{i} \Delta l_{i}+c_{i} \Delta l_{i}^{\prime} \quad(i=1,2,3) \text {, }
$$

where $k_{i}$ and $c_{i}$ are the equivalent stiffness coefficient and the equivalent damping coefficient of each active branch and $\Delta l_{i}$, and $\Delta l_{i}^{\prime}$ are the displacement and velocity of each active branch. As shown in Fig. 6, each active branch is composed of a universal joint, an electric cylinder (which consists of a bottom link, a ball screw and an upper link), and a spherical joint; hence, the displacement and velocity of the $i^{\text {th }}$ active branch can be obtained via

$$
\frac{1}{k_{i}}=\frac{1}{k_{U i}}+\frac{1}{k_{i 1}}+\frac{1}{k_{P i}}+\frac{1}{k_{i 2}}+\frac{1}{k_{S i}}
$$


and

$$
\frac{\mathbf{1}}{c_{i}}=\frac{\mathbf{1}}{c_{U i}}+\frac{\mathbf{1}}{c_{i 1}}+\frac{\mathbf{1}}{c_{P i}}+\frac{\mathbf{1}}{c_{i 2}}+\frac{\mathbf{1}}{c_{S i}},
$$

where $k_{U i}, k_{i 1}, k_{P i}, k_{i 2}$ and $k_{S i}$ are the stiffnesses of the universal joint, the bottom link, the ball screw, the upper link and the spherical joint, respectively, along the axis direction and $c_{U i}, c_{i l}, c_{P i}, c_{i 2}$ and $c_{S i}$ are the corresponding dampings. Similarly, as shown in Fig. 7, the reactions force of the passive branch can be expressed as

$$
f_{i}=k_{i} \Delta l_{i}+c_{i} \Delta l_{i}^{\prime} \quad(i=4,5,6) \text {, }
$$

where

$$
\frac{1}{k_{i}}=\frac{1}{k_{i 1}}+\frac{1}{k_{P i}}+\frac{1}{k_{i 2}}+\frac{1}{k_{U i}}, \frac{1}{c_{i}}=\frac{1}{c_{i 1}}+\frac{1}{c_{P i}}+\frac{1}{c_{i 2}}+\frac{1}{c_{U i}},
$$

and $k_{i 1}, k_{P i}, k_{i 2}$ and $k_{U i}$ are the stiffnesses of the bottom link, the prismatic joint, the upper link and the universal joint, respectively, and $c_{i 1}, c_{P i}, c_{i 2}$ and $c_{S i}$ are the corresponding dampings. If $i=4$, these parameters are the stiffnesses and dampings of the components along the $X$-axis direction; if $i=5$, these parameters are the stiffnesses and dampings of the components along the $Y$-axis direction; and if $i=6$, these parameters are the stiffnesses and dampings of the components around the $Z$-axis direction.
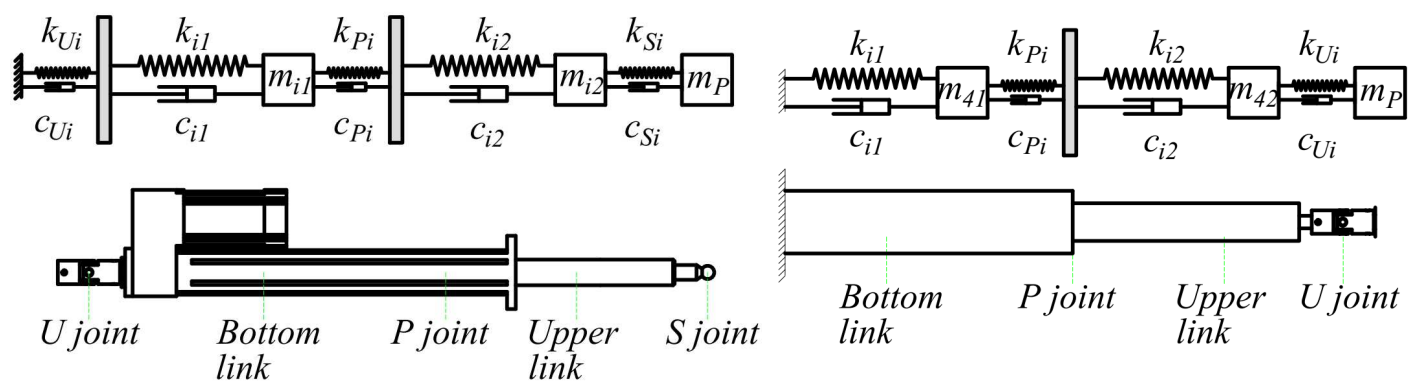

Fig. 6 Stiffness model of the $i^{\text {th }}$ active branch Fig. 7 Stiffness model of the passive branch

\subsubsection{Considering the joint clearances}

The stiffness of a branch depends on four factors: the driver stiffness, the joint clearances, the joint elastic deformations, and the link elastic deformations. For our prototype, the effects of the drive stiffness and the joint elastic deformations are negligible compared with the effects of the joint clearances and the link elastic deformations. Hence, the relationship between the constraints and deformations of the active branches can be expressed as

$$
f_{i}=k_{i}\left(\Delta l_{i}-d_{i}\right)+c_{i} \Delta l_{i}^{\prime} \quad(i=1,2,3)
$$

where

$$
\frac{1}{k_{i}}=\frac{1}{k_{i 1}}+\frac{1}{k_{i 2}}, d_{i}=d_{U i}+d_{P i}+d_{S i}
$$

and $d_{U i}, d_{P i}$ and $d_{S i}$ are the clearances of the universal joint, the prismatic joint and the spherical joint, respectively, along the axis direction. Similarly, the relationship between the constraints and deformations of the passive branches can be expressed as

$$
f_{i}=k_{i}\left(\Delta l_{i}-d_{i}\right)+c_{i} \Delta l_{i}^{\prime} \quad(i=4,5,6),
$$

where

$$
\frac{1}{k_{i}}=\frac{1}{k_{i 1}}+\frac{1}{k_{i 2}}, d_{i}=d_{P i}+d_{U i}
$$

and $d_{P i}$ and $d_{U i}$ are the clearances of the prismatic joint and the spherical joint, respectively. If $i=4$, the stiffnesses and clearances correspond to each component along the $X$-axis direction; if $i=5$, to each component along the $Y$ axis direction; and if $i=6$, to each component around the $Z$-axis direction. 


\subsection{Vibration model of the 3-DOF vibration system}

Substituting the two relationships between the constraints of the active branches into the dynamics model of 3DOF system, two vibration model can be obtained as follows.

\subsubsection{Ignoring the joint clearances}

Considering the directly proportional relationships between the constraints and deformations of the three active branches and assuming that the passive branch is rigid, the generalized active forces that are expressed in Eq. (62) can be re-expressed as

$$
\boldsymbol{F}^{(f)}=-\sum_{i=1}^{3} \boldsymbol{J}_{i}^{T} \boldsymbol{l}_{i}\left(k_{i} \Delta l_{i}+c_{i} \Delta l_{i}^{\prime}\right)+\sum_{i=1}^{3}\left(m_{i 1} \boldsymbol{J}_{i 1}^{T} \boldsymbol{g}+m_{i 2} \boldsymbol{J}_{i 2}^{T} \boldsymbol{g}\right)+m_{\mathbf{4 2}} \boldsymbol{J}_{\mathbf{4 2}}^{T} \boldsymbol{g}+m_{P} \boldsymbol{J}_{P}^{T} \boldsymbol{g}+\boldsymbol{J}_{P}^{T} \boldsymbol{F}_{e}+\boldsymbol{J}_{\omega P}^{T} \boldsymbol{T}_{e}
$$

Then, by substituting Eq. (74) and (67) into Eq. (57), the dynamics model of the 3-DOF vibration system can be obtained as

$$
\boldsymbol{J}_{k}\left[\begin{array}{l}
\Delta l_{1} \\
\Delta l_{2} \\
\Delta l_{3}
\end{array}\right]+\boldsymbol{J}_{c}\left[\begin{array}{l}
\Delta l_{1}^{\prime} \\
\Delta l_{2}^{\prime} \\
\Delta l_{3}^{\prime}
\end{array}\right]-\boldsymbol{G}\left(\boldsymbol{r}_{p}\right) \boldsymbol{g}-\boldsymbol{J}_{p}^{T} \boldsymbol{F}-\boldsymbol{J}_{\omega p}^{T} \boldsymbol{T}+\boldsymbol{M}\left(\boldsymbol{r}_{p}\right) \boldsymbol{r}_{p}^{\prime \prime}+\boldsymbol{M}^{\prime}\left(\boldsymbol{r}_{p}, \boldsymbol{r}_{p}^{\prime}\right) \boldsymbol{r}_{p}^{\prime}=\mathbf{0}
$$

where

$$
\begin{aligned}
& \boldsymbol{J}_{k}=\left[\begin{array}{lll}
k_{\mathbf{1}} \boldsymbol{J}_{\mathbf{1}}^{T} \boldsymbol{l}_{\mathbf{1}} & k_{\mathbf{2}} \boldsymbol{J}_{\mathbf{2}}^{T} \boldsymbol{l}_{\mathbf{2}} & k_{\mathbf{3}} \boldsymbol{J}_{\mathbf{3}}^{T} \boldsymbol{l}_{\mathbf{3}}
\end{array}\right], \\
& \boldsymbol{J}_{c}=\left[\begin{array}{lll}
c_{\mathbf{1}} \boldsymbol{J}_{\mathbf{1}}^{T} \boldsymbol{l}_{\mathbf{1}} & c_{\mathbf{2}} \boldsymbol{J}_{\mathbf{2}}^{T} \boldsymbol{l}_{\mathbf{2}} & c_{\mathbf{3}} \boldsymbol{J}_{\mathbf{3}}^{T} \boldsymbol{l}_{\mathbf{3}}
\end{array}\right], \\
& \boldsymbol{G}\left(\boldsymbol{r}_{p}\right)=\sum_{i=\mathbf{1}}^{\mathbf{3}}\left(m_{i \mathbf{1}} \boldsymbol{J}_{i \mathbf{1}}^{T}+m_{i \mathbf{2}} \boldsymbol{J}_{i \mathbf{2}}^{T}\right)+m_{\mathbf{4 2}} \boldsymbol{J}_{\mathbf{4 2}}^{T}+m_{P} \boldsymbol{J}_{P}^{T}, \\
& \boldsymbol{M}\left(\boldsymbol{r}_{p}\right)=\sum_{i=\mathbf{1}}^{3}\left[m_{i 1} \boldsymbol{J}_{i \mathbf{1}}^{T} \boldsymbol{J}_{i \mathbf{1}}+m_{i \mathbf{2}} \boldsymbol{J}_{i \mathbf{2}}^{T} \boldsymbol{J}_{i \mathbf{2}}+\boldsymbol{J}_{\omega i}^{T}\left(\boldsymbol{I}_{i \mathbf{1}}+\boldsymbol{I}_{i \mathbf{2}}\right) \boldsymbol{J}_{\omega i}\right]+m_{p} \boldsymbol{J}_{p}^{T} \boldsymbol{J}_{p}+\boldsymbol{J}_{\omega p}^{T} \boldsymbol{I}_{p} \boldsymbol{J}_{\omega p}+m_{\mathbf{4 2}} \boldsymbol{J}_{\mathbf{4 2}}^{T} \boldsymbol{J}_{\mathbf{4 2}}, \\
& \boldsymbol{M}^{\prime}\left(\boldsymbol{r}_{p}, \boldsymbol{r}_{p}^{\prime}\right)=\sum_{i=1}^{\mathbf{3}}\left[m_{i \mathbf{1}} \boldsymbol{J}_{i \mathbf{1}}^{T} \boldsymbol{J}_{i \mathbf{1}}^{\prime}+m_{i \mathbf{2}} \boldsymbol{J}_{i 2}^{T} \boldsymbol{J}_{i \mathbf{2}}^{\prime}+\boldsymbol{J}_{\omega i}^{T}\left(\boldsymbol{I}_{i \mathbf{1}}+\boldsymbol{I}_{i \mathbf{2}}\right) \boldsymbol{J}_{\omega i}^{\prime}\right]+m_{p} \boldsymbol{J}_{p}^{T} \boldsymbol{J}_{p}^{\prime}+\boldsymbol{J}_{\omega p}^{T} \boldsymbol{I}_{p} \boldsymbol{J}_{\omega p}^{\prime} .
\end{aligned}
$$

Denoting the tiny deformation of the mobile platform by

$$
\Delta \boldsymbol{P}=\left[\Delta \alpha, \Delta \beta, \Delta l_{4}\right]^{T}
$$

and substituting the Jacobian matrix in Eq. (30) into Eq. (75) yields

$$
\boldsymbol{K}\left(\boldsymbol{r}_{p}\right) \Delta \boldsymbol{P}+\boldsymbol{C}\left(\boldsymbol{r}_{p}\right) \Delta \boldsymbol{P}^{\prime}-\boldsymbol{G}\left(\boldsymbol{r}_{p}\right) \boldsymbol{g}-\boldsymbol{J}_{p}^{T} \boldsymbol{F}-\boldsymbol{J}_{\omega p}^{T} \boldsymbol{T}+\boldsymbol{M}\left(\boldsymbol{r}_{p}\right) \boldsymbol{r}_{p}^{\prime \prime}+\boldsymbol{M}^{\prime}\left(\boldsymbol{r}_{p}, \boldsymbol{r}_{p}^{\prime}\right) \boldsymbol{r}_{p}^{\prime}=\mathbf{0}
$$

where $\boldsymbol{K}\left(\boldsymbol{r}_{p}\right)=\boldsymbol{J}_{k} \boldsymbol{J}$ and $\boldsymbol{C}\left(\boldsymbol{r}_{p}\right)=\boldsymbol{J}_{c} \boldsymbol{J}$. Since the displacement of the vibration is very small, we set $\Delta \boldsymbol{P}^{\prime}=\boldsymbol{r}_{p}^{\prime}$ and $\Delta \boldsymbol{P}^{\prime \prime}=\boldsymbol{r}_{p}^{\prime \prime}$ and the 3-DOF vibration model can be re-expressed as

$$
\boldsymbol{M}\left(\boldsymbol{r}_{p}\right) \Delta \boldsymbol{P}^{\prime \prime}+\left(\boldsymbol{C}\left(\boldsymbol{r}_{p}\right)+\boldsymbol{M}^{\prime}\left(\boldsymbol{r}_{p}, \boldsymbol{r}_{p}^{\prime}\right)\right) \Delta \boldsymbol{P}^{\prime}+\boldsymbol{K}\left(\boldsymbol{r}_{p}\right) \Delta \boldsymbol{P}-\boldsymbol{J}_{p}^{T} \boldsymbol{F}-\boldsymbol{J}_{\omega p}^{T} \boldsymbol{T}-\boldsymbol{G}\left(\boldsymbol{r}_{p}\right) \boldsymbol{g}=\mathbf{0}
$$

\subsubsection{Considering the joint clearances}

Considering the linear relationships between the constraints and the deformations of the three active branches and assuming that the passive branch is rigid, the generalized active forces in Eq. (62) can be expressed as

$$
\boldsymbol{F}^{(f)}=-\sum_{i=1}^{3} \boldsymbol{J}_{i}^{T} \boldsymbol{l}_{i}\left(k_{i} \Delta l_{i}-k_{i} d_{i}+c_{i} \Delta l_{i}^{\prime}\right)+\sum_{i=1}^{4}\left(m_{i 1} \boldsymbol{J}_{i 1}^{T} \boldsymbol{g}+m_{i 2} \boldsymbol{J}_{i 2}^{T} \boldsymbol{g}\right)+m_{P} \boldsymbol{J}_{P}^{T} \boldsymbol{g}+\boldsymbol{J}_{P}^{T} \boldsymbol{F}_{e}+\boldsymbol{J}_{\omega P}^{T} \boldsymbol{T}_{e} .
$$

Substituting Eq. (79) and (63) into Eq. (57), the dynamics model of the 3-DOF vibration model can be re-expressed as 


$$
\boldsymbol{J}_{k}\left[\begin{array}{l}
\Delta l_{1} \\
\Delta l_{\mathbf{2}} \\
\Delta l_{3}
\end{array}\right]-\boldsymbol{J}_{k}\left[\begin{array}{l}
d_{\mathbf{1}} \\
d_{\mathbf{2}} \\
d_{\mathbf{3}}
\end{array}\right]+\boldsymbol{J}_{c}\left[\begin{array}{l}
\Delta l_{1}^{\prime} \\
\Delta l_{2}^{\prime} \\
\Delta l_{3}^{\prime}
\end{array}\right]-\boldsymbol{G}\left(\boldsymbol{r}_{p}\right) \boldsymbol{g}-\boldsymbol{J}_{p}^{T} \boldsymbol{F}-\boldsymbol{J}_{\omega p}^{T} \boldsymbol{T}+\boldsymbol{M}\left(\boldsymbol{r}_{p}\right) \boldsymbol{r}_{p}^{\prime \prime}+\boldsymbol{M}^{\prime}\left(\boldsymbol{r}_{p}, \boldsymbol{r}_{p}^{\prime}\right) \boldsymbol{r}_{p}^{\prime}=\mathbf{0}
$$

Let $\boldsymbol{d}=\left[d_{1}, d_{2}, d_{3}\right]^{\mathrm{T}}$. Then, the 3-DOF vibration model is

$$
\boldsymbol{M}\left(\boldsymbol{r}_{p}\right) \Delta \boldsymbol{P}^{\prime \prime}+\left(\boldsymbol{C}\left(\boldsymbol{r}_{p}\right)-\boldsymbol{M}^{\prime}\left(\boldsymbol{r}_{p}, \boldsymbol{r}_{p}^{\prime}\right)\right) \Delta \boldsymbol{P}^{\prime}+\boldsymbol{K}\left(\boldsymbol{r}_{p}\right) \Delta \boldsymbol{P}-\boldsymbol{J}_{p}^{T} \boldsymbol{F}-\boldsymbol{J}_{\omega p}^{T} \boldsymbol{T}-\boldsymbol{G}\left(\boldsymbol{r}_{p}\right) \boldsymbol{g}-\boldsymbol{J}_{k} \boldsymbol{d}=\mathbf{0}
$$

\subsection{Vibration model of the 6-DOF vibration system}

By substituting the two relationships between the constraints and deformations of all branches into the dynamics model of the 6-DOF system, two vibration models can be obtained as follows.

\subsubsection{Ignoring the joint clearances}

Considering the directly proportional relationships between the constraints and deformations of all branches and assuming that the passive branch is flexible, the generalized active forces of the 6-DOF vibration system that are expressed in Eq. (66) can be re-expressed as

$$
\begin{aligned}
\boldsymbol{F}^{(f)}= & -\sum_{i=\mathbf{1}}^{3} \boldsymbol{J}_{i}^{T} \boldsymbol{l}_{i}\left(k_{i} \Delta l_{i}+c_{i} \Delta l_{i}^{\prime}\right)-\boldsymbol{J}_{\mathbf{4}}^{T} \boldsymbol{n}_{x}\left(k_{\mathbf{4}} \Delta l_{\mathbf{4}}+c_{\mathbf{4}} \Delta l_{\mathbf{4}}^{\prime}\right)-\boldsymbol{J}_{\mathbf{4}}^{T} \boldsymbol{n}_{y}\left(k_{\mathbf{5}} \Delta l_{\mathbf{5}}+c_{\mathbf{5}} \Delta l_{\mathbf{5}}^{\prime}\right)-\boldsymbol{J}_{\omega \mathbf{4}}^{T} \boldsymbol{n}_{z}\left(k_{\mathbf{6}} \Delta l_{\mathbf{6}}+c_{\mathbf{6}} \Delta l_{\mathbf{6}}^{\prime}\right) \\
& \sum_{i=\mathbf{1}}^{\mathbf{4}}\left(m_{i 1} \boldsymbol{J}_{i \mathbf{1}}^{T} \boldsymbol{g}+m_{i 2} \boldsymbol{J}_{i 2}^{T} \boldsymbol{g}\right)+m_{P} \boldsymbol{J}_{P}^{T} \boldsymbol{g}+\boldsymbol{J}_{P}^{T} \boldsymbol{F}_{e}+\boldsymbol{J}_{\omega P}^{T} \boldsymbol{T}_{e}
\end{aligned}
$$

where $\Delta l_{4}=\Delta o_{x}, \Delta l_{5}=\Delta o_{y}, \Delta l_{6}=\Delta \psi . \Delta l_{4}^{\prime}=\Delta o_{x}^{\prime}, \Delta l_{5}^{\prime}=\Delta o_{y}^{\prime}$ and $\Delta l_{6}^{\prime}=\Delta \psi^{\prime}$. Substituting Eq. (82) and (67) into Kane's equation, the dynamics model can be re-expressed as

$$
\boldsymbol{J}_{k}\left[\begin{array}{c}
\Delta l_{1} \\
\Delta l_{2} \\
\Delta l_{3} \\
\Delta o_{x} \\
\Delta o_{y} \\
\Delta \psi
\end{array}\right]+\boldsymbol{J}_{c}\left[\begin{array}{c}
\Delta l_{1}^{\prime} \\
\Delta l_{2}^{\prime} \\
\Delta l_{3}^{\prime} \\
\Delta o_{x}^{\prime} \\
\Delta o_{y}^{\prime} \\
\Delta \psi^{\prime}
\end{array}\right]-\boldsymbol{G}\left(\boldsymbol{r}_{p}\right) \boldsymbol{g}-\boldsymbol{J}_{p}^{T} \boldsymbol{F}-\boldsymbol{J}_{\omega p}^{T} \boldsymbol{T}+\boldsymbol{M}\left(\boldsymbol{r}_{p}\right) \boldsymbol{r}_{p}^{\prime \prime}+\boldsymbol{M}^{\prime}\left(\boldsymbol{r}_{p}, \boldsymbol{r}_{p}^{\prime}\right) \boldsymbol{r}_{p}^{\prime}=\mathbf{0}
$$

where

$$
\begin{aligned}
& \boldsymbol{J}_{k}=\left[\begin{array}{llllllll}
k_{1} \boldsymbol{J}_{1}^{T} \boldsymbol{l}_{\mathbf{1}} & k_{2} \boldsymbol{J}_{2}^{T} \boldsymbol{l}_{\mathbf{2}} & k_{\mathbf{3}} \boldsymbol{J}_{\mathbf{3}}^{T} \boldsymbol{l}_{\mathbf{3}} \quad k_{\mathbf{4}} \boldsymbol{J}_{\mathbf{4}}^{T} \boldsymbol{n}_{x} \quad k_{\mathbf{5}} \boldsymbol{J}_{\mathbf{4}}^{T} \boldsymbol{n}_{y} \quad k_{\mathbf{6}} \boldsymbol{J}_{\omega \mathbf{4}}^{T} \boldsymbol{n}_{z}
\end{array}\right]^{T}, \\
& \boldsymbol{J}_{c}=\left[\begin{array}{lllllll}
c_{\mathbf{1}} \boldsymbol{J}_{\mathbf{1}}^{T} \boldsymbol{l}_{\mathbf{1}} & c_{\mathbf{2}} \boldsymbol{J}_{\mathbf{2}}^{T} \boldsymbol{l}_{\mathbf{2}} \quad c_{\mathbf{3}} \boldsymbol{J}_{\mathbf{3}}^{T} \boldsymbol{l}_{\mathbf{3}} \quad c_{\mathbf{4}} \boldsymbol{J}_{\mathbf{4}}^{T} \boldsymbol{n}_{x} \quad c_{\mathbf{5}} \boldsymbol{J}_{\mathbf{4}}^{T} \boldsymbol{n}_{y} \quad c_{\mathbf{6}} \boldsymbol{J}_{\omega \mathbf{4}}^{T} \boldsymbol{n}_{z}
\end{array}\right]^{T}, \\
& \boldsymbol{G}\left(\boldsymbol{r}_{p}\right)=\sum_{i=1}^{4}\left(m_{i 1} \boldsymbol{J}_{i \mathbf{1}}^{T}+m_{i 2} \boldsymbol{J}_{i 2}^{T}\right)+m_{P} \boldsymbol{J}_{P}^{T}, \\
& \boldsymbol{M}\left(\boldsymbol{r}_{p}\right)=\sum_{i=\mathbf{1}}^{4}\left[m_{i \mathbf{1}} \boldsymbol{J}_{i \mathbf{1}}^{T} \boldsymbol{J}_{i \mathbf{1}}+m_{i \mathbf{2}} \boldsymbol{J}_{i \mathbf{2}}^{T} \boldsymbol{J}_{i \mathbf{2}}+\boldsymbol{J}_{\omega i}^{T}\left(\boldsymbol{I}_{i \mathbf{1}}+\boldsymbol{I}_{i \mathbf{2}}\right) \boldsymbol{J}_{\omega i}\right]+m_{p} \boldsymbol{J}_{p}^{T} \boldsymbol{J}_{p}+\boldsymbol{J}_{\omega p}^{T} \boldsymbol{I}_{p} \boldsymbol{J}_{\omega p} \\
& \boldsymbol{M}^{\prime}\left(\boldsymbol{r}_{p}, \boldsymbol{r}_{p}^{\prime}\right)=\sum_{i=1}^{4}\left[m_{i \mathbf{1}} \boldsymbol{J}_{i \mathbf{1}}^{T} \boldsymbol{J}_{i \mathbf{1}}^{\prime}+m_{i \mathbf{2}} \boldsymbol{J}_{i \mathbf{2}}^{T} \boldsymbol{J}_{i \mathbf{2}}^{\prime}+\boldsymbol{J}_{\omega i}^{T}\left(\boldsymbol{I}_{i \mathbf{1}}+\boldsymbol{I}_{i \mathbf{2}}\right) \boldsymbol{J}_{\omega i}^{\prime}\right]+m_{p} \boldsymbol{J}_{p}^{T} \boldsymbol{J}_{p}^{\prime}+\boldsymbol{J}_{\omega p}^{T} \boldsymbol{I}_{p} \boldsymbol{J}_{\omega p}^{\prime} .
\end{aligned}
$$

Denoting the tiny deformation of the mobile platform by

$$
\Delta \boldsymbol{P}=[\Delta \alpha, \Delta \beta, \Delta \gamma, \Delta X, \Delta Y, \Delta Z]^{T}
$$

and substituting the Jacobian matrix in Eq. (50) into Eq. (83) yields

$$
\boldsymbol{K}\left(\boldsymbol{r}_{p}\right) \Delta \boldsymbol{P}+\boldsymbol{C}\left(\boldsymbol{r}_{p}\right) \Delta \boldsymbol{P}^{\prime}-\boldsymbol{G}\left(\boldsymbol{r}_{p}\right) \boldsymbol{g}-\boldsymbol{J}_{p}^{T} \boldsymbol{F}-\boldsymbol{J}_{\omega p}^{T} \boldsymbol{T}+\boldsymbol{M}\left(\boldsymbol{r}_{p}\right) \boldsymbol{r}_{p}^{\prime \prime}+\boldsymbol{M}^{\prime}\left(\boldsymbol{r}_{p}\right) \boldsymbol{r}_{p}^{\prime}=\mathbf{0},
$$


where $\boldsymbol{K}\left(\boldsymbol{r}_{p}\right)=\boldsymbol{J}_{k} \boldsymbol{J}$ and $\boldsymbol{C}\left(\boldsymbol{r}_{p}\right)=\boldsymbol{J}_{c} \boldsymbol{J}$. Since the displacement of the vibration is very small, we set $\Delta \boldsymbol{P}^{\prime}=\boldsymbol{r}_{p}^{\prime}$ and $\Delta \boldsymbol{P}^{\prime \prime}=\boldsymbol{r}_{p}^{\prime \prime}$ and Eq. (83) can be re-expressed as

$$
\boldsymbol{M}\left(\boldsymbol{r}_{p}\right) \Delta \boldsymbol{P}^{\prime \prime}+\left(\boldsymbol{C}\left(\boldsymbol{r}_{p}\right)+\boldsymbol{M}^{\prime}\left(\boldsymbol{r}_{p}, \boldsymbol{r}_{p}^{\prime}\right)\right) \Delta \boldsymbol{P}^{\prime}+\boldsymbol{K}\left(\boldsymbol{r}_{p}\right) \Delta \boldsymbol{P}-\boldsymbol{J}_{p}^{T} \boldsymbol{F}-\boldsymbol{J}_{\omega p}^{T} \boldsymbol{T}-\boldsymbol{G}\left(\boldsymbol{r}_{p}\right) \boldsymbol{g}=\mathbf{0} .
$$

Therefore, the vibration model of the 6-DOF vibration system is obtained.

\subsubsection{Considering the joint clearances}

Considering the linear relationships between the constraints and deformations of all branches and assuming that the passive branch is flexible, following the procedure of Eq. (86), the 6-DOF vibration model is

$$
\boldsymbol{M}\left(\boldsymbol{r}_{p}\right) \Delta \boldsymbol{P}^{\prime \prime}+\left(\boldsymbol{C}\left(\boldsymbol{r}_{p}\right)+\boldsymbol{M}^{\prime}\left(\boldsymbol{r}_{p}, \boldsymbol{r}_{p}^{\prime}\right)\right) \Delta \boldsymbol{P}^{\prime}+\boldsymbol{K}\left(\boldsymbol{r}_{p}\right) \Delta \boldsymbol{P}-\boldsymbol{J}_{p}^{T} \boldsymbol{F}-\boldsymbol{J}_{\omega p}^{T} \boldsymbol{T}-\boldsymbol{G}\left(\boldsymbol{r}_{p}\right) \boldsymbol{g}-\boldsymbol{J}_{k} \boldsymbol{d}=\mathbf{0},
$$

where $\boldsymbol{d}=\left[d_{1}, d_{2}, d_{3}, d_{4}, d_{5}, d_{6}\right]^{\mathrm{T}}$.

\subsection{Initial conditions}

In the study of vibration systems, the response of the mechanism under an actual external excitation is highly important as it directly affects the surface quality of the processed products; it is represented by the vibration amplitude. Since the vibration amplitude depends on the intensity of the initial disturbance, the initial conditions of vibration are important. For the drilling parallel robot, the initial conditions are as follows:

(1) The initial velocity is zero;

(2) The initial displacement is determined by the joint clearances.

Since the position and orientation attitude of the parallel robot are fixed during drilling, we assume that the initial velocity of the vibration system is zero. However, an assembly gap is unavoidable due to the mobility of the joints; hence, the mobile platform of the parallel robot has an initial displacement when vibrating. According to the principle of virtual work, the initial displacement can be calculated by solving

$$
\Delta \boldsymbol{P}_{\mathbf{0}}=\boldsymbol{J d},
$$

For the 3-DOF vibration system, $\boldsymbol{d}=\left[\begin{array}{lll}d_{1} & d_{2} & d_{3}\end{array}\right]^{T}$, which is specified in Section 3.1.2. For the 6-DOF vibration system, $\boldsymbol{d}=\left[\begin{array}{llllll}d_{1} & d_{2} & d_{3} & d_{4} & d_{5} & d_{6}\end{array}\right]^{T}$, which is also specified in Section 3.1.2.

\subsection{Mechanics of drilling}

As shown in Fig. 8, the mechanics of drilling mainly consists of two parts: the thrust that pushes the drill into the work material and the torque that causes the drill to rotate $[1,36]$. During the drilling process, the torsional force can be ignored because the torque is much smaller than the thrust. If we assume that the thrust is a cosine function with respect to time, the drilling force can be expressed as

Thrust $=F \cos \Omega t$.

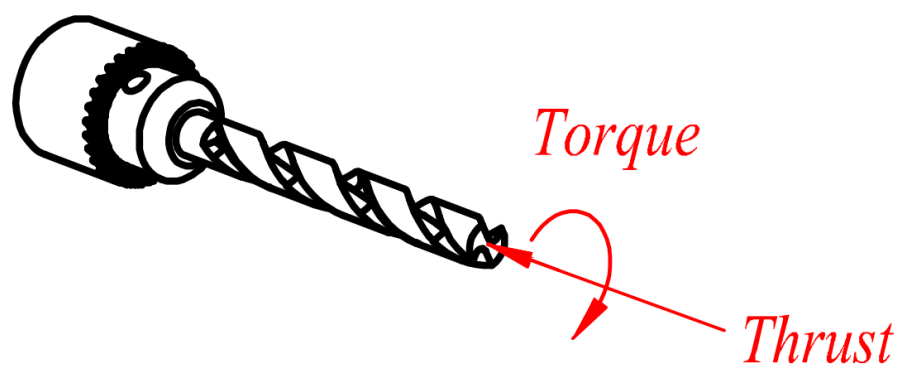

Fig. 8 Mechanics of drilling

Translating the drilling force into reference frame $O-X Y Z$ yields the following expression for the external load: 


$$
\boldsymbol{F}_{e}=\boldsymbol{R}\left[\begin{array}{c}
0 \\
0 \\
\text { Thrust }
\end{array}\right]=\left[\begin{array}{c}
F \sin \beta \cos \Omega t \\
-F \sin \alpha \cos \beta \cos \Omega t \\
F \cos \alpha \cos \beta \cos \Omega t
\end{array}\right]
$$

\section{Numerical examples, discussions and results}

\subsection{Parameter values}

For the following numerical examples, the mass value, geometric size and centroidal body inertia matrix of all components can be found in Ref. [35]. In addition, the joint clearances and the stiffnesses of all branches are listed in Tab. 1 and the damping of each branch is equal to 100 . The Coriolis matrix, namely, $\boldsymbol{M}^{\prime}\left(\boldsymbol{r}_{p}, \boldsymbol{r}_{p}^{\prime}\right)$, is neglected in the following vibration models because the amplitude of the vibration is very small and the Coriolis matrix is the quadratic of these magnitudes.

\begin{tabular}{cccc}
\multicolumn{4}{c}{ Tab. 1 Parameter values for the numerical examples } \\
\hline$d_{i}(i=1,2,3)(\mathrm{m})$ & $d_{4}(\mathrm{~m})$ & $d_{5}(\mathrm{~m})$ & $d_{6}(\mathrm{~m})$ \\
0.0003 & 0.0002 & 0.0002 & 0.0098 \\
$k_{i}(i=1,2,3)(\mathrm{N} / \mathrm{m})$ & $k_{4}(\mathrm{~N} / \mathrm{m})$ & $k_{5}(\mathrm{~N} / \mathrm{m})$ & $k_{6}(\mathrm{~N} / \mathrm{m})$ \\
$2.5000 \times 10^{8}$ & $1.4353 \times 10^{6}$ & $1.4353 \times 10^{6}$ & $2.6163 \times 10^{4}$ \\
\hline
\end{tabular}

\subsection{Natural frequencies}

As commonly used physical parameters, the natural frequencies reflect the inherent vibration characteristics of the system. To explore the natural frequencies of the system, we simplified the 3-DOF vibration system and the 6-DOF vibration system as follows:

$$
\boldsymbol{M}\left(\boldsymbol{r}_{p}\right) \Delta \boldsymbol{P}^{\prime \prime}+\boldsymbol{K}\left(\boldsymbol{r}_{p}\right) \Delta \boldsymbol{P}=\mathbf{0},
$$

Thus, the principle vibration mode can be calculated by solving

$$
\left(\boldsymbol{M}\left(\boldsymbol{r}_{p}\right)^{-1} \boldsymbol{K}\left(\boldsymbol{r}_{p}\right)-\omega^{2} \boldsymbol{E}_{3}\right) \boldsymbol{A}=\mathbf{0},
$$

where $\omega^{2}$ is the eigenvalue and $\omega$ is the natural frequency. Let $\boldsymbol{S}=\boldsymbol{M}\left(\boldsymbol{r}_{p}\right)^{-\mathbf{1}} \boldsymbol{K}\left(\boldsymbol{r}_{p}\right)$ and let $\boldsymbol{u}_{\mathrm{i}}$ be the corresponding eigenvector of $\omega^{2}$ of matrix $S$. The modal matrix can be determined via

$$
\Phi=\left[\mu_{1}, \mu_{2}, \mu_{3}\right]
$$

For the 3-DOF vibration system, three natural frequencies are obtained; they are plotted in Fig. 9. For the 6-DOF vibration system, six natural frequencies are obtained; they are plotted in Fig. 10. For convenience of description, the first three frequencies of the 6-DOF vibration system are called the parasitic natural frequencies $(P N F s)$ and the three vibration frequencies of the 3-DOF vibration system $\left(\omega_{1}, \omega_{2}\right.$ and $\left.\omega_{3}\right)$ and the last three vibration frequencies of the 6-DOF vibration system $\left(\omega_{4}, \omega_{5}\right.$ and $\left.\omega_{6}\right)$ are called the ideal natural frequencies (INFs). The considered workspace is described by the location of the end of the twist drill and the coordinate of the Z-axis is $1.08 \mathrm{~m}$. Due to the higher driving stiffness value, the natural frequency value obtained in this numerical example is higher. When the adopted driving stiffness value is $2.5 \times 106 \mathrm{~N} / \mathrm{m}$, The three natural frequencies of the 3-DOF system and the last three natural frequencies of the 6-DOF system are given separately in Fig. 11(a) and Fig. 11(b).

\subsection{Forced vibrations}

The amplitude can directly reflect the vibration characteristics of the parallel robots in various directions; hence, the forced vibration of the system under the initial conditions is studied in this section. Ignoring the Coriolis matrix, the forced vibration models of the two systems can be simplified as

$$
\boldsymbol{M}\left(\boldsymbol{r}_{p}\right) \Delta \boldsymbol{P}^{\prime \prime}+\boldsymbol{C}\left(\boldsymbol{r}_{p}\right) \Delta \boldsymbol{P}^{\prime}+\boldsymbol{K}\left(\boldsymbol{r}_{p}\right) \Delta \boldsymbol{P}-\boldsymbol{G}\left(\boldsymbol{r}_{p}\right) \boldsymbol{g}-\boldsymbol{J}_{p}^{T} \boldsymbol{F}_{e}-\boldsymbol{J}_{\omega p}^{T} \boldsymbol{T}_{e}-\boldsymbol{J}_{k} \boldsymbol{d}=\mathbf{0}
$$

Multiplying by $\boldsymbol{\Phi}^{T}$ on both sides of Eq. (94) yields 


$$
\boldsymbol{\Phi}^{T} \boldsymbol{M}\left(\boldsymbol{r}_{p}\right) \Delta \boldsymbol{P}^{\prime \prime}+\boldsymbol{\Phi}^{T} \boldsymbol{C}\left(\boldsymbol{r}_{p}\right) \Delta \boldsymbol{P}^{\prime}+\boldsymbol{\Phi}^{T} \boldsymbol{K}\left(\boldsymbol{r}_{p}\right) \Delta \boldsymbol{P}-\boldsymbol{\Phi}^{T} \boldsymbol{G}\left(\boldsymbol{r}_{p}\right) \boldsymbol{g}-\boldsymbol{\Phi}^{T} \boldsymbol{J}_{p}^{T} \boldsymbol{F}_{e}-\boldsymbol{\Phi}^{T} \boldsymbol{J}_{\omega p}^{T} \boldsymbol{T}_{e}-\boldsymbol{\Phi}^{T} \boldsymbol{J}_{k} \boldsymbol{d}=\mathbf{0}
$$

Let $\Delta \boldsymbol{P}=\boldsymbol{\Phi} \boldsymbol{u}, \Delta \boldsymbol{P}^{\prime}=\boldsymbol{\Phi} \boldsymbol{u}^{\prime}$, and $\Delta \boldsymbol{P}^{\prime \prime}=\boldsymbol{\Phi} \boldsymbol{u}^{\prime}$. The previous equation can be re-expressed as

$$
\boldsymbol{\Phi}^{T} \boldsymbol{M}\left(\boldsymbol{r}_{p}\right) \boldsymbol{\Phi} \boldsymbol{u}^{\prime \prime}+\boldsymbol{\Phi}^{T} \boldsymbol{C}\left(\boldsymbol{r}_{p}\right) \boldsymbol{\Phi} \boldsymbol{u}^{\prime}+\boldsymbol{\Phi}^{T} \boldsymbol{K}\left(\boldsymbol{r}_{p}\right) \boldsymbol{\Phi} \boldsymbol{u}-\boldsymbol{\Phi}^{T} \boldsymbol{G}\left(\boldsymbol{r}_{p}\right) \boldsymbol{g}-\boldsymbol{\Phi}^{T} \boldsymbol{J}_{p}^{T} \boldsymbol{F}_{e}-\boldsymbol{\Phi}^{T} \boldsymbol{J}_{\omega P}^{T} \boldsymbol{T}_{e}-\boldsymbol{\Phi}^{T} \boldsymbol{J}_{k} \boldsymbol{d}=\mathbf{0}
$$

Matrices $\boldsymbol{\Phi}^{T} \boldsymbol{M}\left(\boldsymbol{r}_{p}\right) \boldsymbol{\Phi}, \boldsymbol{\Phi}^{T} \boldsymbol{K}\left(\boldsymbol{r}_{p}\right) \boldsymbol{\Phi}$ and $\boldsymbol{\Phi}^{T} \boldsymbol{C}\left(\boldsymbol{r}_{p}\right) \boldsymbol{\Phi}$ are diagonal matrices and the previous equation can be simplified to a standing vibration equation as

$$
\boldsymbol{M} \boldsymbol{u}^{\prime \prime}+\boldsymbol{C} \boldsymbol{u}^{\prime}+\boldsymbol{K u}=\boldsymbol{G}+\boldsymbol{D}+\boldsymbol{F}+\boldsymbol{T}
$$

where $\boldsymbol{M}=\boldsymbol{\Phi}^{T} \boldsymbol{M}\left(\boldsymbol{r}_{p}\right) \boldsymbol{\Phi}, \boldsymbol{K}=\boldsymbol{\Phi}^{T} \boldsymbol{K}\left(\boldsymbol{r}_{p}\right) \boldsymbol{\Phi}, \boldsymbol{C}=\boldsymbol{\Phi}^{T} \boldsymbol{C}\left(\boldsymbol{r}_{p}\right) \boldsymbol{\Phi}, \boldsymbol{G}=\boldsymbol{\Phi}^{T} \boldsymbol{G}\left(\boldsymbol{r}_{p}\right) \boldsymbol{g}, \boldsymbol{F}=\boldsymbol{\Phi}^{T} \boldsymbol{J}_{p}^{T} \boldsymbol{F}_{e}, \boldsymbol{T}=\boldsymbol{\Phi}^{T} \boldsymbol{J}_{\omega p}^{T} \boldsymbol{T}_{e}$ and $\boldsymbol{D}=\boldsymbol{\Phi}^{T} \boldsymbol{J}_{k} \boldsymbol{d}$. Substituting the initial conditions into Eq. (57), the solutions of this standing vibration equation are

$$
u_{i}=h_{i 1} e^{s_{1} t}+h_{i 2} e^{s_{2} t}+h_{i 3} \sin (\Omega t)+h_{i 4} \cos (\Omega t)+\frac{G_{i 1}+D_{i 1}}{K_{i i}},
$$

where

$$
\begin{aligned}
& s_{i 1, i 2}=\frac{-C_{i i} \pm \sqrt{C_{i i}^{2}-\mathbf{4} M_{i i} K_{i i}}}{2 M_{i i}}, h_{i 3}=\frac{F_{i 1} C_{i 1} \Omega}{\left(K_{i i}-M_{i i} \Omega^{2}\right)^{2}+C_{i i}^{2} \Omega^{2}}, h_{i 4}=\frac{F_{i 1}\left(K_{i i}-M_{i i} \Omega^{2}\right)}{\left(K_{i i}-M_{i i} \Omega^{2}\right)^{2}+C_{i i}^{2} \Omega^{2}}, \\
& h_{i 1}=\frac{-s_{i 2} u_{0 i}+s_{i 2} h_{i 4}+s_{i 2} \frac{G_{i 1}+D_{i 1}}{K_{i i}}-h_{i 3} \Omega}{s_{i 1}-s_{i 2}}, h_{i 2}=\frac{s_{i 1} u_{0 i}-s_{i 1} h_{i 4}-s_{i 1} \frac{G_{i 1}+D_{i 1}}{K_{i i}}+h_{i 3} \Omega}{s_{i 1}-s_{i 2}}, \boldsymbol{u}_{0}=\boldsymbol{\Phi}^{-1} \Delta \boldsymbol{P}_{0},
\end{aligned}
$$

(a)

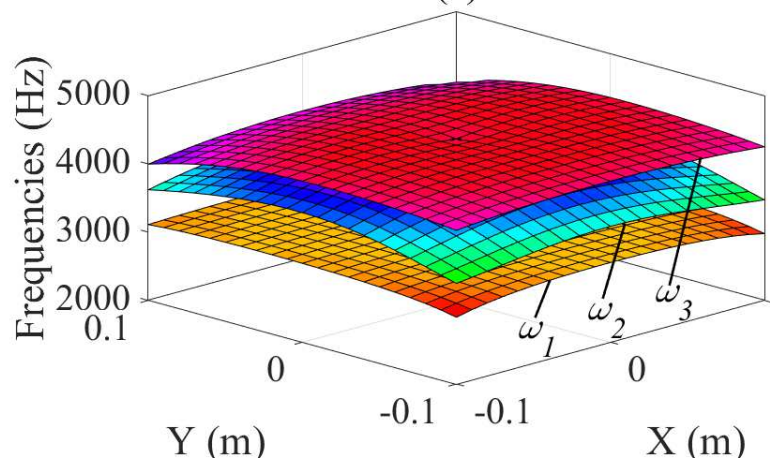

(c)

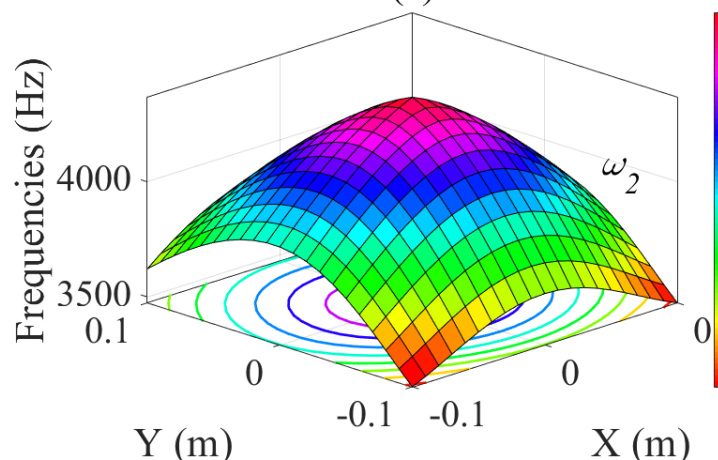

Fig. 9 Natural frequencies of the 3-DOF vibration system: (b)

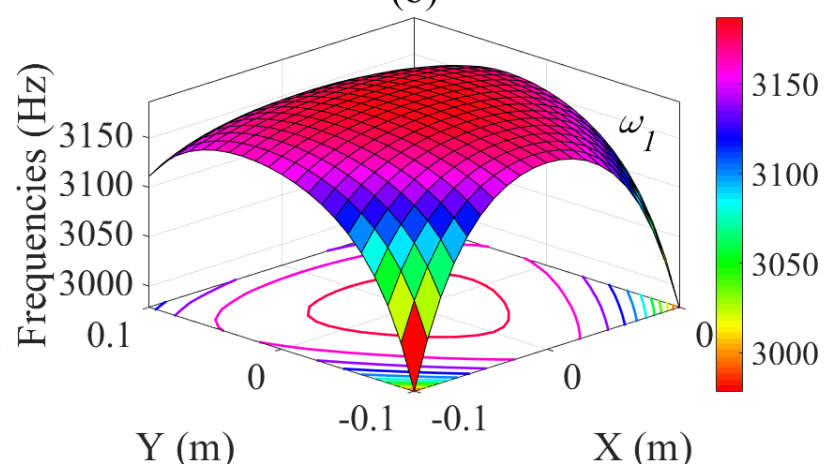

(d)

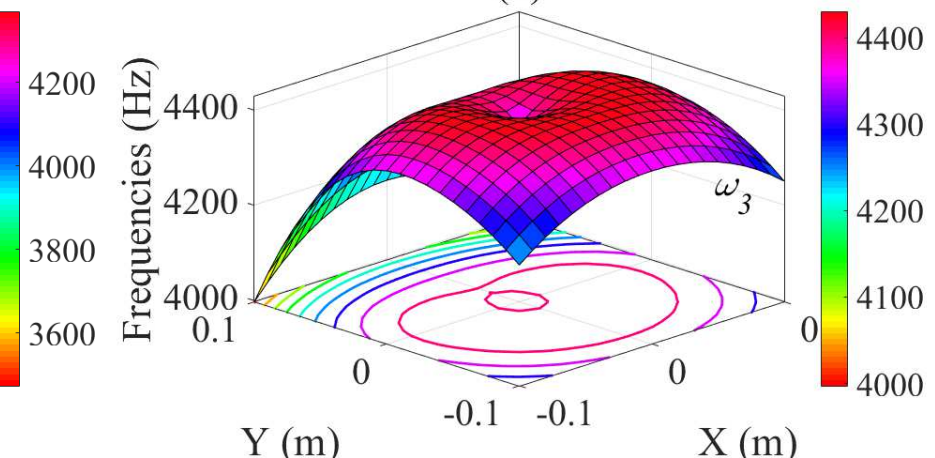

$\mathrm{Y}(\mathrm{m})$

(a) The three ideal natural frequencies; (b) (d) the first through third natural frequencies. 
(a)

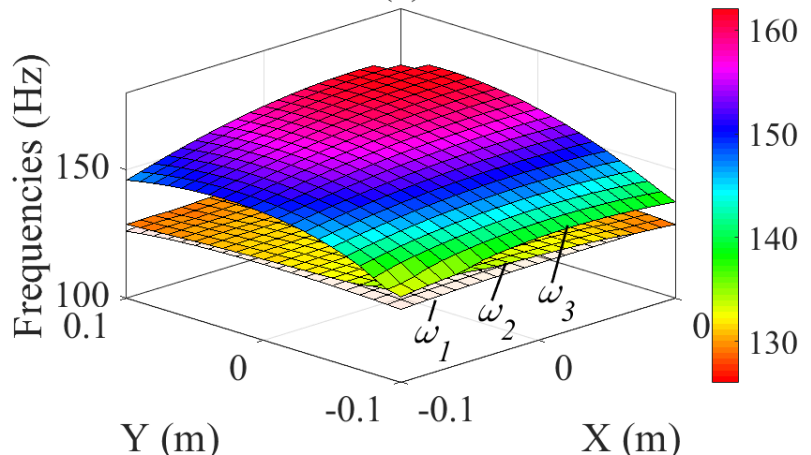

(c)

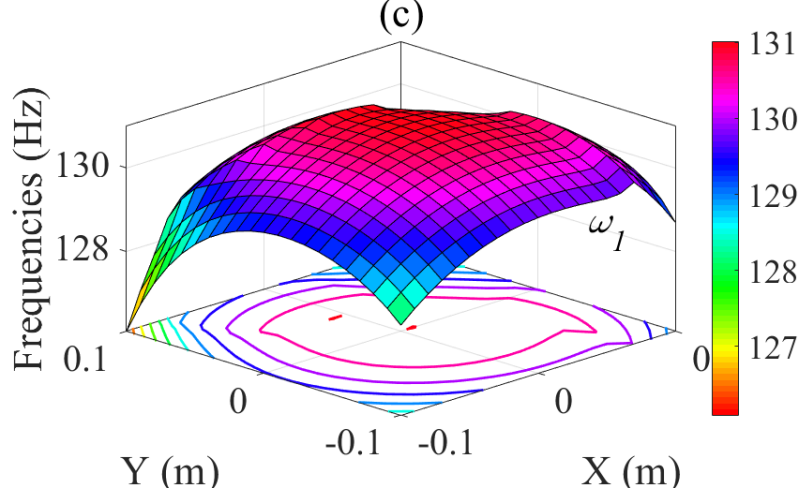

(e)

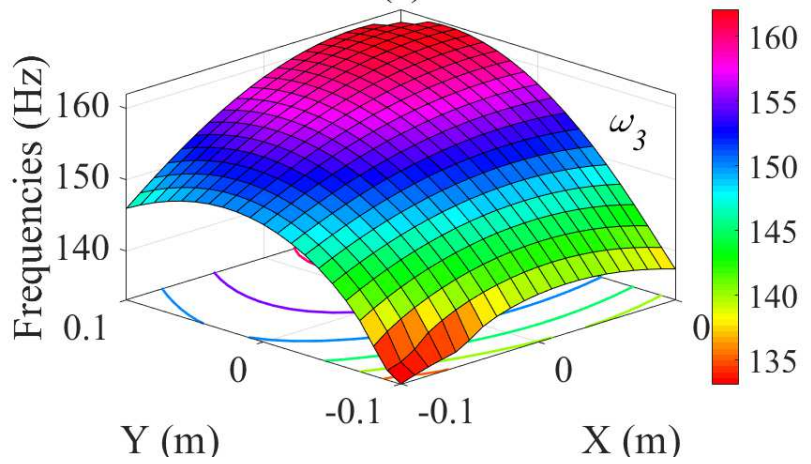

$(\mathrm{g})$

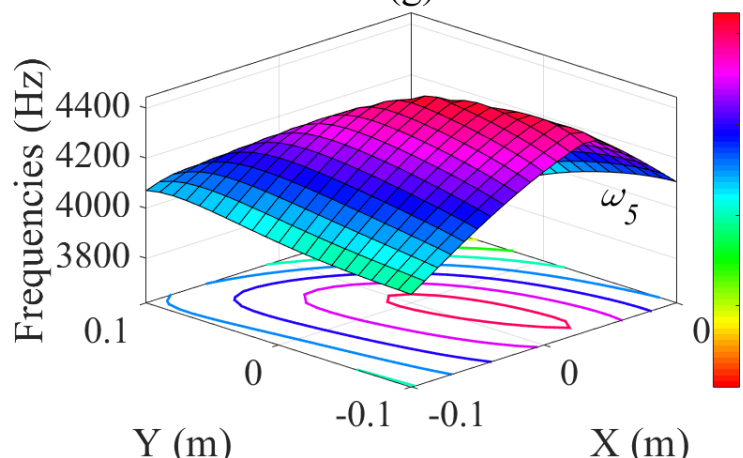

(b)

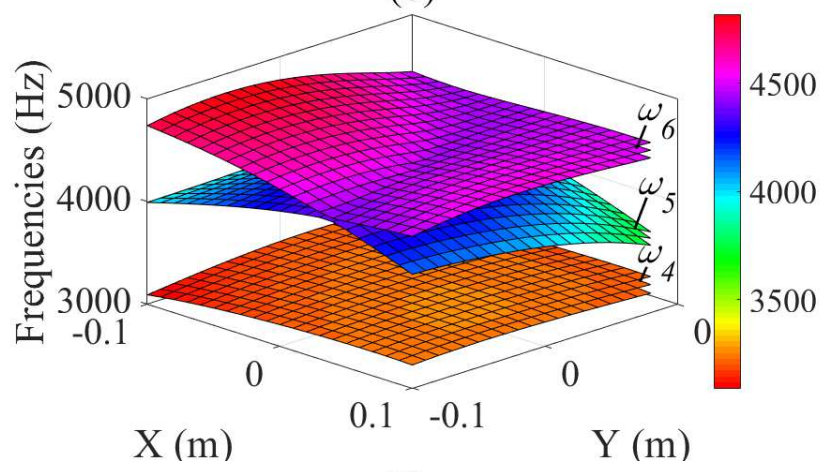

(d)

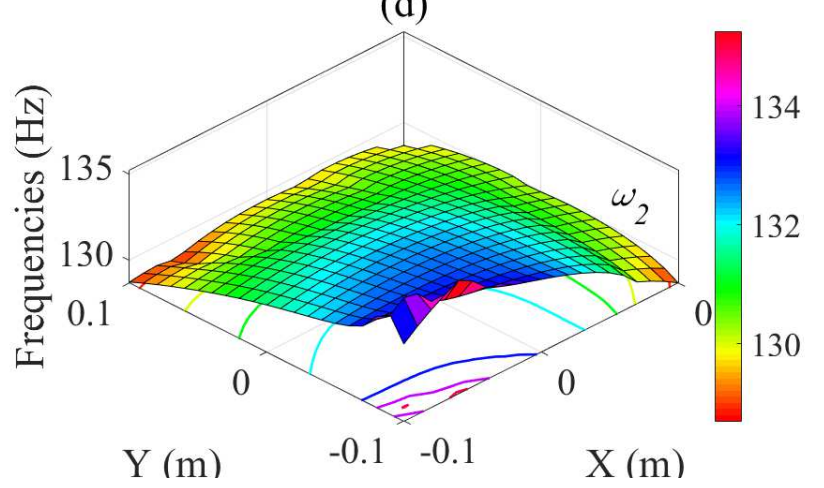

(f)

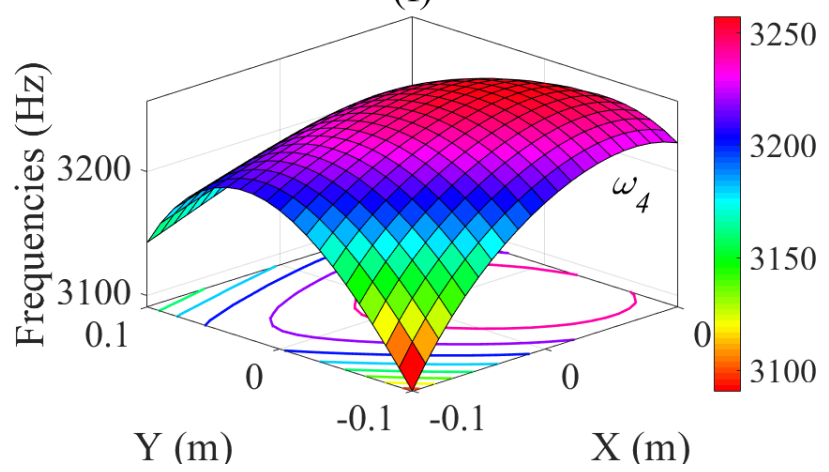

(h)

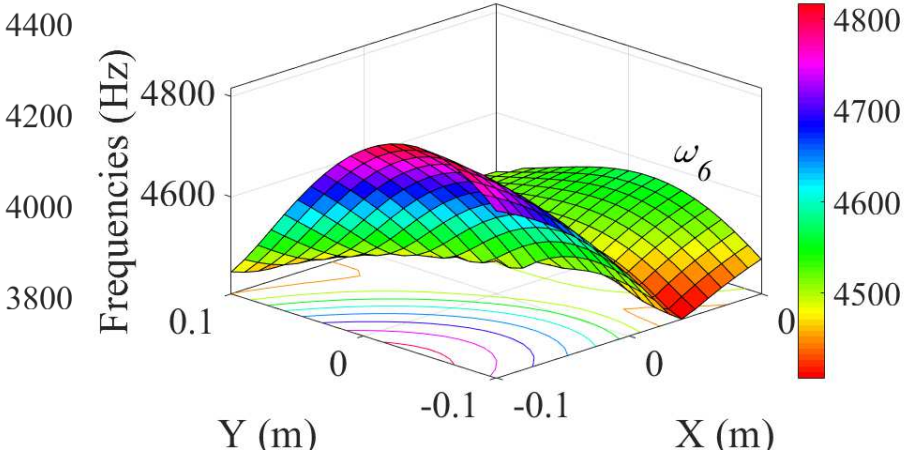

Fig. 10 Natural frequencies of the 6-DOF vibration system: (a) The three ideal natural frequencies; (b) the three parasitic natural frequencies; and (c) (h) the first through sixth natural frequencies.

in which the subscripts of the uppercase letters represent the elements in their corresponding matrices. Finally, substituting the parameter values into the vibration equation, the vibration amplitudes of the 3-DOF and 6-DOF 
(a)

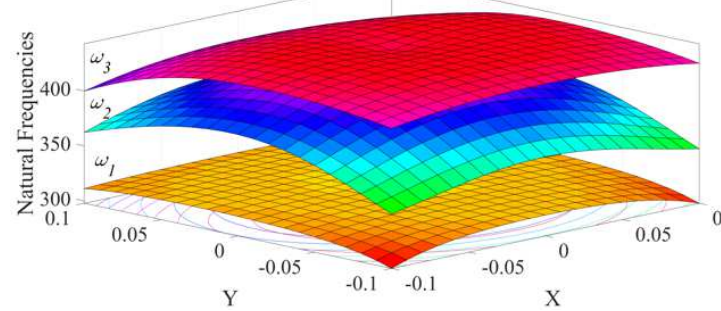

(b)

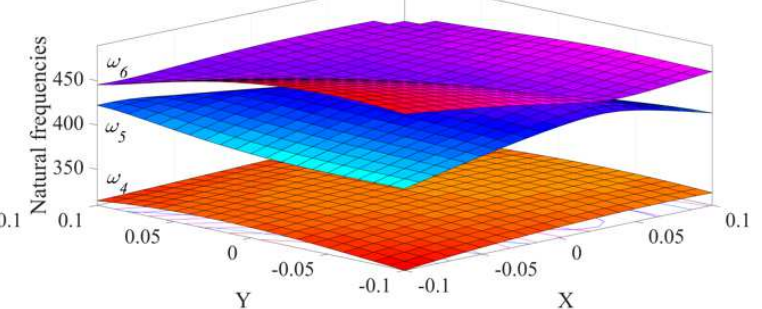

Fig. 11 The natural frequency of the system that changes the value of the driving stiffness: (a) 3-DOF system; (b) 6-DOF system vibration systems can be solved via $\Delta \boldsymbol{P}=\boldsymbol{\Phi} \boldsymbol{u}$ and are plotted in Fig. 12 and Fig. 13, respectively. For the 3-DOF vibration system, the vibrations of $\Delta \alpha, \Delta \beta$, and $\Delta l_{4}$ are called the three original vibrations. For the 6-DOF vibration system, the vibrations of $\Delta \alpha, \Delta \beta$, and $\Delta Z$ are called the three original vibrations and those of $\Delta X, \Delta Y$ and $\Delta \gamma$ are called the three parasitic vibrations.
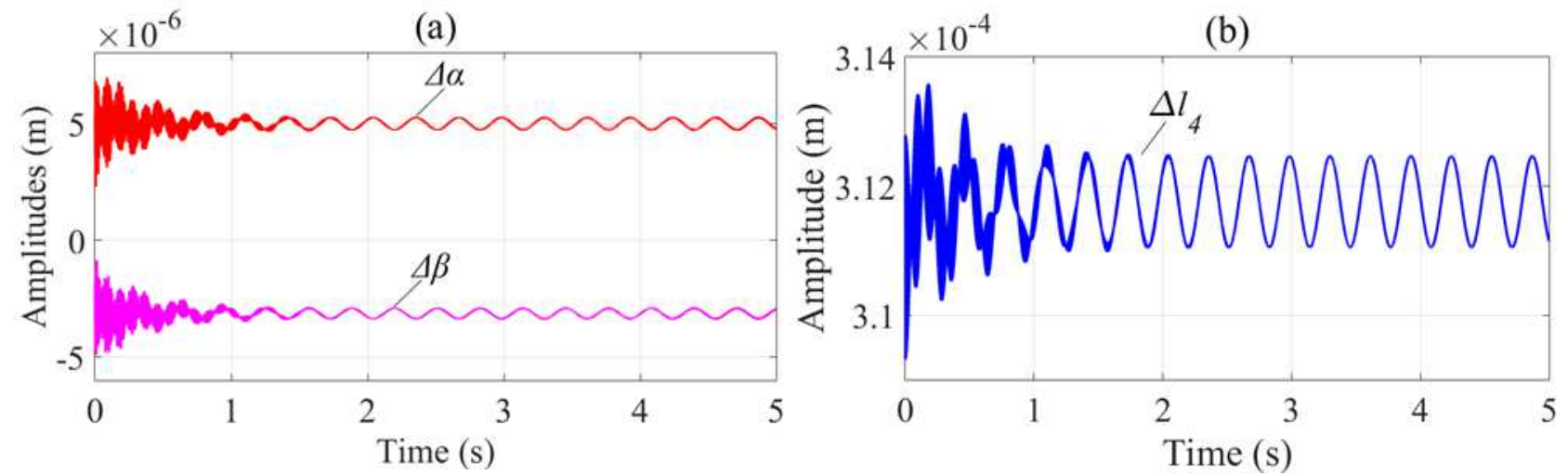

Fig. 12 Forced vibration amplitudes of the 3-DOF vibration system: (a) two rotational vibrations; (b) one translational vibration.
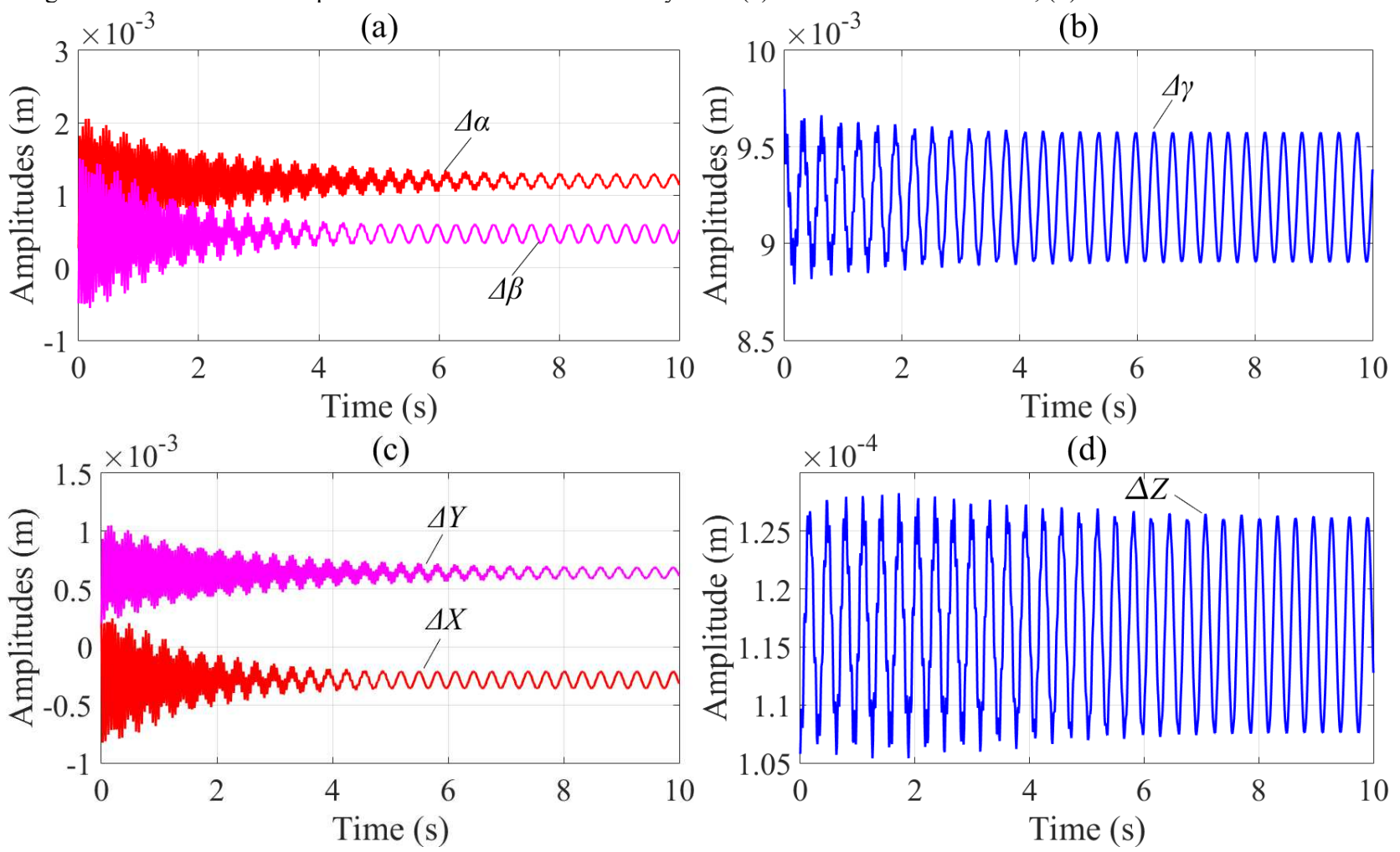

Fig. 13 Forced vibration amplitudes of the 6-DOF vibration system: (a) rotational vibrations around the $X$-axis and the $Y$-axis; (b) rotational vibration around the $Z$-axis; (c) translational vibrations along the $X$-axis and the $Y$-axis; (d) translational vibration along the $Z$-axis. 


\subsection{Comparisons of the two vibration systems}

The natural frequencies of the two vibration systems are compared. According the numerical results of Section 4.2, the 3-DOF vibration system has three natural frequencies, while the 6-DOF vibration system has six natural frequencies. The three $I N F \mathrm{~s}$ of the 3-DOF vibration system are similar to the three $I N F \mathrm{~s}$ of the 6-DOF vibration system, whereas the three $P N F$ s of the 6-DOF vibration system are much smaller. In traditional studies, the actual 6-DOF vibration system is considered an ideal 3-DOF vibration system; hence, the smaller three $P N F \mathrm{~s}$ of the 6DOF vibration system are ignored. For a clearer comparison of this idealization process, the natural frequencies of the point $(0.1,0.1,1.08)$ are listed in Tab. 2 and the minimum natural frequencies of the three $I N F \mathrm{~s}$ of the two systems are compared in Fig. 14, where the minimum natural frequency of the 6-DOF vibration system is represented by $\omega_{4}$. The distribution of the minimum natural frequency of the 3-DOF vibration system is symmetric about the Y-axis, whereas this distribution is not applicable for the 6-DOF vibration system and the value difference varies from 10 to 100 among locations. The effects of the three parasitic motions are important factors for this phenomenon. In some scenarios, this idealization process is applicable since the difference is small. However, if the vibration frequency of the external excitation is close to the three $P N F \mathrm{~s}$, the vibration defects that are caused by the ideal 3-DOF vibration system will be observed.

Tab. 2 Natural frequencies of two systems

$(\mathrm{Hz})$

\begin{tabular}{lllllll}
\hline & $\omega_{1}$ & $\omega_{2}$ & $\omega_{3}$ & $\omega_{4}$ & $\omega_{5}$ & $\omega_{6}$ \\
\hline 3-DOFs & 3111.7 & 3.6220 & 3.9973 & & & \\
6-DOFs & 126.4 & 129.0 & 160.6 & 3174.0 & 3527.8 & 4490.7 \\
\hline
\end{tabular}

(a)

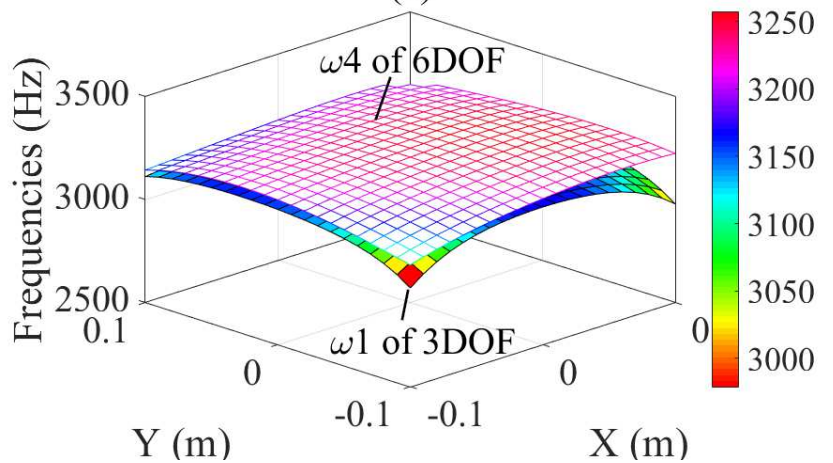

Fig. 14 Comparisons of natural frequencies: (a) graphic model; (b) projection drawing. (b)

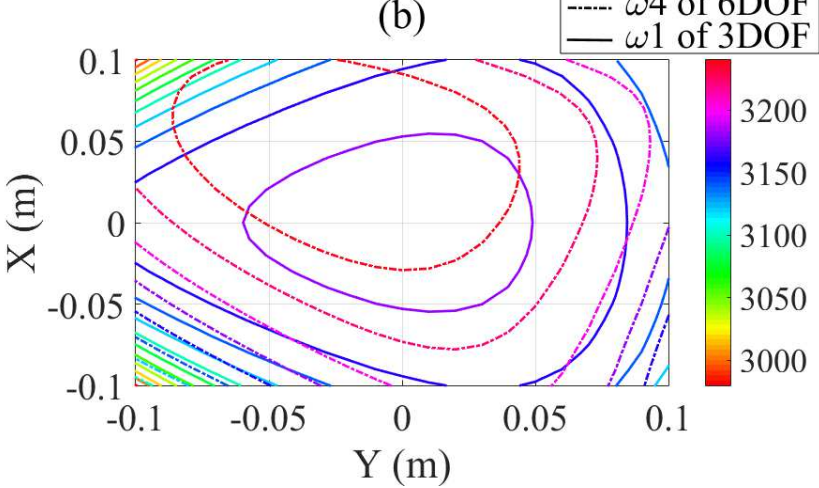

Then, the vibration behaviours of the two vibration systems are compared. According to the numerical results of Section 4.2, in the directions of the three parasitic motions, the amplitudes of the 3-DOF vibration system are null, whereas the amplitudes of the 6-DOF vibration system cannot be ignored. As shown in Fig. 15, in the directions of the three original motions, the amplitudes of the two vibration systems differ, and the rotation motion around the $X$-axis is taken as an instant to reveal the difference, as presented in Tab. 3. For the 3-DOF vibration system, the initial amplitude of the forced vibration that is caused by the initial conditions is approximately $6 \times 10^{-6} \mathrm{~m}$, the steadystate amplitude is approximately $1 \times 10^{-6} \mathrm{~m}$, and the transition time (from the initial vibration to the steady-state vibration) is approximately $1.5 \mathrm{~s}$. For the 6-DOF vibration system, the initial amplitude is approximately $1.5 \times 10^{-3}$ $\mathrm{m}$, the steady-state amplitude is approximately $3 \times 10^{-4}$, and the transition time is approximately $7 \mathrm{~s}$. Therefore, the vibration behaviours of the two vibration systems differ substantially.

Tab. 3 Amplitudes of two systems around the X-axis

\begin{tabular}{cccc}
\hline & Initial amplitude $(\mathrm{m})$ & Steady-state amplitude $(\mathrm{m})$ & Transition time $(\mathrm{s})$ \\
\hline 3-DOFs & $3 \times 10^{-6}$ & $1 \times 10^{-6}$ & 1.5 \\
6-DOFs & $7.5 \times 10^{-4}$ & $1.5 \times 10^{-4}$ & 7 \\
\hline
\end{tabular}



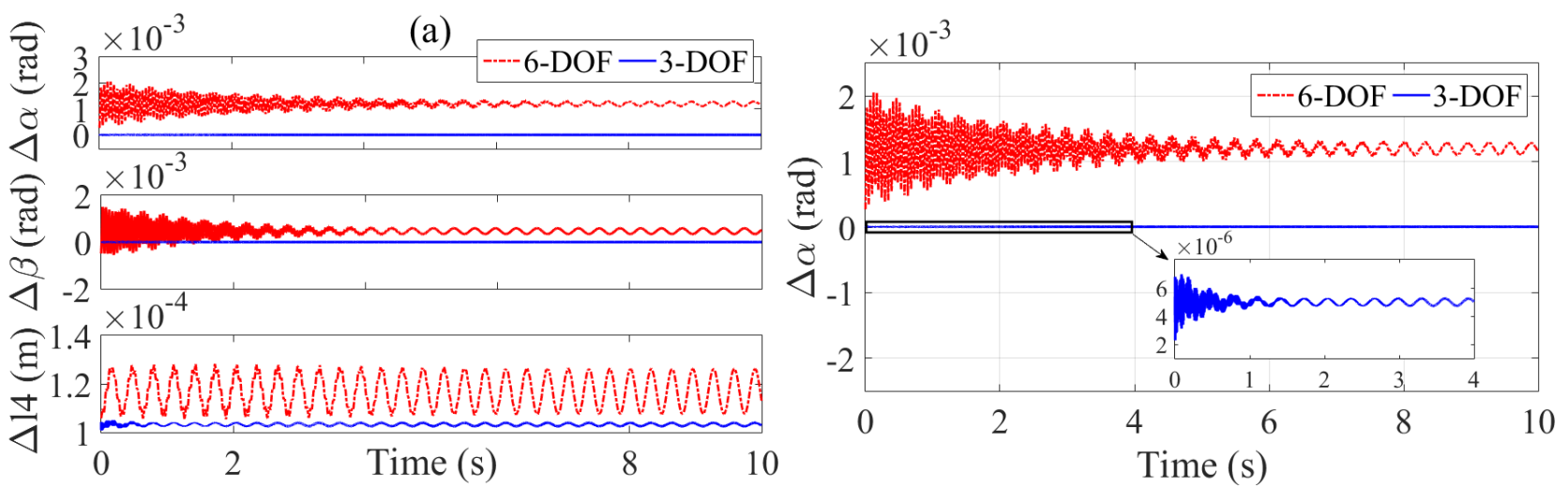

Fig. 15 Vibration behaviour comparisons between two vibration systems: (a) three original motions; (b) around the $X$-axis.

\subsection{Effects of the joint clearances}

To explore the effects of the joint clearances, the 6-DOF vibration system, which is more consistent with the actual system, is used as an example. The numerical results for the scenarios in which joint clearances are considered and joint clearances are ignored are shown in Fig. 16 and the rotation motion around the $X$-axis is taken as an instant to reveal the difference; the results are presented in Fig. 17 and Tab. 4. If the joint clearances are considered, the initial amplitude is approximately $7.5 \times 10^{-4} \mathrm{~m}$, the steady-state amplitude is approximately $1.5 \times 10^{-4}$, the vibration balanced location is $1.2 \times 10^{-3} \mathrm{~m}$, and the transition time is approximately $7 \mathrm{~s}$. If the joint clearances are ignored, the initial amplitude is approximately $2.8 \times 10^{-3} \mathrm{~m}$, the steady-state amplitude is approximately $1.5 \times 10^{-4}$, the vibration balanced location is $0 \mathrm{~m}$, and the transition time is also approximately $7 \mathrm{~s}$. In other directions, although the values may vary, the differences in the vibration law are approximately same. Therefore, the following results are obtained from the joint clearance comparison: the amplitude difference in the initial vibration phase is huge, the steady-state vibration law remains approximately the same, the transition time remains approximately the same, and the balanced location is translated.
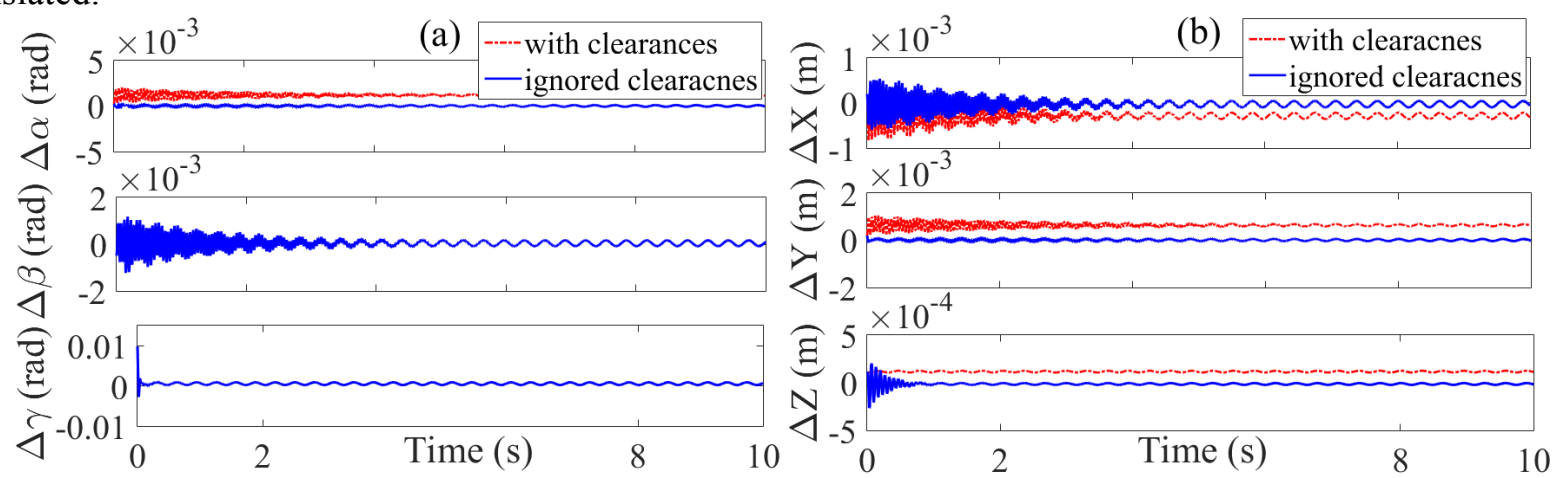

Fig. 16 Effects of the joint clearances on the 6-DOF vibration system: (a) rotational vibrations; (b) translational vibrations.

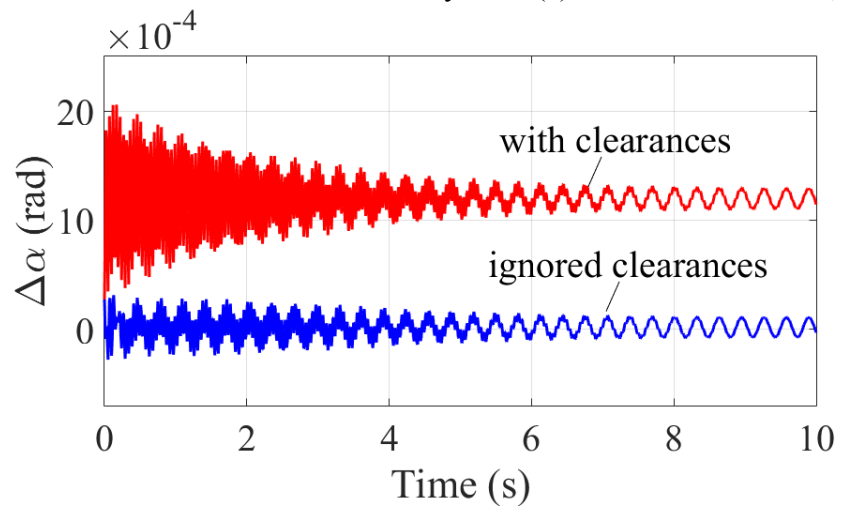

Fig. 17 Effects of the joint clearances around the $\mathrm{X}$-axis direction 
Tab. 4 Amplitudes of two systems around the X-axis

\begin{tabular}{|c|c|c|c|c|}
\hline & Initial amplitude (m) & Steady-state amplitude (m) & Balanced location (m) & Transition time $(\mathrm{s})$ \\
\hline With joint clearances & $7.5 \times 10^{-4}$ & $1.5 \times 10^{-4}$ & $1.2 \times 10^{-3}$ & 7 \\
\hline Ignoring joint clearances & $2.8 \times 10^{-4}$ & $1.5 \times 10^{-4}$ & 0 & 7 \\
\hline
\end{tabular}

\subsection{Effects of damping and gravity}

As shown in Fig. 18, the rotation motion around the $X$-axis is taken as an instant to evaluate the effects of the damping and gravity. As the damping value is changed, the initial amplitude and the steady-state amplitude remain constant, whereas the transition time changes: if the damping is larger, the transition time is shorter. If the damping is ignored, the amplitude of the vibration will not change from the initial amplitude, which does not match the actual scenario. Moreover, comparing with the vibration system in which gravity is ignored, the initial amplitude, the steady-state amplitude and the transition time of the vibration system in which gravity is considered are same, whereas the vibration balanced location changes. If the magnitude of the vibration amplitude is smaller, the effect of gravity on the vibration balanced location is stronger.
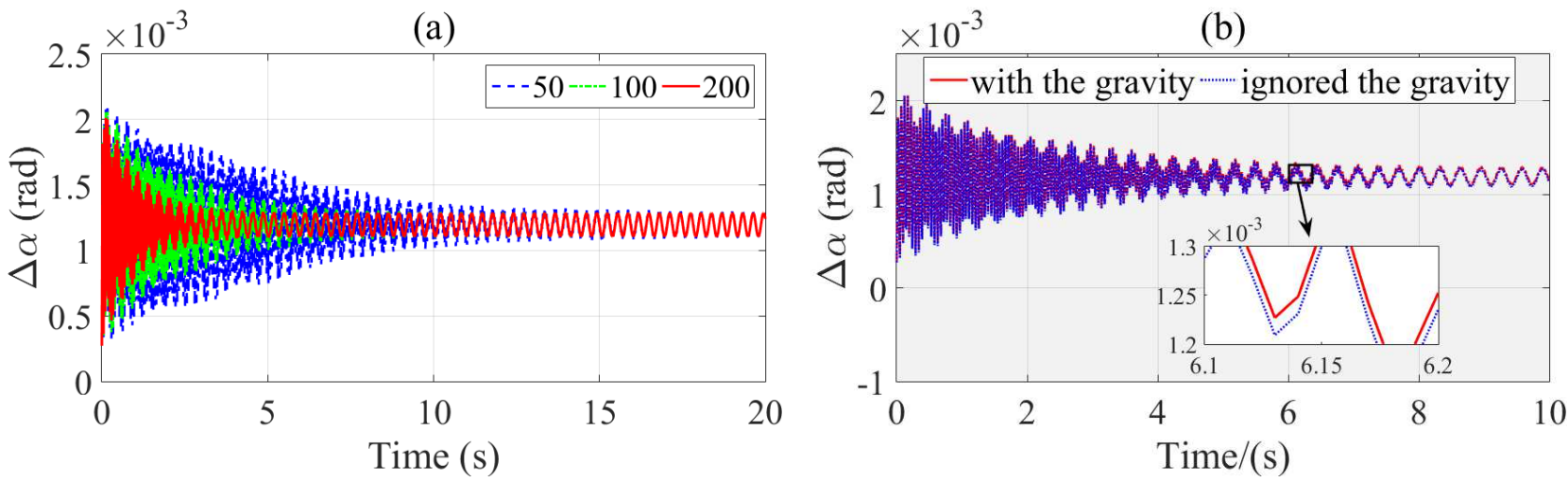

Fig. 18 Effects of damping and gravity: (a) damping; (b) gravity.

\subsection{Results}

For the drilling parallel robot, we consider the 3-DOF vibration system in which the effects of the passive branch, gravity and the joint clearances are ignored as the ideal model and consider the 6-DOF vibration system in which all effects are considered as the actual vibration model. The comparison results of vibration motion around the $X$ axis by the software of MATLAB are shown in Fig. 19. The minimum natural frequency of the ideal vibration model is approximately 30 times the minimum natural frequency of the actual vibration model, the initial and steady-state amplitudes of the ideal vibration model are less than $1 \%$ of the initial and steady-state amplitudes of the actual vibration model, the transition time of the ideal vibration model is $78 \%$ shorter than the transition time of the actual vibration model, and the balanced location of the vibration is translated by $1.2 \mathrm{~mm}$. Moreover, in order to verify the correctness of the vibration models, the finite element analysis software of ABAQUS is used to simulate the drilling parallel robot. The simulation results of the first natural frequencies are shown in Fig. 20, where the passive branch is rigid for the 3-DOF vibration system and is flexible for the 6-DOF vibration system. Although the simulation results of ABAQUS differ from the theoretical values solved by MATLAB, which may be caused by the differences of joint clearances and geometry, their laws of change are the same, and it prove that our vibration analysis is correct in some extent. As a result, five clear results are summarized as follows:

a) The natural frequency is affected by the passive branch. If the passive branch is considered rigid, the three $P N F \mathrm{~s}$, which are much smaller than the three $I N F \mathrm{~s}$, will be neglected and the distribution and values of the three $I N F \mathrm{~s}$ will differ from the actual distribution and values;

b) The natural frequency is independent of the joint clearances;

c) The vibration behaviour is affected by the passive branch. If the passive branch is considered rigid, the ignored three parasitic vibrations are important along with the three original vibrations and the initial amplitudes, steadystate amplitudes and transition times of the three original vibrations differ from the actual values; 
d) The vibration behaviour is affected by the joint clearances. The steady-state amplitude and the transition time are independent of the joint clearances. However, the initial amplitude and the balanced location differ from the actual values;

e) The balanced location of vibration is affected by gravity.

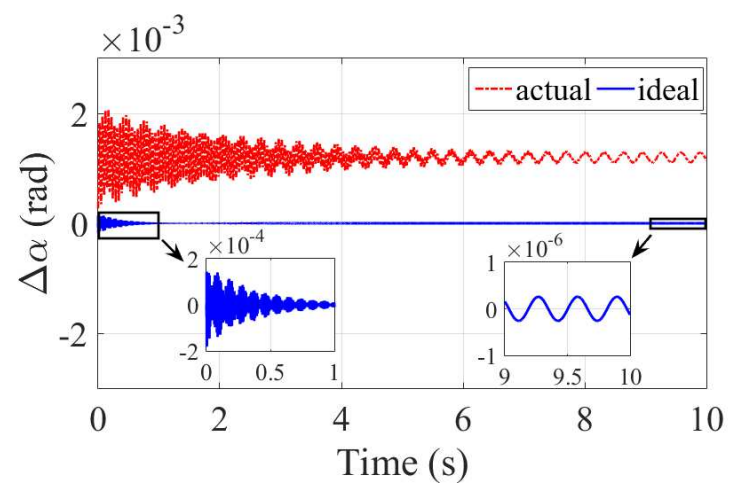

Fig. 19 Comparison between the actual vibration model and the ideal vibration model

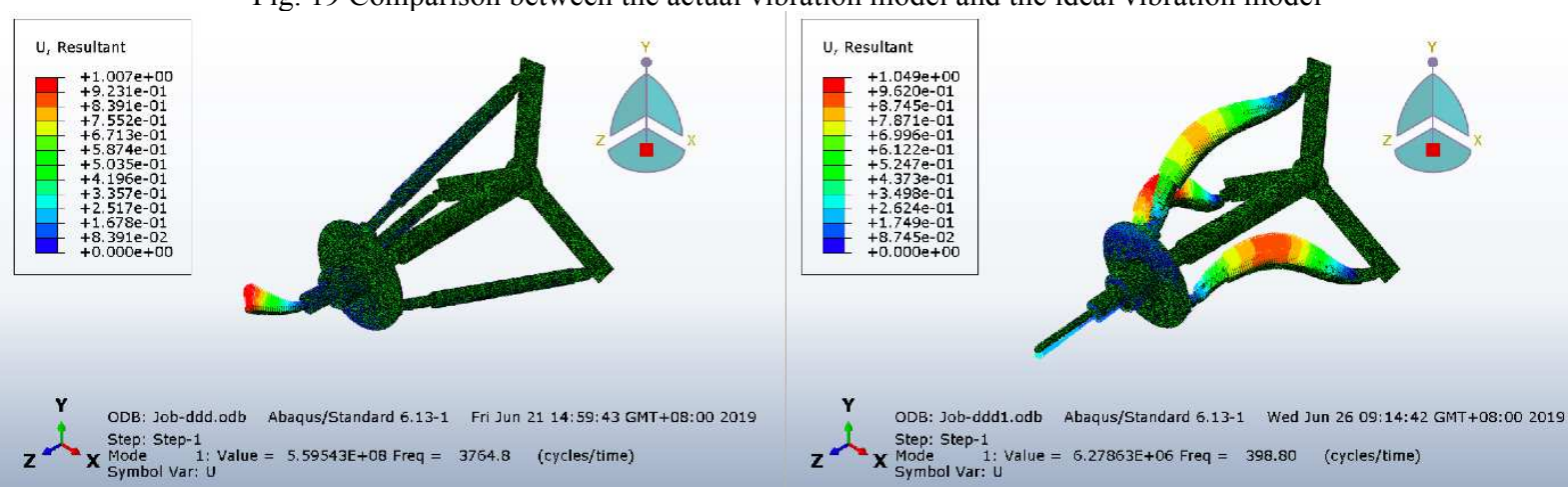

Fig. 20 The first natural frequencies solved by ABAQUS: (a) the 3-DOF vibration system; (b) the 6-DOF vibration system.

\section{Conclusions}

In this paper, vibration modelling with $3-\mathrm{DOF}$ and $6-\mathrm{DOF}$ models for a $1 \mathrm{PU}+3 \mathrm{UPS}$ parallel robot is carried out, and the accuracy of the traditional simplified vibration model is discussed. In the proposed vibration modelling method, if the velocities and accelerations of all components are expressed in a fixed form, the vibration model of parallel robots can be easily deduced. Based on the results and discussions, the main reason the designed prototype cannot satisfy the design requirements is that the influences of the passive branches and joint clearances were neglected in the previous vibration optimization design process. The passive branch will affect the number of the natural frequency; the magnitude and distribution of the natural frequency; the initial and steady-state amplitudes of vibration; and the transition time of vibration. The joint clearances will affect the initial amplitude and balanced location of the vibration. Gravity will affect the vibration-balanced location, and the damping will affect the transition time of vibration. In the design process of high-precision parallel robots, the proposed vibration model should be fully considered, including the effects of the passive branch, joint clearances, gravity, and damping, and the vibration performance cannot be judged solely according to the natural frequency. Furthermore, researchers can also judge whether these factors can be ignored based on these effects.

\section{Competing interests}

The authors declare no competing financial interests. 


\section{Funding}

This work was supported by the National Natural Science Foundation of China [grant numbers: 51175067 and 51875086].

\section{Authors' contributions}

Shuai Fan and Shouwen Fan were in charge of the whole trial; Shuai Fan wrote the manuscript; Xin Zhang, Guangkui Song and Weibin Lan manufactured the prototype. All authors read and approved the final manuscript

\section{Acknowledgement}

Not applicable

Appendix A: The expressions of some matrices of 3-DOF system

$$
\begin{aligned}
& \boldsymbol{R}=\left[\begin{array}{ccc}
\mathrm{c} \beta & 0 & \mathrm{~s} \beta \\
\mathrm{s} \alpha \mathrm{s} \beta & \mathrm{c} \alpha & -\mathrm{s} \alpha \mathrm{c} \beta \\
-\mathrm{c} \alpha \mathrm{s} \beta & \mathrm{s} \alpha & \mathrm{c} \alpha \mathrm{c} \beta
\end{array}\right] \\
& \boldsymbol{J}_{\omega i}^{\prime}=-\frac{l_{i}^{\prime}}{l_{i}} \boldsymbol{J}_{\omega i}+\frac{1}{l_{i}}\left[\left(\boldsymbol{\omega}_{i} \times \boldsymbol{l}_{i}\right) \times \boldsymbol{R}_{\alpha}^{\prime} \boldsymbol{b}_{i 0}+\boldsymbol{l}_{i} \times\left(\boldsymbol{R}_{\alpha}^{\prime}\right)^{\prime} \boldsymbol{b}_{i \mathbf{0}} \quad\left(\boldsymbol{\omega}_{i} \times \boldsymbol{l}_{i}\right) \times \boldsymbol{R}_{\beta}^{\prime} \boldsymbol{b}_{i \mathbf{0}}+\boldsymbol{l}_{i} \times\left(\boldsymbol{R}_{\beta}^{\prime}\right)^{\prime} \boldsymbol{b}_{i \mathbf{0}} \quad\left(\boldsymbol{\omega}_{i} \times \boldsymbol{l}_{i}\right) \times \boldsymbol{l}_{\mathbf{4}}\right] \quad(i=1,2,3), \\
& \left(\boldsymbol{R}_{\alpha}^{\prime}\right)^{\prime}=\frac{\partial \boldsymbol{R}_{\alpha}^{\prime}}{\partial \alpha} \alpha^{\prime}+\frac{\partial \boldsymbol{R}_{\alpha}^{\prime}}{\partial \beta} \beta^{\prime}=\boldsymbol{R}_{\alpha \alpha}^{\prime \prime} \alpha^{\prime}+\boldsymbol{R}_{\alpha \beta}^{\prime \prime} \beta^{\prime}, \\
& \left(\boldsymbol{R}_{\beta}^{\prime}\right)^{\prime}=\frac{\partial \boldsymbol{R}_{\beta}^{\prime}}{\partial \alpha} \alpha^{\prime}+\frac{\partial \boldsymbol{R}_{\beta}^{\prime}}{\partial \beta} \beta^{\prime}=\boldsymbol{R}_{\beta \alpha}^{\prime \prime} \alpha^{\prime}+\boldsymbol{R}_{\beta \beta}^{\prime \prime} \beta^{\prime}, \\
& \boldsymbol{J}_{i \mathbf{1}}^{\prime}=-\frac{l_{i}^{\prime}}{l_{i}} \boldsymbol{J}_{i \mathbf{1}}+\left[\begin{array}{c}
\left(\left(\boldsymbol{\omega}_{i} \times \boldsymbol{l}_{i}\right) \boldsymbol{l}_{i}^{T}+\boldsymbol{l}_{i}\left(\boldsymbol{\omega}_{i} \times \boldsymbol{l}_{i}\right)^{T}\right) \boldsymbol{R}_{\alpha}^{\prime} \boldsymbol{b}_{i \mathbf{0}}+\left(\boldsymbol{E}_{\mathbf{3} \times \mathbf{3}}-\boldsymbol{l}_{i} \boldsymbol{l}_{i}^{T}\right)\left(\boldsymbol{R}_{\alpha}^{\prime}\right)^{\prime} \boldsymbol{b}_{i \mathbf{0}} \\
\left(\left(\boldsymbol{\omega}_{i} \times \boldsymbol{l}_{i}\right) \boldsymbol{l}_{i}^{T}+\boldsymbol{l}_{i}\left(\boldsymbol{\omega}_{i} \times \boldsymbol{l}_{i}\right)^{T}\right) \boldsymbol{R}_{\beta}^{\prime} \boldsymbol{b}_{i \mathbf{0}}+\left(\boldsymbol{E}_{\mathbf{3} \times \mathbf{3}}-\boldsymbol{l}_{i} \boldsymbol{l}_{i}^{T}\right)\left(\boldsymbol{R}_{\beta}^{\prime}\right)^{\prime} \boldsymbol{b}_{i \mathbf{0}} \\
\left(\left(\boldsymbol{\omega}_{i} \times \boldsymbol{l}_{i}\right) \boldsymbol{l}_{i}^{T}+\boldsymbol{l}_{i}\left(\boldsymbol{\omega}_{i} \times \boldsymbol{l}_{i}\right)^{T}\right) \boldsymbol{l}_{\mathbf{4}}
\end{array}\right]_{(i=1,2,3),} \\
& +\frac{l_{\mathbf{0}}}{\boldsymbol{2}_{i}}\left[\begin{array}{c}
\left(\left(\boldsymbol{\omega}_{i} \times \boldsymbol{l}_{i}\right) \boldsymbol{l}_{i}^{T}+\boldsymbol{l}_{i}\left(\boldsymbol{\omega}_{i} \times \boldsymbol{l}_{i}\right)^{T}\right) \boldsymbol{R}_{\alpha}^{\prime} \boldsymbol{b}_{i \mathbf{0}} \\
\left(\left(\boldsymbol{\omega}_{i} \times \boldsymbol{l}_{i}\right) \boldsymbol{l}_{i}^{T}+\boldsymbol{l}_{i}\left(\boldsymbol{\omega}_{i} \times \boldsymbol{l}_{i}\right)^{T}\right) \boldsymbol{R}_{\beta}^{\prime} \boldsymbol{b}_{i 0} \\
\left(\left(\omega_{i} \times \boldsymbol{l}_{i}\right) \boldsymbol{l}_{i}^{T}+\boldsymbol{l}_{i}\left(\boldsymbol{\omega}_{i} \times \boldsymbol{l}_{i}\right)^{T}\right) \boldsymbol{l}_{\mathbf{4}}
\end{array}\right]^{T} \\
& \boldsymbol{J}_{i \mathbf{2}}^{\prime}=\frac{l_{i}^{\prime}}{l_{i \mathbf{0}}} \boldsymbol{J}_{i \mathbf{1}}+\frac{l_{i \mathbf{0}}+l_{i}}{l_{i \mathbf{0}}} \boldsymbol{J}_{i \mathbf{1}}^{\prime}+\frac{\mathbf{1}}{\mathbf{2}}\left[\begin{array}{c}
\left(\left(\boldsymbol{\omega}_{i} \times \boldsymbol{l}_{i}\right) \boldsymbol{l}_{i}^{T}+\boldsymbol{l}_{i}\left(\boldsymbol{\omega}_{i} \times \boldsymbol{l}_{i}\right)^{T}\right) \boldsymbol{R}_{\alpha}^{\prime} \boldsymbol{b}_{i \mathbf{0}}+\boldsymbol{l}_{i} \boldsymbol{l}_{i}^{T}\left(\boldsymbol{R}_{\alpha}^{\prime}\right)^{\prime} \boldsymbol{b}_{i \mathbf{0}} \\
\left.\boldsymbol{\omega}_{i} \times \boldsymbol{l}_{i}^{T}+\boldsymbol{l}_{i}\left(\omega_{i} \times \boldsymbol{l}_{i}\right)^{T}\right) \boldsymbol{R}_{\beta}^{\prime} \boldsymbol{b}_{i \mathbf{0}}+\boldsymbol{l}_{i} \boldsymbol{l}_{i}^{T}\left(\boldsymbol{R}_{\beta}^{\prime}\right)^{\prime} \boldsymbol{b}_{i \mathbf{0}} \\
\left(\left(\boldsymbol{\omega}_{i} \times \boldsymbol{l}_{i}\right) \boldsymbol{l}_{i}^{T}+\boldsymbol{l}_{i}\left(\boldsymbol{\omega}_{i} \times \boldsymbol{l}_{i}\right)^{T}\right) \boldsymbol{l}_{\mathbf{4}}
\end{array}\right]^{T} \quad(i=1,2,3) .
\end{aligned}
$$

Appendix B: the expressions of some matrices of 6-DOF system

$$
\boldsymbol{R}=\left[\begin{array}{ccc}
\mathrm{c}(\beta+\varphi) \mathrm{c} \psi & -\mathrm{c}(\beta+\varphi) \mathrm{s} \psi & \mathrm{s}(\beta+\varphi) \\
\mathrm{c}(\alpha+\theta) \mathrm{s} \psi+\mathrm{s}(\alpha+\theta) \mathrm{s}(\beta+\varphi) \mathrm{c} \psi & \mathrm{c}(\alpha+\theta) \mathrm{c} \psi-\mathrm{s}(\alpha+\theta) \mathrm{s}(\beta+\varphi) \mathrm{s} \psi & -\mathrm{s}(\alpha+\theta) \mathrm{c}(\beta+\varphi) \\
\mathrm{s}(\alpha+\theta) \mathrm{s} \psi-\mathrm{c}(\alpha+\theta) \mathrm{s}(\beta+\varphi) \mathrm{c} \psi & \mathrm{s}(\alpha+\theta) \mathrm{c} \psi+\mathrm{c}(\alpha+\theta) \mathrm{s}(\beta+\varphi) \mathrm{s} \psi & \mathrm{c}(\alpha+\theta) \mathrm{c}(\beta+\varphi)
\end{array}\right]
$$




$$
\begin{aligned}
& \boldsymbol{J}_{\omega i}^{\prime}=-\frac{l_{i}^{\prime}}{l_{i}} \boldsymbol{J}_{\omega i}+\frac{\mathbf{1}}{l_{i}}\left[\begin{array}{c}
\left(\boldsymbol{\omega}_{i} \times \boldsymbol{l}_{i}\right) \times \boldsymbol{R}_{\theta}^{\prime} \boldsymbol{b}_{i 0}+\boldsymbol{l}_{i} \times\left(\boldsymbol{R}_{\theta}^{\prime}\right)^{\prime} \boldsymbol{b}_{i \mathbf{0}} \\
\left(\boldsymbol{\omega}_{i} \times \boldsymbol{l}_{i}\right) \times \boldsymbol{R}_{\varphi}^{\prime} \boldsymbol{b}_{i 0}+\boldsymbol{l}_{i} \times\left(\boldsymbol{R}_{\varphi}^{\prime}\right)^{\prime} \boldsymbol{b}_{i \mathbf{0}} \\
\left(\boldsymbol{\omega}_{i} \times \boldsymbol{l}_{i}\right) \times \boldsymbol{R}_{\psi}^{\prime} \boldsymbol{b}_{i \mathbf{0}}+\boldsymbol{l}_{i} \times\left(\boldsymbol{R}_{\psi}^{\prime}\right)^{\prime} \boldsymbol{b}_{i \mathbf{0}} \\
\left(\boldsymbol{\omega}_{i} \times \boldsymbol{l}_{i}\right) \times \boldsymbol{n}_{x} \\
\left(\boldsymbol{\omega}_{i} \times \boldsymbol{l}_{i}\right) \times \boldsymbol{n}_{y} \\
\left(\boldsymbol{\omega}_{i} \times \boldsymbol{l}_{i}\right) \times \boldsymbol{n}_{z}
\end{array}\right]_{(i=1,2,3)} \\
& \left(\boldsymbol{R}_{\theta}^{\prime}\right)^{\prime}=\frac{\partial \boldsymbol{R}_{\theta}^{\prime}}{\partial \theta} \theta^{\prime}+\frac{\partial \boldsymbol{R}_{\theta}^{\prime}}{\partial \varphi} \varphi^{\prime}+\frac{\partial \boldsymbol{R}_{\theta}^{\prime}}{\partial \psi} \psi^{\prime}, \\
& \left(\boldsymbol{R}_{\varphi}^{\prime}\right)^{\prime}=\frac{\partial \boldsymbol{R}_{\varphi}^{\prime}}{\partial \theta} \theta^{\prime}+\frac{\partial \boldsymbol{R}_{\varphi}^{\prime}}{\partial \varphi} \varphi^{\prime}+\frac{\partial \boldsymbol{R}_{\varphi}^{\prime}}{\partial \psi} \psi^{\prime}, \\
& \left(\boldsymbol{R}_{\psi}^{\prime}\right)^{\prime}=\frac{\partial \boldsymbol{R}_{\psi}^{\prime}}{\partial \theta} \theta^{\prime}+\frac{\partial \boldsymbol{R}_{\psi}^{\prime}}{\partial \varphi} \varphi^{\prime}+\frac{\partial \boldsymbol{R}_{\psi}^{\prime}}{\partial \psi} \psi^{\prime}, \\
& \boldsymbol{J}_{i \mathbf{1}}^{\prime}=-\frac{l_{i}^{\prime}}{l_{i}} \boldsymbol{J}_{i \mathbf{1}}+\frac{l_{i \mathbf{0}}}{\boldsymbol{2} l_{i}}\left[\begin{array}{c}
\left(\boldsymbol{E}-\boldsymbol{l}_{i} \boldsymbol{l}_{i}^{T}\right)\left(\boldsymbol{R}_{\theta}^{\prime}\right)^{\prime} \boldsymbol{b}_{i 0}-\left(\left(\boldsymbol{\omega}_{i} \times \boldsymbol{l}_{i}\right) \boldsymbol{l}_{i}^{T}+\boldsymbol{l}_{i} \times\left(\boldsymbol{\omega}_{i} \times \boldsymbol{l}_{i}\right)^{T}\right) \boldsymbol{R}_{\theta}^{\prime} \boldsymbol{b}_{i \mathbf{0}} \\
\left(\boldsymbol{E}-\boldsymbol{l}_{i} \boldsymbol{l}_{i}^{T}\right)\left(\boldsymbol{R}_{\varphi}^{\prime}\right)^{\prime} \boldsymbol{b}_{i 0}-\left(\left(\boldsymbol{\omega}_{i} \times \boldsymbol{l}_{i}\right) \boldsymbol{l}_{i}^{T}+\boldsymbol{l}_{i} \times\left(\boldsymbol{\omega}_{i} \times \boldsymbol{l}_{i}\right)^{T}\right) \boldsymbol{R}_{\varphi}^{\prime} \boldsymbol{b}_{i 0} \\
-\left(\left(\boldsymbol{R}_{i}^{\prime}\right)^{\prime} \boldsymbol{b}_{i 0}-\left(\left(\boldsymbol{l}_{i} \times \boldsymbol{l}_{i}\right) \boldsymbol{l}_{i}^{T}+\boldsymbol{l}_{i} \times\left(\boldsymbol{l}_{i} \times\left(\boldsymbol{l}_{i} \times\right)^{T}\right) \boldsymbol{R}_{i}^{\prime}\right)^{T}\right) \boldsymbol{n}_{x} \\
-\left(\left(\boldsymbol{\omega}_{i} \times \boldsymbol{l}_{i}\right) \boldsymbol{l}_{i}^{T}+\boldsymbol{l}_{i} \times\left(\boldsymbol{\omega}_{i} \times \boldsymbol{l}_{i}\right)^{T}\right) \boldsymbol{n}_{x} \boldsymbol{n}_{y} \\
-\left(\left(\boldsymbol{\omega}_{i} \times \boldsymbol{l}_{i}\right) \boldsymbol{l}_{i}^{T}+\boldsymbol{l}_{i} \times\left(\boldsymbol{\omega}_{i} \times \boldsymbol{l}_{i}\right)^{T}\right) \boldsymbol{n}_{x} \boldsymbol{n}_{z}
\end{array}\right]^{T}
\end{aligned}
$$

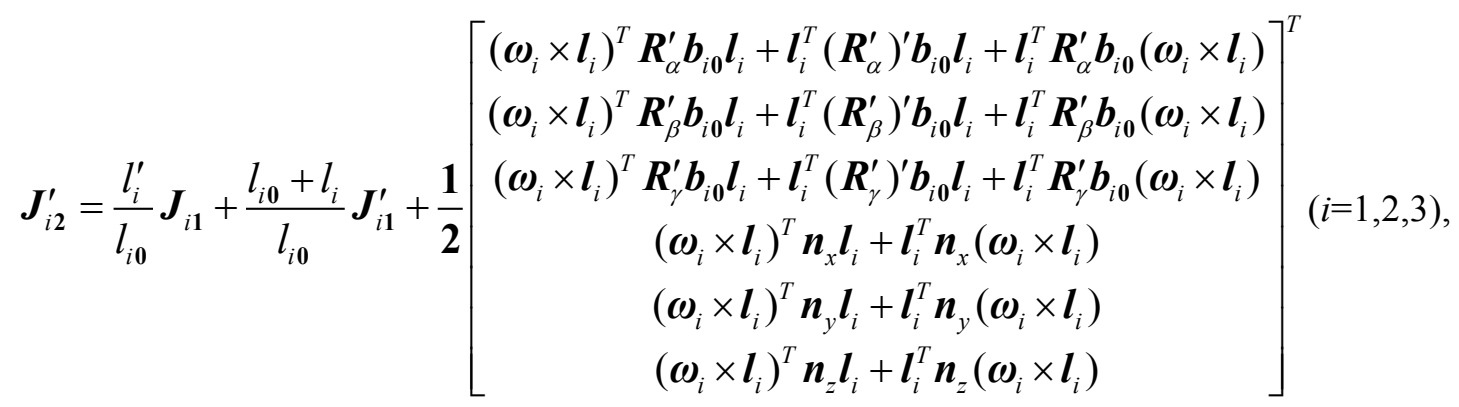

$$
\begin{aligned}
& \boldsymbol{J}_{41}^{\prime}=\boldsymbol{J}_{42}^{\prime}=-\frac{l_{4}^{\prime}}{l_{4}} \boldsymbol{J}_{41}+\frac{l_{40}}{2 l_{4}}\left[\begin{array}{c}
0 \\
0 \\
0 \\
-\frac{o_{x}^{\prime}}{l_{4}^{2}} \boldsymbol{o}+\frac{\mathbf{2} o_{x}}{l_{4}^{3}} l_{4}^{\prime} \boldsymbol{o}-\frac{o_{x}}{l_{4}^{2}} \boldsymbol{o}^{\prime} \\
-\frac{o_{y}^{\prime}}{l_{4}^{2}} \boldsymbol{o}+\frac{\mathbf{2}_{y}}{l_{4}^{3}} l_{4}^{\prime} \boldsymbol{o}-\frac{o_{y}}{l_{4}^{2}} \boldsymbol{o}^{\prime} \\
-\frac{o_{z}^{\prime}}{l_{4}^{2}} \boldsymbol{o}+\frac{2 o_{z}}{l_{4}^{3}} l_{4}^{\prime} \boldsymbol{o}-\frac{o_{z}}{l_{4}^{2}} \boldsymbol{o}^{\prime}
\end{array}\right]^{T}
\end{aligned}
$$




\section{References}

[1] B. Stone, Chatter and machine tool, Springer International Publishing Switzerland 2014.

[2] W.F. S.A. Tobias, The vibrations of radial-drlling machines under test and working conditions, Proc Inst Mech Eng 170 (1956) 232-247.

[3] P. Kumar, B. Pratiher, Nonlinear modeling and vibration analysis of a two-link flexible manipulator coupled with harmonically driven flexible joints, Mech. Mach. Theory, 131 (2019) 278-299.

[4] D. Kamesh, R. Pandiyan, A. Ghosal, Modeling, design and analysis of low frequency platform for attenuating micro-vibration in spacecraft, J. Sound Vibr., 329 (2010) 3431-3450.

[5] M. Mahboubkhah, M.J. Nategh, S.E. Khadem, Vibration analysis of machine tool's hexapod table, Int. J. Adv. Manuf. Technol., 38 (2008) 1236-1243.

[6] R. Gexue, L. Qiuhai, H. Ning, N. Rendong, P. Bo, On vibration control with Stewart parallel mechanism, Mechatronics, 14 (2004) 1-13.

[7] Y. Wu, K.P. Yu, J. Jiao, R. Zhao, Dynamic modeling and robust nonlinear control of a six-DOF active microvibration isolation manipulator with parameter uncertainties, Mech. Mach. Theory, 92 (2015) 407-435.

[8] D. Hong, S. Kim, W.C. Choi, J.B. Song, Analysis of machining stability for a parallel machine tool, Mech. Based Des. Struct. Mech., 31 (2003) 509-528.

[9] P. Mukherjee, B. Dasgupta, A.K. Mallik, Dynamic stability index and vibration analysis of a flexible Stewart platform, J. Sound Vibr., 307 (2007) 495-512.

[10] X.L. Yang, H.T. Wu, Y. Li, B. Chen, Dynamic isotropic design and decentralized active control of a six-axis vibration isolator via Stewart platform, Mech. Mach. Theory, 117 (2017) 244-252.

[11] B. Afzali-Far, A. Andersson, K. Nilsson, P. Lidstrom, Influence of strut inertia on the vibrations in initially symmetric Gough-Stewart Platforms-an analytical study, J. Sound Vibr., 352 (2015) 142-157.

[12] Y. Wu, K.P. Yu, J. Jiao, D.Q. Cao, W.C. Chi, J. Tang, Dynamic isotropy design and analysis of a six-DOF active micro-vibration isolation manipulator on satellites, Robotics and Computer-Integrated Manufacturing, 49 (2018) 408-425.

[13] S. Pedrammehr, M. Mahboubkhah, N. Khani, A study on vibration of Stewart platform-based machine tool table, Int. J. Adv. Manuf. Technol., 65 (2013) 991-1007.

[14] X.L. Yang, H.T. Wu, B. Chen, S.Z. Kang, S.L. Cheng, Dynamic modeling and decoupled control of a flexible Stewart platform for vibration isolation, J. Sound Vibr., 439 (2019) 398-412.

[15] Z.Z. Chi, D. Zhang, L. Xia, Z. Gao, Multi-objective optimization of stiffness and workspace for a parallel kinematic machine, Int. J. Mech. Mater. Des., 9 (2013) 281-293.

[16] D. Wang, J. Wu, L.P. Wang, Y.Z. Liu, A Postprocessing Strategy of a 3-DOF Parallel Tool Head Based on Velocity Control and Coarse Interpolation, IEEE Trans. Ind. Electron., 65 (2018) 6333-6342.

[17] C.L. Dong, H.T. Liu, W. Yue, T. Huang, Stiffness modeling and analysis of a novel 5-DOF hybrid robot, Mech. Mach. Theory, 125 (2018) 80-93.

[18] D. Chablat, P. Wenger, Architecture optimization of a 3-DOF translational parallel mechanism for machining applications, the Orthoglide, IEEE Trans. Robot. Autom., 19 (2003) 403-410.

[19] D. Chablat, E. Ottaviano, S. Venkateswaran, Self-motion conditions for a 3-(PP)under-barPS parallel robot with delta-shaped base, Mech. Mach. Theory, 135 (2019) 109-114.

[20] K.M. Zheng, Q.J. Zhang, Comprehensive analysis of the position error and vibration characteristics of Delta robot, Adv. Robot., 30 (2016) 1322-1340.

[21] L.P. Wang, G. Yu, J. Wu, A comparison study on the stiffness and natural frequency of a redundant parallel conveyor and its nonredundant counterpart, Adv. Mech. Eng., 9 (2017) 11.

[22] L.C. Sheng, W. Li, Y.Q. Wang, M.B. Fan, X.F. Yang, Dynamic Model and Vibration Characteristics of Planar 3-RRR Parallel Manipulator with Flexible Intermediate Links considering Exact Boundary Conditions, Shock Vib., (2017) 13.

[23] K.F. Wen, C.B. Shin, T. Seo, J.W. Lee, Stiffness synthesis of 3-DOF planar 3RPR parallel mechanisms, Robotica, 34 (2016) 2776-2787.

[24] B. Li, W. Zhao, Z.Q. Deng, Modeling and analysis of a multi-dimensional vibration isolator based on the parallel mechanism, J. Manuf. Syst., 31 (2012) 50-58. 
[25] B. Li, W. Zhao, S. Wang, H.L. Huang, Dynamic modeling and control for a five-dimensional hybrid vibration isolator based on a position/orientation decoupled parallel mechanism, J. Vib. Control, 22 (2016) 3368-3383.

[26] N.S. Viliani, H. Zohoor, M.H. Kargarnovin, Vibration Analysis of a New Type of Compliant Mechanism with Flexible-Link, Using Perturbation Theory, Math. Probl. Eng., (2012) 19.

[27] X.L. Chen, L.K. Wu, Y. Deng, Q. Wang, Dynamic response analysis and chaos identification of 4-UPS-UPU flexible spatial parallel mechanism, Nonlinear Dyn., 87 (2017) 2311-2324.

[28] B.B. Lian, T. Sun, Y.M. Song, Y. Jin, M. Price, Stiffness analysis and experiment of a novel 5-DoF parallel kinematic machine considering gravitational effects, Int. J. Mach. Tools Manuf., 95 (2015) 82-96.

[29] G.L. Wu, Parameter Excited Instabilities of a 2UPU-RUR-RPS Spherical Parallel Manipulator With a Driven Universal Joint, J. Mech. Des., 140 (2018) 9.

[30] G.L. Wu, B. Niu, Dynamic stability of a tripod parallel robotic wrist featuring continuous end-effector rotation used for drill point grinder, Mech. Mach. Theory, 129 (2018) 36-50.

[31] M. Mahboubkhah, M.J. Nategh, S.E. Khadem, A comprehensive study on the free vibration of machine tools' hexapod table, Int. J. Adv. Manuf. Technol., 40 (2009) 1239-1251.

[32] T. Sun, B.B. Lian, Stiffness and mass optimization of parallel kinematic machine, Mech. Mach. Theory, 120 (2018) 73-88.

[33] S. Fan, S.W. Fan, Approximate Stiffness Modelling and Stiffness Defect Identification for a Heavy-load Parallel Manipulator, Robotica, 37 (2019) 1120-1142.

[34] Q. Tian, P. Flores, H.M. Lankarani, A comprehensive survey of the analytical, numerical and experimental methodologies for dynamics of multibody mechanical systems with clearance or imperfect joints, Mech. Mach. Theory, 122 (2018) 1-57.

[35] S. Fan, S.W. Fan, An improved approach to the inverse dynamic analysis of parallel manipulators by a given virtual screw, Adv. Robot., 32 (2018) 887-902.

[36] M. Pirtini, I. Lazoglu, Forces and hole quality in drilling, Int. J. Mach. Tools Manuf., 45 (2005) 1271-1281. 


\section{Figures}

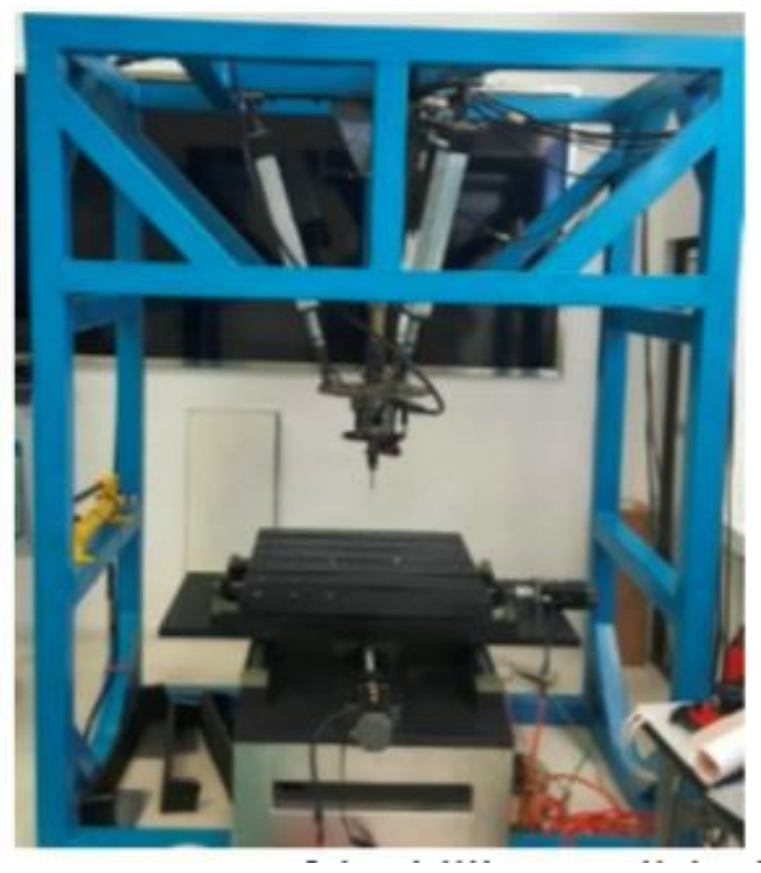

\section{Figure 1}

Prototype of the drilling parallel robot

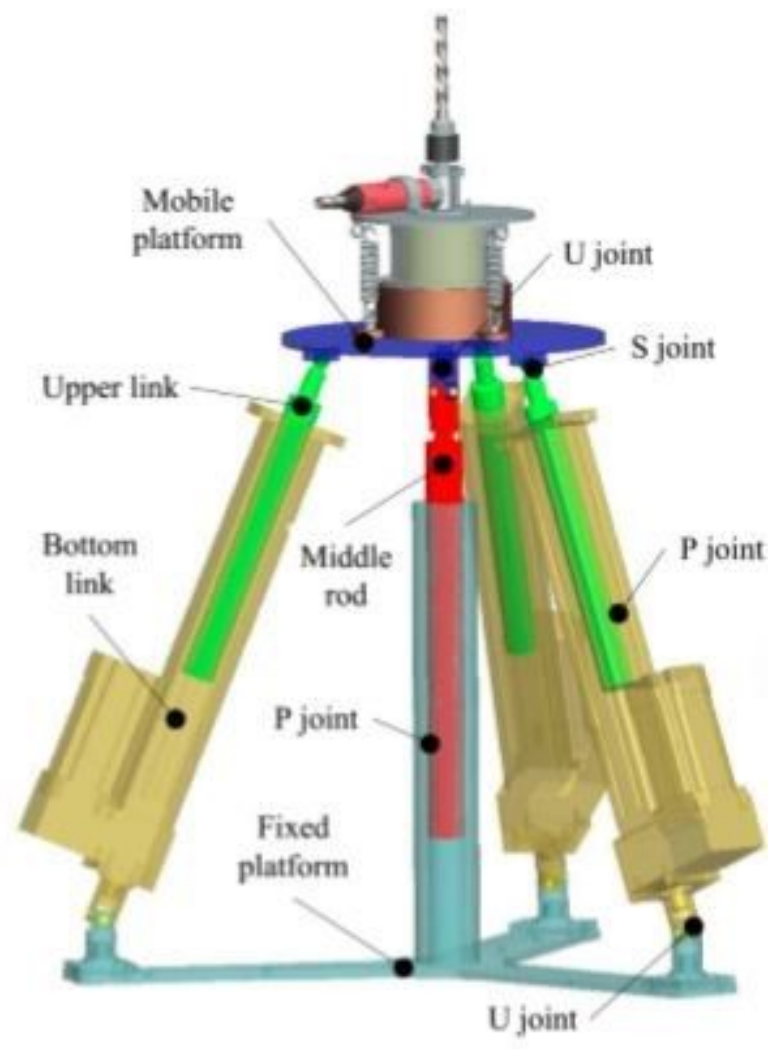

Figure 2 


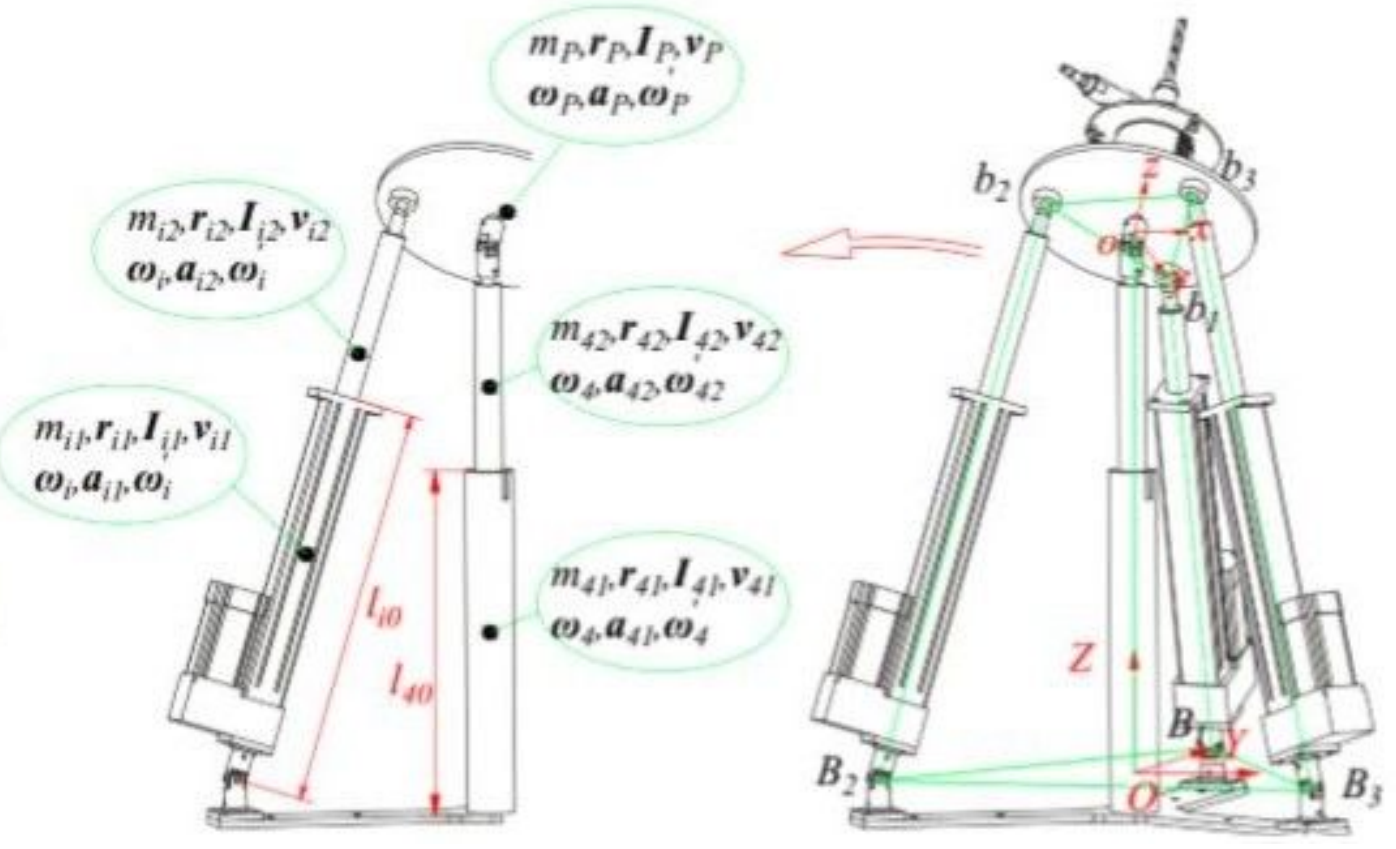

Figure 3

Parameter diagram of the drilling parallel robot

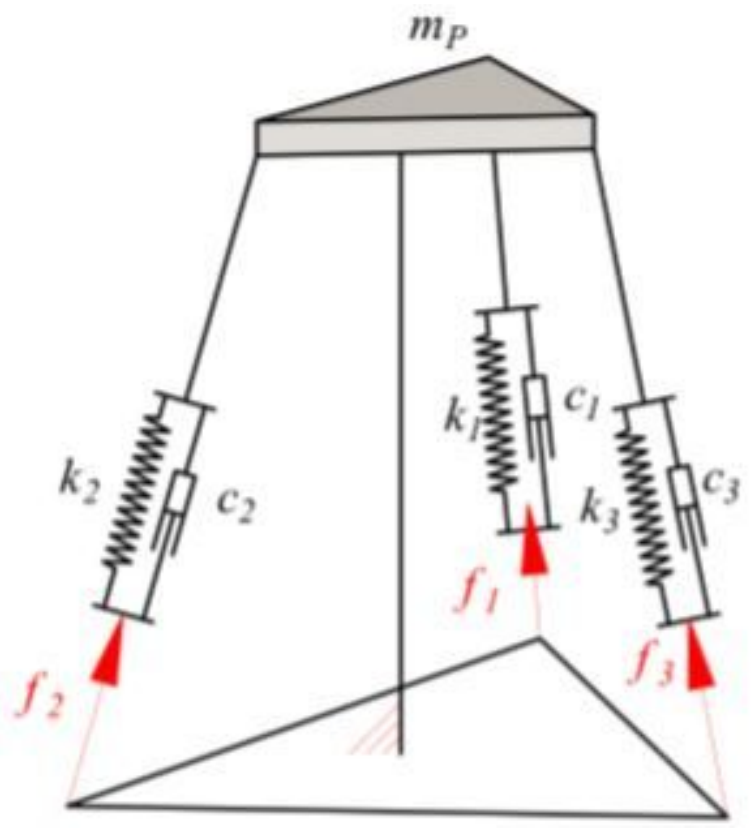

Figure 4

Structural diagram of the 3-DOF vibration system 

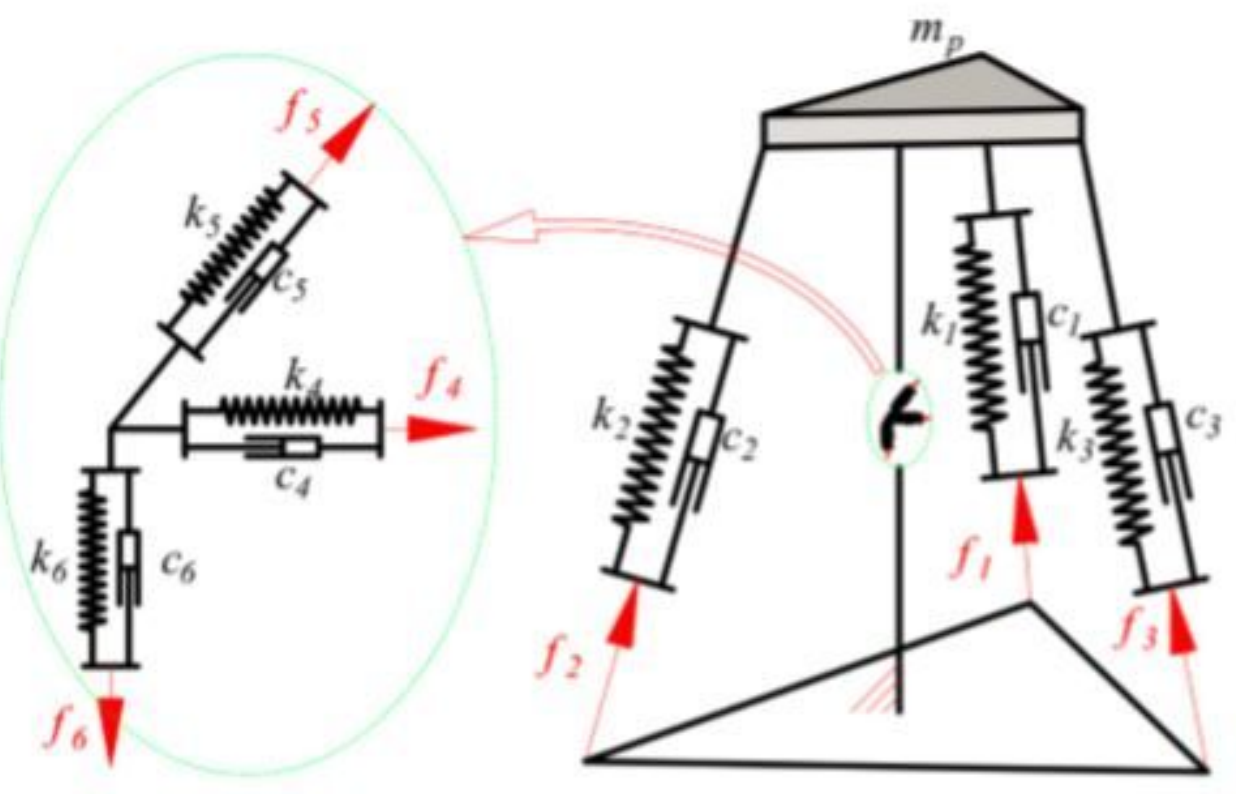

Figure 5

Structural diagram of the 6-DOF vibration system
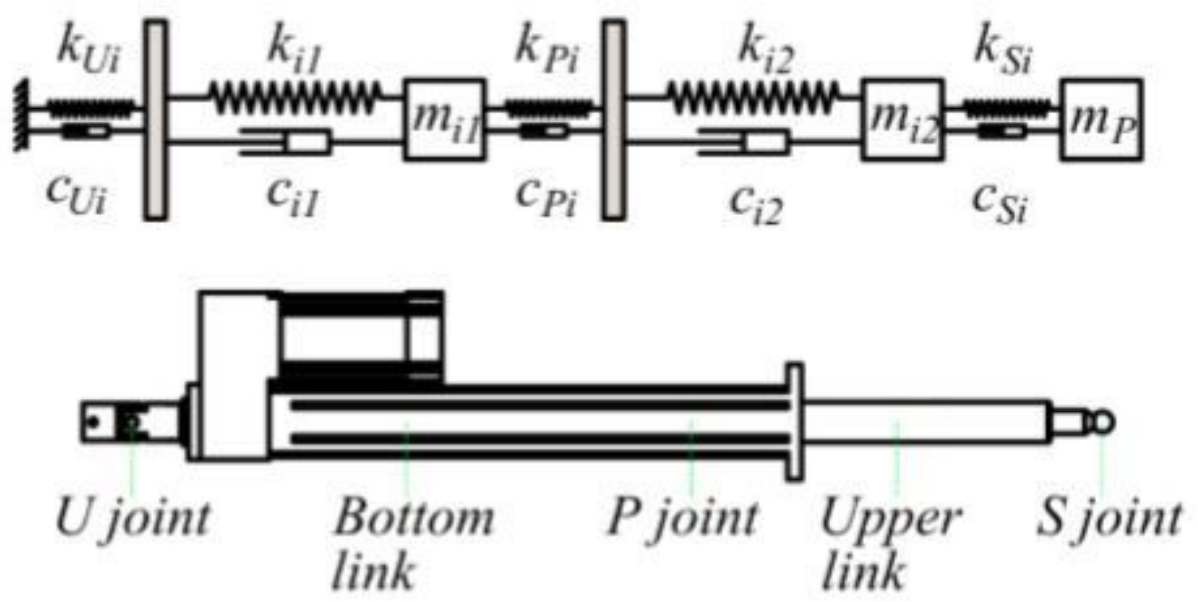

Figure 6

Stiffness model of the ith active branch 


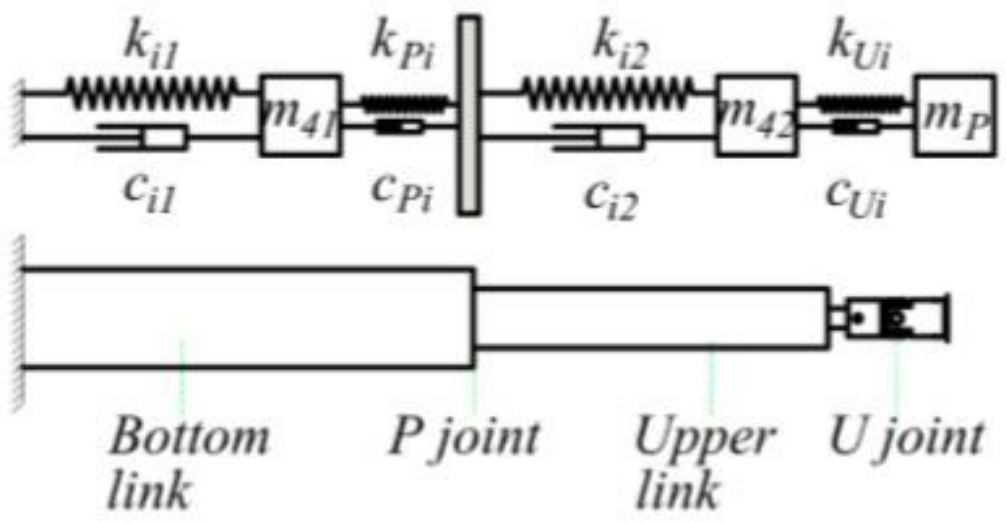

Figure 7

Stiffness model of the passive branch

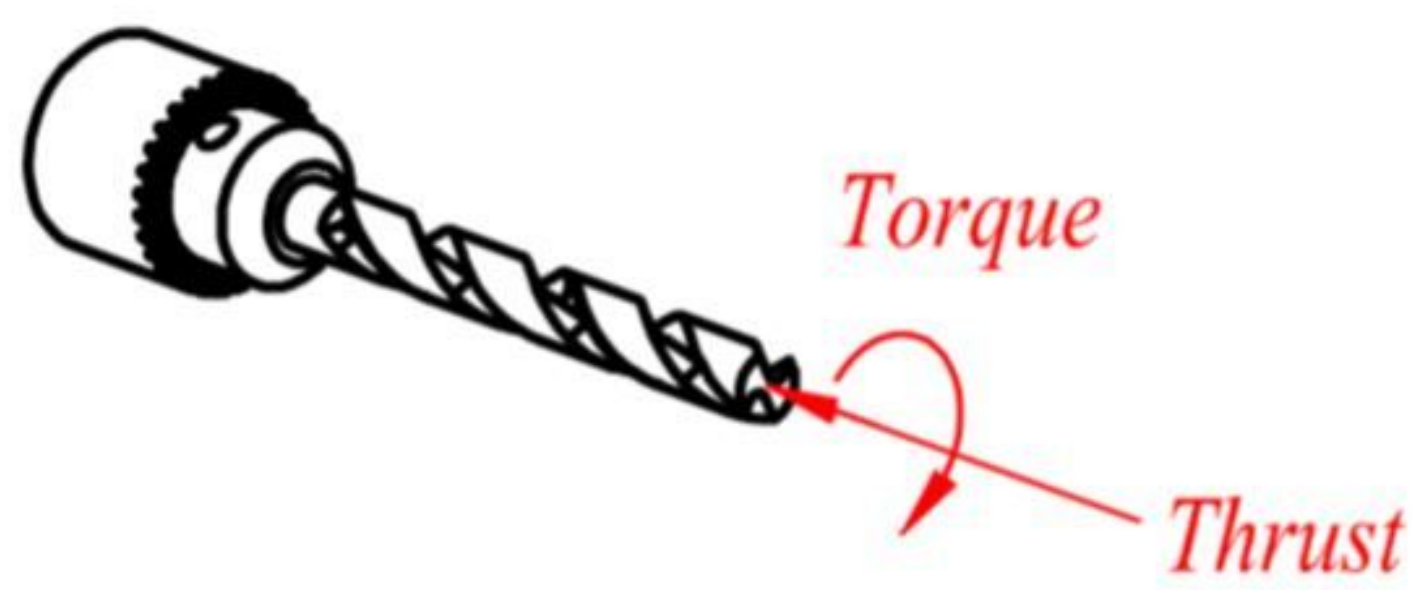

Figure 8

Mechanics of drilling Translating the drilling force into reference frame 0-XYZ yields the following expression for the external load: 
(a)

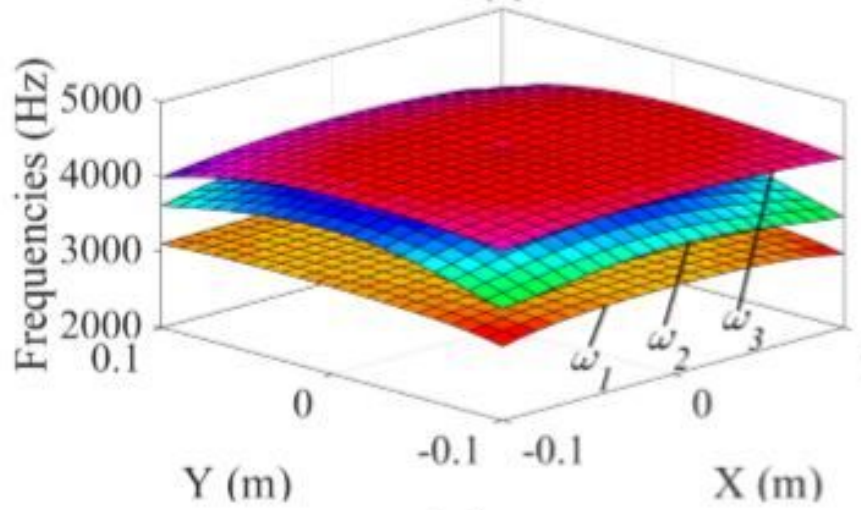

(c)

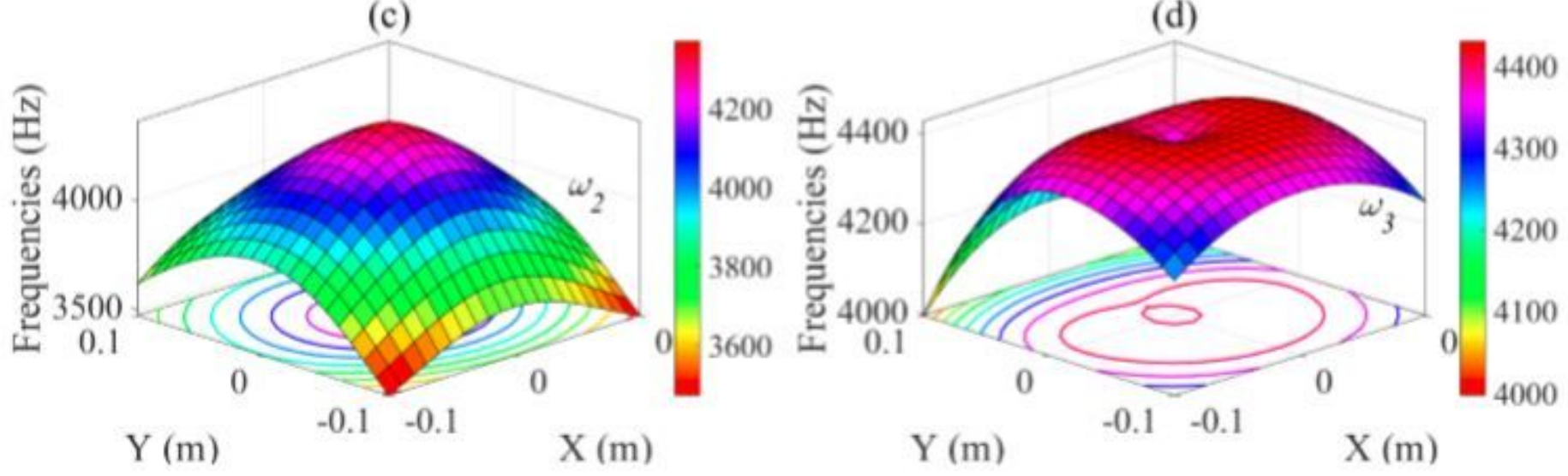

Figure 9

Natural frequencies of the 3-DOF vibration system: (a) The three ideal natural frequencies; (b) (d) the first through third natural frequencies. (b)

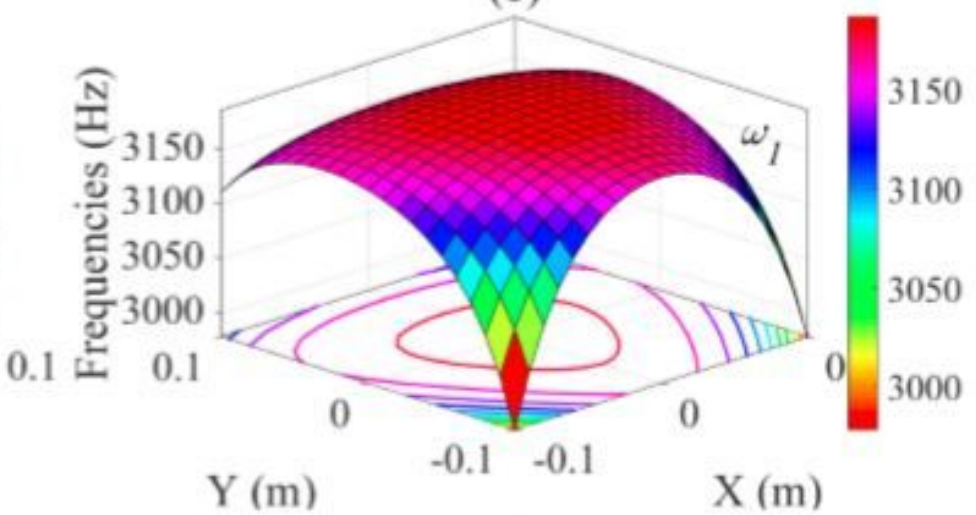

(d) 
(a)

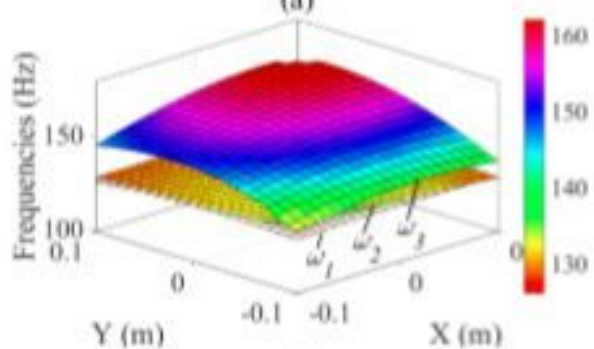

(c)

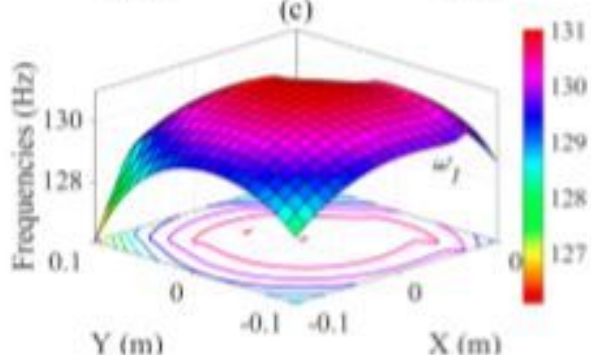

(e)

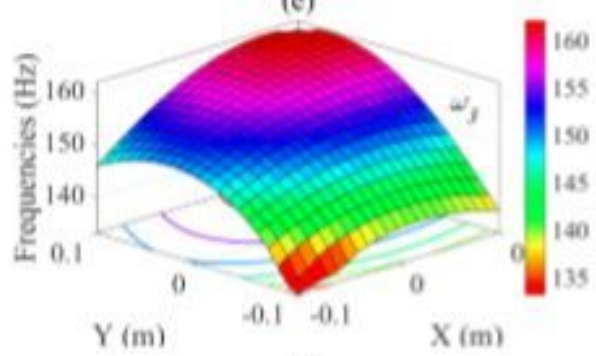

(g)

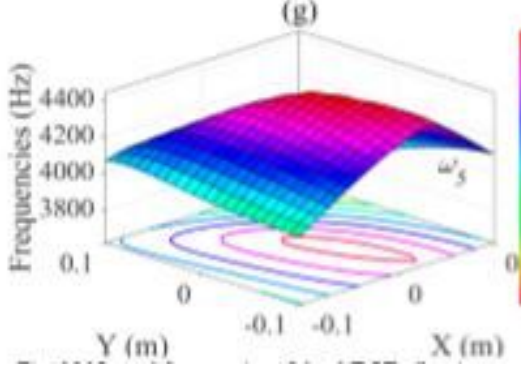

$Y(m)$

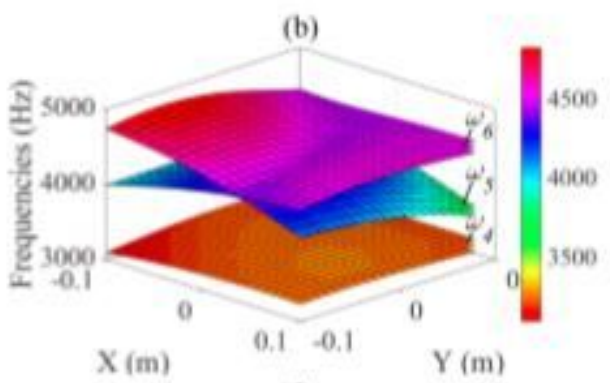

(d)

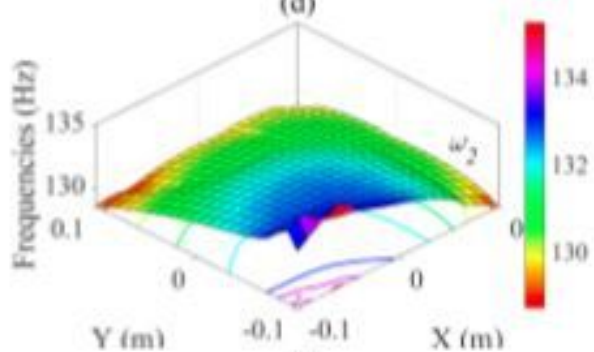

(f)

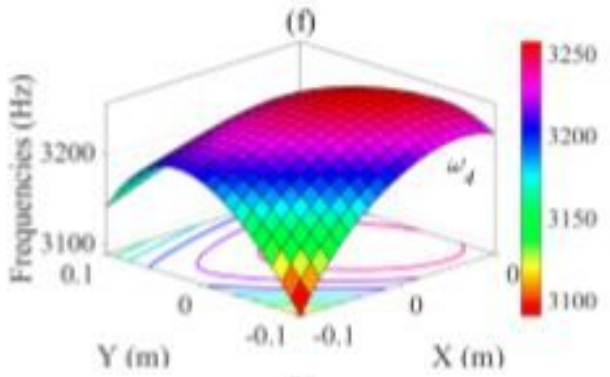

(b)

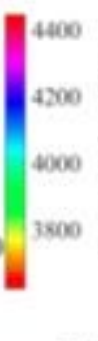

Figure 10

Natural frequencies of the 6-DOF vibration system: (a) The three ideal natural frequencies; (b) the three parasitic natural frequencies; and (c) (h) the first through sixth natural frequencies. in which the subscripts of the uppercase letters represent the elements in their corresponding matrices. Finally, substituting the parameter values into the vibration equation, the vibration amplitudes of the 3-DOF and 6-DOF

(a)

(b)

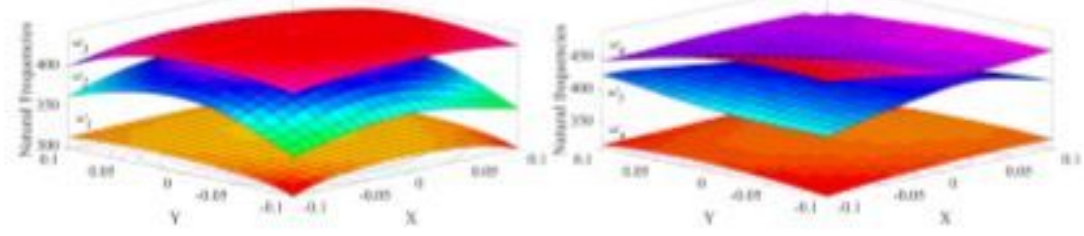

Figure 11 
The natural frequency of the system that changes the value of the driving stiffness: (a) 3-DOF system; (b) 6-DOF system vibration systems can be solved via $D=P$ Qu and are plotted in Fig. 12 and Fig. 13, respectively. For the 3-DOF vibration system, the vibrations of $\Delta a, \Delta \beta$, and $\Delta \mid 4$ are called the three original vibrations. For the 6-DOF vibration system, the vibrations of $\Delta \mathrm{a}, \Delta \beta$, and $\Delta \mathrm{Z}$ are called the three original vibrations and those of $\Delta \mathrm{X}, \Delta \mathrm{Y}$ and $\Delta \mathrm{Y}$ are called the three parasitic vibrations.
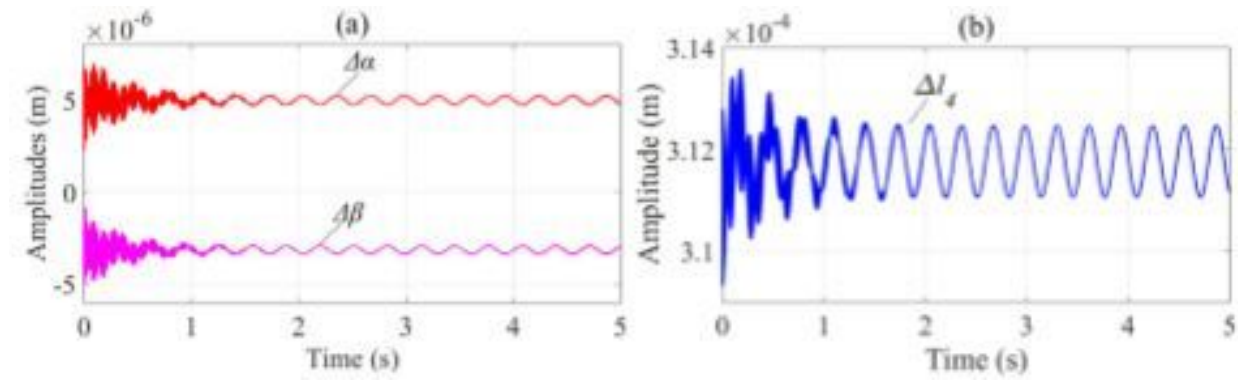

\section{Figure 12}

Forced vibration amplitudes of the 3-DOF vibration system: (a) two rotational vibrations; (b) one translational vibration.
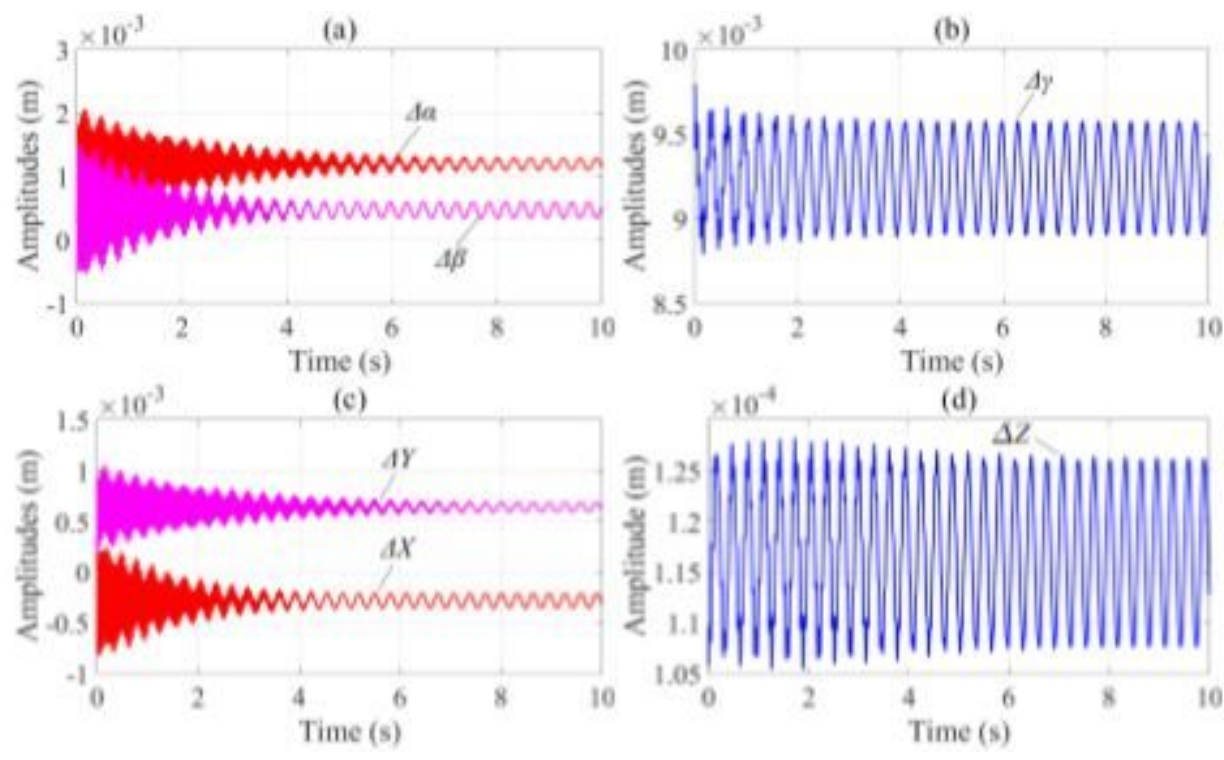

\section{Figure 13}

Forced vibration amplitudes of the 6-DOF vibration system: (a) rotational vibrations around the $\mathrm{X}$-axis and the $\mathrm{Y}$-axis; (b) rotational vibration around the Z-axis; (c) translational vibrations along the $\mathrm{X}$-axis and the Y-axis; (d) translational vibration along the Z-axis.

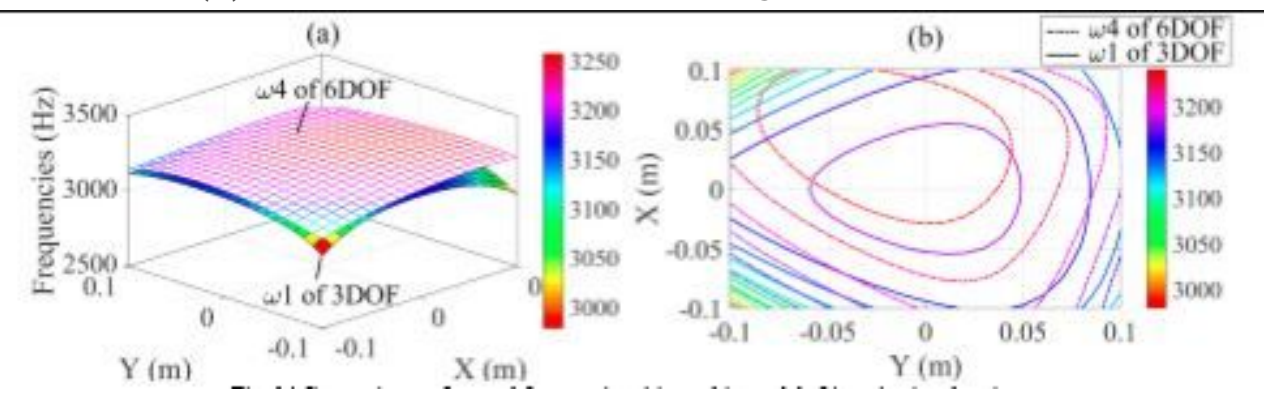


Figure 14

Comparisons of natural frequencies: (a) graphic model; (b) projection drawing.
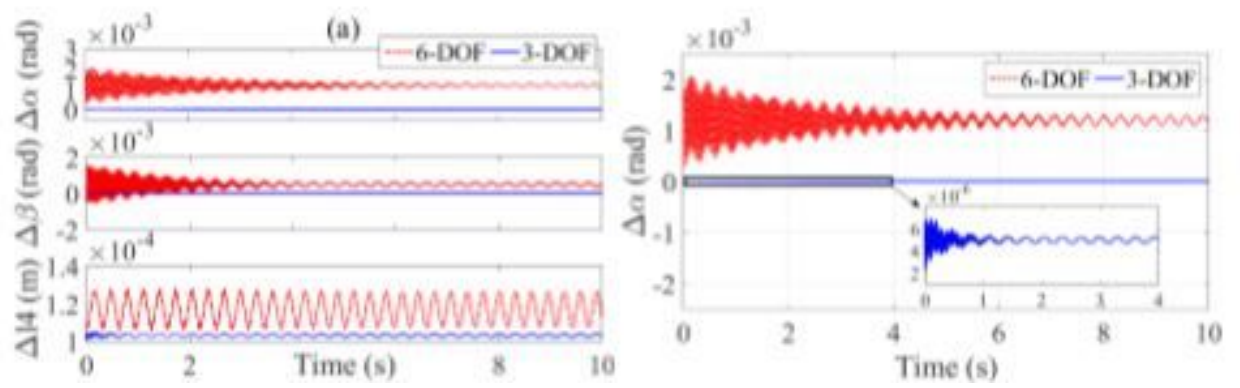

\section{Figure 15}

Vibration behaviour comparisons between two vibration systems: (a) three original motions; (b) around the $\mathrm{X}$-axis.
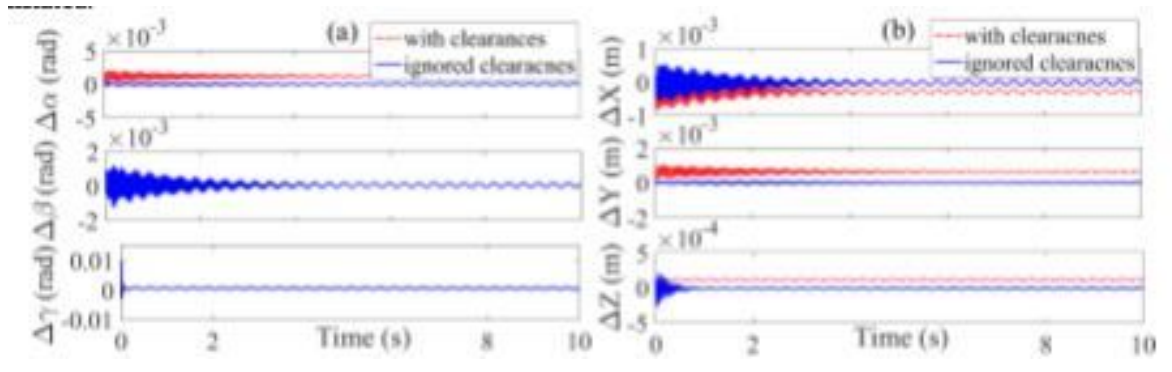

\section{Figure 16}

Effects of the joint clearances on the 6-DOF vibration system: (a) rotational vibrations; (b) translational vibrations.

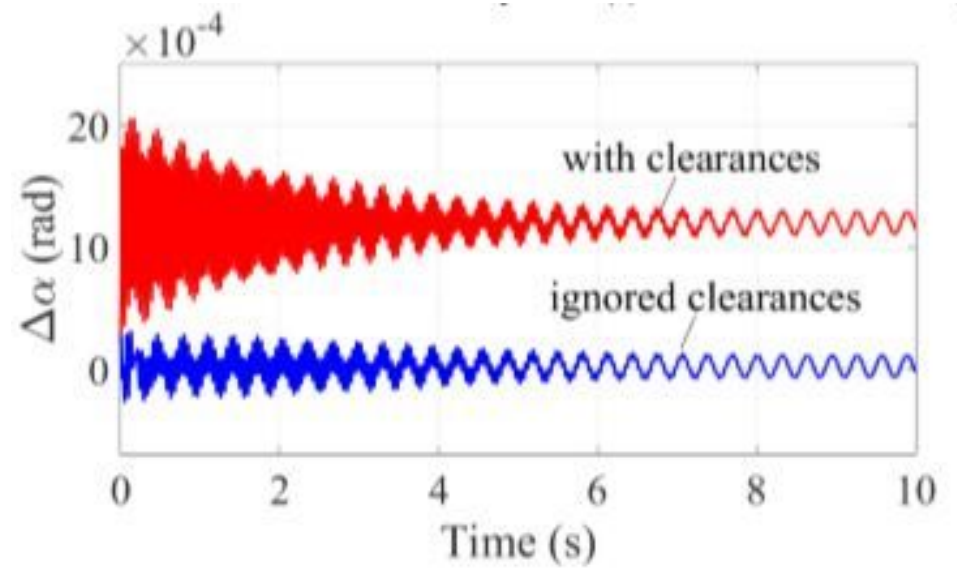

Figure 17

Effects of the joint clearances around the X-axis direction 

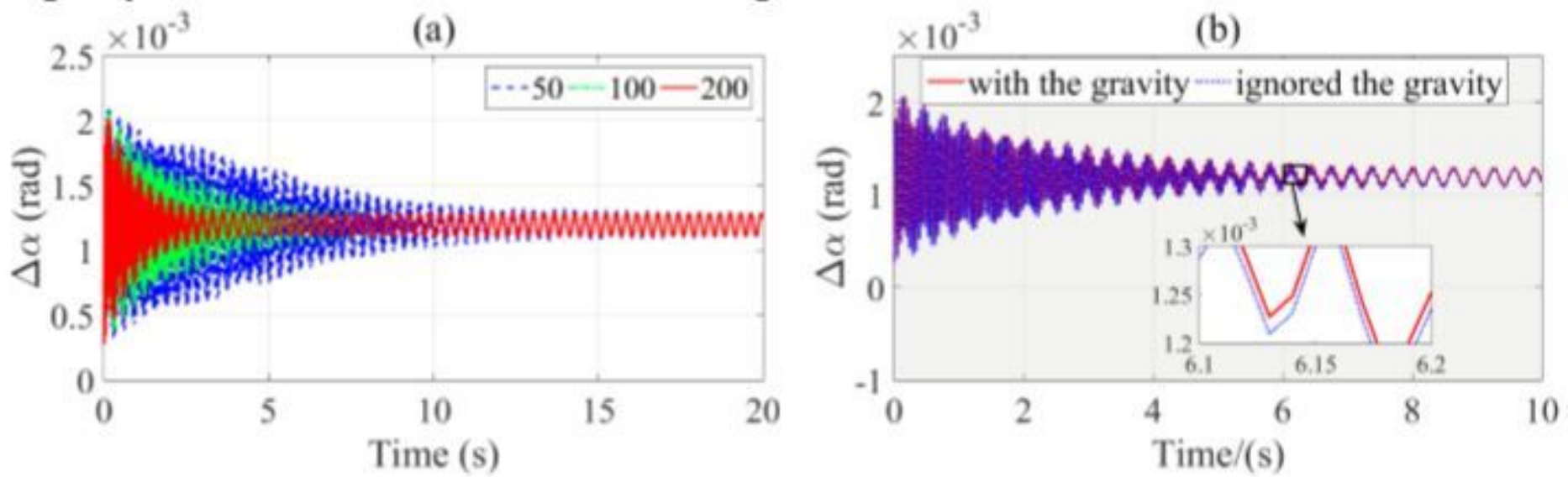

\section{Figure 18}

Effects of damping and gravity: (a) damping; (b) gravity.

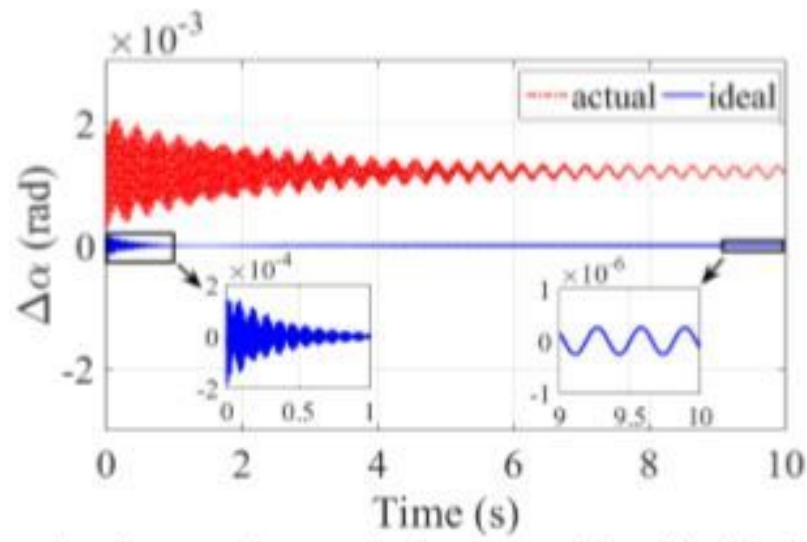

\section{Figure 19}

Comparison between the actual vibration model and the ideal vibration model

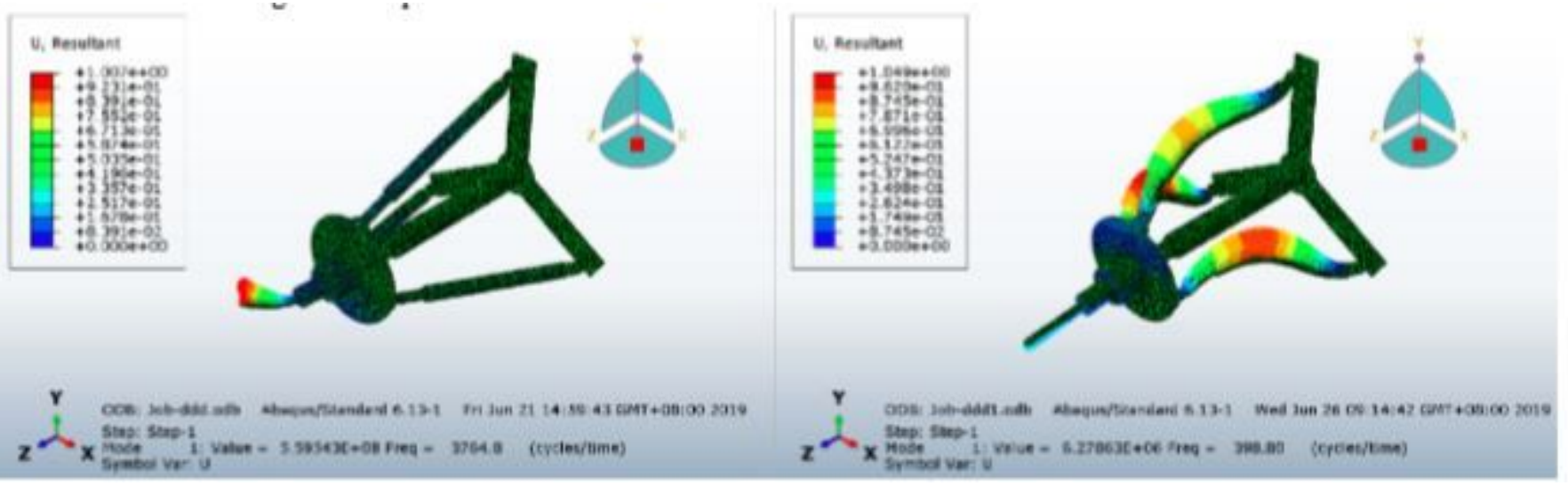

Figure 20

The first natural frequencies solved by ABAQUS: (a) the 3-DOF vibration system; (b) the 6-DOF vibration system. 